Florida International University

FIU Digital Commons

FIU Electronic Theses and Dissertations

University Graduate School

3-30-2016

\title{
Physical and Chemical Characterization of Crude Oil-Water Mixtures: Understanding the Effects of Interfacial Process to Chemical Bioavailability
}

Kathia a. Sandoval

Florida International University, ksand002@fiu.edu

DOI: $10.25148 /$ etd.FIDC000258

Follow this and additional works at: https://digitalcommons.fiu.edu/etd

Part of the Chemistry Commons

\section{Recommended Citation}

Sandoval, Kathia a., "Physical and Chemical Characterization of Crude Oil-Water Mixtures: Understanding the Effects of Interfacial Process to Chemical Bioavailability" (2016). FIU Electronic Theses and Dissertations. 2464.

https://digitalcommons.fiu.edu/etd/2464 


\section{FLORIDA INTERNATIONAL UNIVERSITY}

Miami, Florida

\section{PHYSICAL AND CHEMICAL CHARACTERIZATION OF CRUDE OIL-WATER MIXTURES: UNDERSTANDING THE EFFECTS OF INTERFACIAL PROCESS TO CHEMICAL BIOAVAILABILITY}

A thesis submitted in partial fulfillment of the requirements for the degree of MASTER OF SCIENCE in CHEMISTRY

by

Kathia Sandoval

2016 
To: Dean Michael R. Heithaus

College of Arts, Sciences and Education

This thesis, written by Kathia Sandoval and entitled Physical and Chemical Characterization of Crude Oil-water Mixtures: Understanding the Effects of Interfacial Process to Chemical Bioavailability, having been approved in respect to style and intellectual content, is referred to you for judgment.

We have read this dissertation and recommend that it be approved.

$\begin{array}{r}\hline \text { Rudolf Jaffe } \\ \hline \text { John Landrum } \\ \hline \text { Piero R. Gardinali, Major Professor }\end{array}$

Date of Defense: March 30, 2016

The thesis of Kathia Sandoval is approved.

Dean Michael R. Heithaus College of Arts, Sciences and Education

Andrés G. Gil Vice President for Research and Economic Development And Dean of the University Graduate School

Florida International University, 2016 
(C) Copyright 2016 by Kathia Sandoval

All rights reserved. 


\section{DEDICATION}

I dedicate this thesis to my mother Ana Tello for her unconditional love and support throughout this journey. Also to my love ones: Sayuri Sandoval, Benjamin Sandoval, Sebastian Sandoval, Samuel Sandoval, Sarah Faith Sandoval and Laura Rivero. For their love, understanding, support, and good humor which encouraged me to complete this work. 


\section{ACKNOWLEDGMENTS}

First and most, I would like to thank my major professor, Dr. Piero R. Gardinali for giving me the opportunity to work with him and most important for believing in me. His guidance, patience and support through all these years have allowed me to grow as a scientist and I'm extremely grateful for that. Also, I would like to thank my committee members Dr. Jaffé and Dr. Landrum for their time and helpful advice of my research work.

I am very thankful to have been a part of EARL as I learned so much, met so many great people and developed long lasting friendships. Special thanks to Ingrid for her caring nature and encouraging words. To Adolfo for been the best lab manager one can ask for. To Anna Katarina and Karina for been my good friends and partners in crime. Also, to all the other wonderful people that have helped me one way or another: Cesar, Jenny, Margarita, Nubia, Gabriela, Carlos, Alina, Jian, Sudha, Venkat, Alan, Natalia, Chengtao and Raphael. I would also like to acknowledge SERC, the Department of Chemistry \& Biochemistry and Florida International University for their support. 


\begin{abstract}
OF THE THESIS
PHYSICAL AND CHEMICAL CHARACTERIZATION OF CRUDE OIL-WATER MIXTURES: UNDERSTANDING THE EFFECTS OF INTERFACIAL PROCESS TO CHEMICAL BIOAVAILABILITY

by
\end{abstract}

Kathia Sandoval

Florida International University, 2016

Miami, Florida

Professor Piero R. Gardinali, Major Professor

This work detailed the physical and chemical characterization of oil water mixtures prepared using fresh and weathered Macondo related oils under different conditions of mixing energy/time and in the presence/absence of chemical dispersants. The results indicated that WAFs produced consistent, droplet free solutions for both source and weathered oils with concentration ranges that represented the soluble components of the oil used. Chemically enhanced WAFs prepared with the source oil generated a large amount of micron-size droplets; however the viscosity of the weathered oils were a limiting factor for the preparation of CEWAFs with weathered oils. Droplet size distributions were influenced by the amount of energy in the system and the oil weathering stage, when high energy WAFs were made the increase in weathering of the oil resulted in the formation of smaller droplets that were more stable over time. 


\section{TABLE OF CONTENTS}

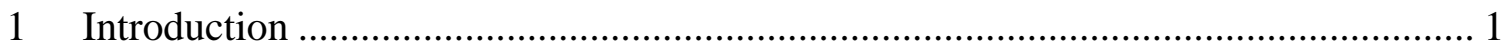

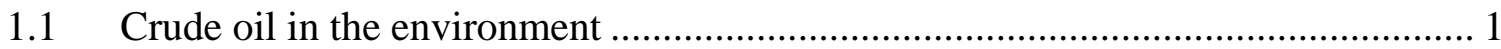

1.1.1 Deepwater Horizon oil spill in the Gulf of Mexico ............................................ 1

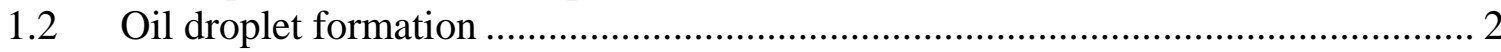

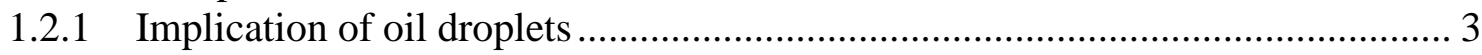

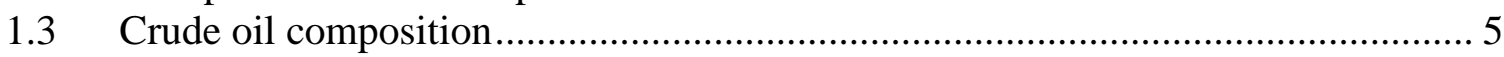

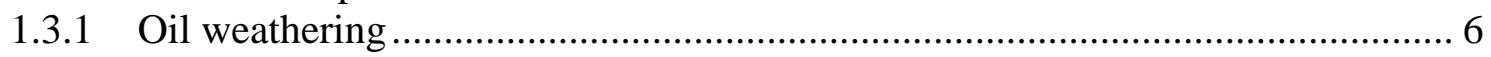

$1.4 \quad$ Exposure media used for toxicity test............................................................. 6

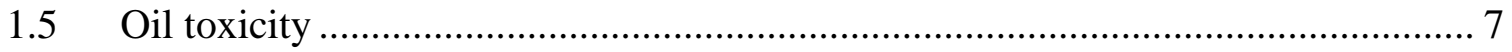

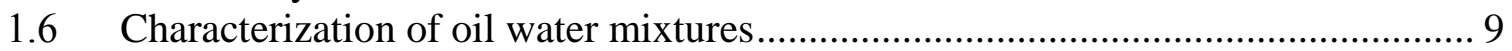

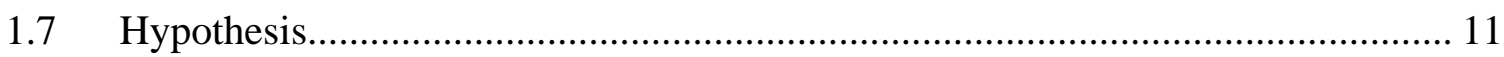

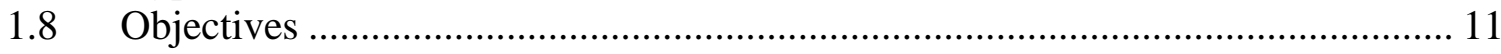

2 Characterization and environmental relevance of oil water preparations of fresh and weathered MC252 Macondo oils used in toxicology testing ..................................... 12

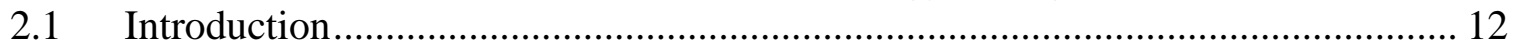

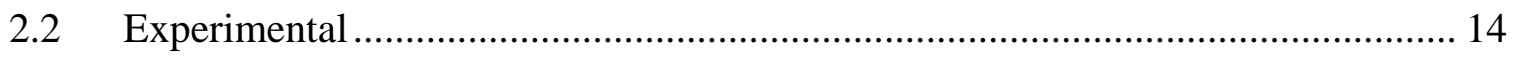

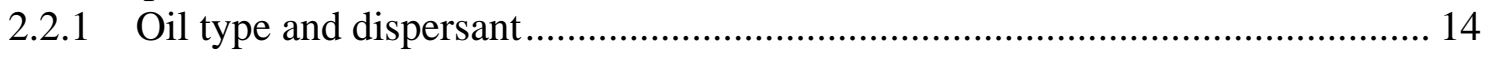

2.2.2 Preparation of water accommodated fractions (WAFs) and chemically enhanced water accommodated fractions (CEWAFs) ................................................. 15

2.2.3 Preparation of high energy water accommodated fractions (HEWAFs) and super high energy water accommodated fractions (SHEWAFs).................................... 16

2.2.4 Preparation of oil water dispersions (OWDs) ……........................................ 17

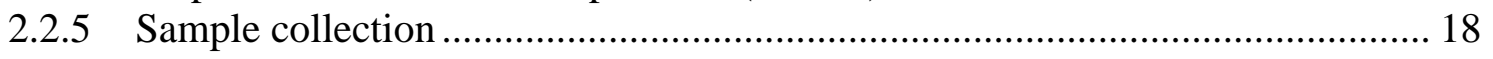

2.2.6 Droplet characterization using Epi-Fluorescence microscopy ............................ 18

2.2.7 Droplet size distribution (DSD) analysis using a particle size analyzer (Coulter Counter)...

2.2.8 Characterization method for dissolved and entrained oil components in the oil water treatments by Fluorescence excitation emission matrix spectroscopy (EEMs) ... 21 2.2.9 Chemical characterization: Total petroleum hydrocarbons by Fluorescence

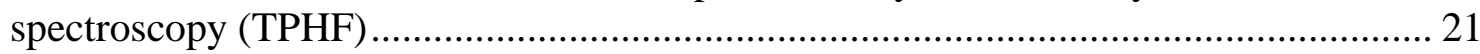

2.2.10 Chemical characterization: Extraction method ............................................. 22

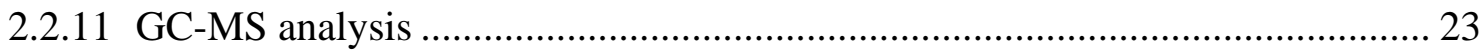

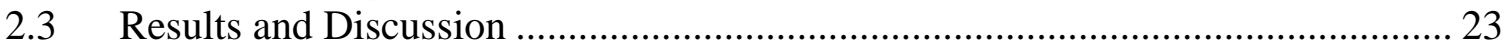

2.3.1 Droplet characterization ................................................................................. 23

2.3.2 Physical characterization of dissolved versus droplet phase by Fluorescence spectroscopy ............................................................................................................... 27

2.3.3 Effects of weathering on physical characteristics of the oil-water mixtures...... 30

2.3.4 Effects of oil loading on droplet size for high energy preparations .................... 33

2.3.5 Dilution series of oil water dispersions (OWDs) prepared with MASS oil ....... 37

2.3.6 Stability of different oil loading preparations with time .................................... 39 
2.3.7 Chemical characterization of exposure media preparations................................ 41

2.3.8 Chemical stability of oil preparations with time ……….................................. 43

2.3.9 Effects of filtration for the preparation of water soluble fractions (WSFs) ....... 45

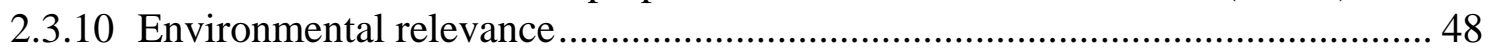

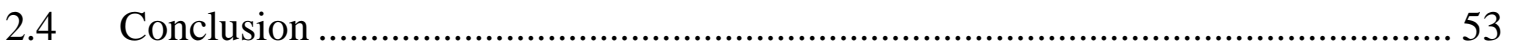

3 A novel delivery system for studying the dissolution of individual and combined polycyclic aromatic hydrocarbons (PAHs) from micron-size droplets ............................ 55

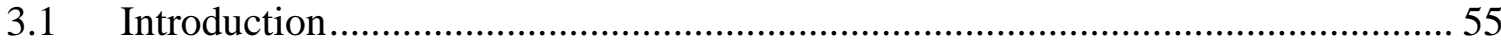

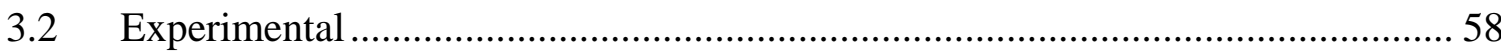

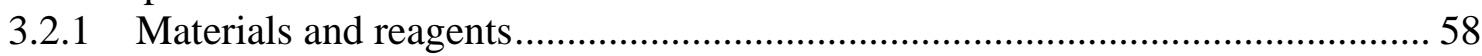

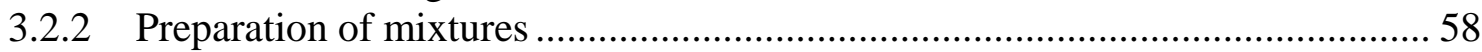

3.2.3 Analysis of mixtures by GC-FID and GC-MS .......................................... 59

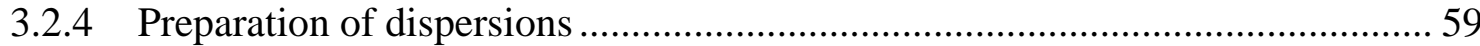

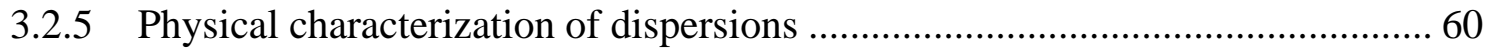

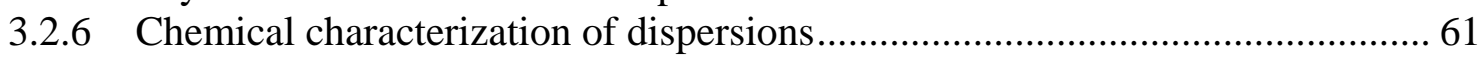

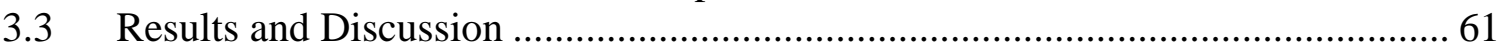

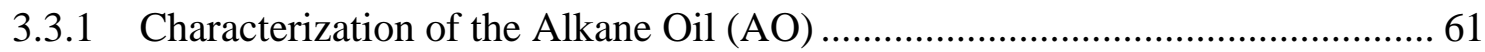

3.3.2 Droplet characterization of the oil water dispersions (OWDs) prepared with

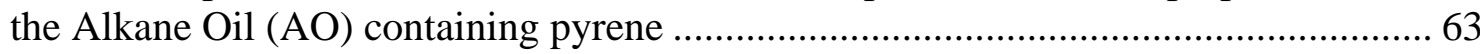

3.3.3 Fluorescence characterization of water soluble fractions (WSFs) and oil water

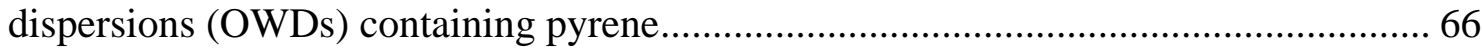

3.3.4 Chemical characterization of oil water dispersions (OWDs) prepared with the Alkane Oil (AO) containing Pyrene

3.3.5 Dissolution and equilibration of individual polycyclic aromatic hydrocarbons

(PAHs) from the $\mathrm{AO}$ droplets into the water phase ……………………………........... 70

3.3.6 Characterization of PAH mixture-AO and a well-studied crude oil .................. 75

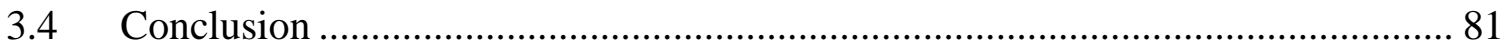

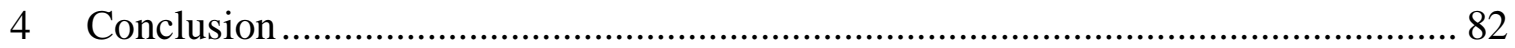

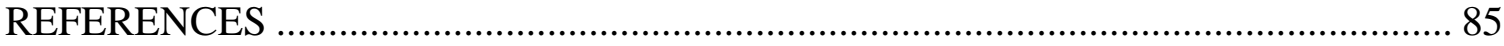

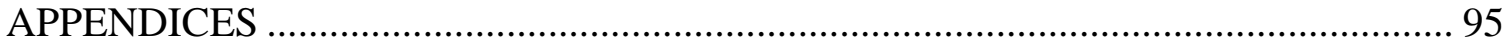




\section{LIST OF FIGURES}

\section{FIGURE}

Figure 1: Output model of the trajectory of different droplet sizes from a deepwater oil release reported in (Yapa et al., 2012)................................................................... 4

Figure 2: Physical characterization of different treatments (water accomodated fractions (WAF), chemically enhanced water accomodated fractions (CEWAF), high energy water accomodated fractions (HEWAF) and super high energy water accomodated fractions (SHEWAF)) prepared with MASS oil, Epi-fluorescence microscope images (top) and Coulter Counter histograms (bottom) measured at initial preparation time after settling

Figure 3: Physical characterization of different treatments(water accomodated fractions (WAF), chemically enhanced water accomodated fractions (CEWAF), high energy water accomodated fractions (HEWAF) and super high energy water accomodated fractions (SHEWAF)) prepared with CTC oil, Epi-fluorescence microscope images (top) and Coulter Counter histograms (bottom) measured at initial preparation time after settling....

Figure 4: Fluorescence excitation emission matrix (EEM) spectra showed as contour plots for the water accommodated fraction (WAF) (top) and the chemically enhanced water accommodated fraction (CEWAF) (bottom) preparations using the source MASS oil. The red diagonal lines across the EEMs correspond to the Rayleigh and Raman scattering peaks.

Figure 5: Influence of oil weathering in terms of specific gravity (MASS $=0.84$, $\mathrm{CTC}=0.98$, and Juniper $=1.00)$ on the volume median diameter $(\mu \mathrm{m})$ of droplets measured by Coulter Counter for the high energy preparations.

Figure 6: Volume median diameter $(\mu \mathrm{m})$ measured by Coulter Counter for different nominal oil loadings (top) and dilution series of a 1000 ppm stock solution (bottom) for SHEWAFs prepared with CTC oil.

Figure 7: Relationship between nominal and measured concentrations (top) where TPHF measurements are shown in red and Coulter Counter measurements are shown in blue for SHEWAFs prepared with CTC oil and the corresponding trends with droplet sizes (bottom) showing a linear dependency between decreasing measured loading concentrations and particle size distributions.

Figure 8: Droplet size distributions (based on volume) obtained from Coulter Counter analysis for the dilution series of OWDs $(2,5,15,20,60 \mathrm{mg} / \mathrm{L}$, top). Comparison of oil concentration measurements by Coulter Counter and total petroleum hydrocarbons by fluorescence of the dilution series (bottom). 
Figure 9: Stability of the SHEWAF preparations with CTC oil at different oil loadings measured as TPHF over a 96 hour period.

Figure 10: PAH profiles and concentrations for MASS (left) CTC (middle) and JUNIPER (right) oils preparations at initial collection time for all treatments (water accomodated fractions (WAF), chemically enhanced water accomodated fractions (CEWAF), high energy water accomodated fractions (HEWAF) and super high energy water accomodated fractions (SHEWAF)). Abbreviations for PAHs are described in appendix 1

Figure 11: Stability of WAF preparations (top) and HEWAF preparations (bottom) measured as total polycyclic aromatic hydrocarbons (TPAH) concentrations over 96 hours for all oils, MASS $=$ triangle, $\mathrm{CTC}=$ circle and Juniper= square. The inset shows an expanded view for the Juniper oil due to the overall lower concentrations present in the high energy preparation.

Figure 12: Comparison of WSF to WAF for all treatments and oils. Solid black line indicates $100 \%$ of WAF and dash black lines are $\pm 30 \%$ boundaries.

Figure 13: Total PAH concentrations in field samples collected from the Gulf of Mexico over time (May 2010 to December 2010) and TPAH concentrations for all treatments and oils in log scale.

Figure 14: Sum of the concentration of 2-3 ring PAHs (left) and sum of the concentration of 4-6 ring PAHs (right) for field collected samples from the Gulf of Mexico and all the treatments prepared with all the oils in linear scale.

Figure 15: Chromatogram of the Alkane Oil (AO) obtained by GC-FID showing the distribution of the alkanes and labeled internal standards.

Figure 16: Microscope images of Alkane Oil (AO) droplets for three concentrations of pyrene in the AO at a) $163 \mathrm{ppm} \mathrm{b} 827 \mathrm{ppm}$ and c) $1855 \mathrm{ppm}$ in bright field (top) and UV fluorescence illumination (bottom). The inlet images are zoomed in of the droplets corresponding to the droplets that are circled in the bright field images....

Figure 17: Contour plots for the filtered WSF (top) and unfiltered OWD (bottom) for dispersions prepared with AO-PYR $(827 \mathrm{ppm})$. The diagonal red lines are due to first and second order Rayleigh scattering produced by the instrument and not related to the data.

Figure 18: Relationship between the fluorescence intensity and concentration of pyrene in AO for the WSF (A) and OWD (B). Solid line is linear regression and dashed line is $\pm 95 \%$ confidence limits. 
Figure 19: Comparison between the measured and predicted concentration of total pyrene (sum in both dissolved and droplet phase) for the dispersions prepared with different amounts of pyrene in the $\mathrm{AO}$.

Figure 20: Dissolved aqueous phase concentrations versus increased mole fraction in the Alkane Oil (AO) for fluorene (top), pyrene (middle) and chrysene (bottom)....72

Figure 21: Comparison of $\log \mathrm{S}$ versus $\log$ Kow at constant mole fraction of the individual PAHs in the AO.

Figure 22: Distribution of the concentration of PAHs in both particulate and dissolved phase for the Macondo source oil and the alkane oil.

Figure 23: Predicted versus measured dissolved phase concentrations of PAHs present in the water-soluble fractions (WSFs) prepared with the Alkane Oil. The 2-ring aromatics are shown as $(\mathbf{\Lambda})$, 3-ring PAHs $(\bullet)$ and 4-ring PAHs (•). The solid and dashed lines indicate $1: 1$ and 2:1 or 1:2 lines, respectively.

Figure 24: Predicted versus measured dissolved phase concentrations of PAHs measured from the water soluble fractions (WSFs) prepared with the Macondo source oil. The solid line indicate the 1:1 line calculated using the $\mathrm{MW}=187 \mathrm{~g} / \mathrm{mol}$ and the dashed line indicate the 1:1 line calculated using the best-fit $\mathrm{MW}=272$ $\mathrm{g} / \mathrm{mol}$. 


\section{LIST OF ABBREVIATIONS AND ACRONYMS}

ABBREVIATION

$\mathrm{AO}$

CEWAF

CROSERF

DCM

DMSO

DSD

DWH

EEM

$\mathrm{Ex} / \mathrm{Em}$

GC-MS

GC-FID

GOM

HEWAF

Kow

LC50

LISST

LLE

MAH

MC

MDLs

$\mathrm{MW}_{\mathrm{av}}$
FULL NAME

Alkane oil

Chemically enhanced water accommodated fraction Chemical response to Oil Spills: Ecological Effects Research Forum Dichloromethane Dimethyl Sulfoxide Droplet size distribution Deepwater Horizon Excitation-Emission matrix Excitation/ Emission Gas chromatography-Mass spectrometry chromatography Gas chromatography-Flame ionization detector

Gulf of Mexico

High energy water accommodated fraction Octanol-water partitioning coefficient

Lethal median concentration Laser in-situ scattering and transmissometry Liquid-liquid extraction Mono aromatic hydrocarbons Mississippi Canyon Method detection limits Average molecular weight 
PAHs Polycyclic Aromatic Hydrocarbons

PFI Pressurized flow injection generator ppm Parts per million

$\mathrm{ppb}$ Parts per billion

QqQ Triple quadrupole

RPD Relative percent difference

SIM

Selective ion monitoring

SHEWAF

Super high energy water accommodated fraction

SPE-LC-APPI-MS/MS

Solid phase extraction-Liquid Chromatography-Atmospheric pressure photoionization-Mass spectrometry/mass spectrometry

$\mathrm{TPH}$

Total petroleum hydrocarbons

TPHF

Total petroleum hydrocarbon by fluorescence

TU

Toxic units

UV

Ultra violet

WAFs

Water accommodated fractions

WSFs

Water soluble fractions

VMD

Volume median diameter 


\section{Introduction}

\subsection{Crude oil in the environment}

Oil is a natural product that is undoubtedly one of the most complex environmental mixtures known to man (Marshall and Rodgers, 2004). Oil is generated through diagenesis of its source organic matter resulting on a final product composed of a myriad of relatively non-polar components with great diversity of structures, functionalities and chemical properties such as volatility, solubility and polarity (Tissot and Welte, 1984). Over a million metric tons of crude oil are released into the marine environment annually from accidental oil spills, industrial/municipal sources, marine transport and natural oil seeps (Radovic et al., 2014). Oil released into the sea will produce heterogeneous and diversified mixtures of dissolved hydrocarbon constituents and undissolved oil residues such as oil droplets with different physical/chemical properties which can be affected by a wide range of environmental factors. Thus, oil is not only a complex system but also a dynamic one that is not at equilibrium with the surrounding environment.

\subsubsection{Deepwater Horizon oil spill in the Gulf of Mexico}

The Deepwater Horizon (DWH) incident in the northern Gulf of Mexico (GOM) deemed as the second largest global oil spill in history, resulted in the release of large quantities of Southern Louisiana light sweet crude oil as an oil-gas mixture from the Macondo MC-252 (Mississippi Canyon-Lease block 252) well at a depth of $1500 \mathrm{~m}$ (Reddy et al., 2012; Ryerson et al., 2012). Several sources have reported an estimate release of approximately 4.9 million barrels of oil (Spier et al., 2013; Thibodeaux et al., 
2011). Mixing of the oil and water resulted in a heterogeneous mixture consisting of dissolved, dispersed and solid oil components. During response efforts, the chemical dispersants, Corexit ${ }^{\circledR}$ EC9500a and to a lesser extent Corexit ${ }^{\circledR}$ EC9700a, were applied on the surface and in the subsurface at the wellhead, in order to promote the formation of small oil droplets that could be subject to enhanced dissolution and biodegradation in the water column in order to prevent large amounts of oil from reaching the shorelines ( $\mathrm{Li}$ et al., 2011).

\subsection{Oil droplet formation}

During a surface oil spill event, droplet formation occurs as a result of the mechanical or chemical break-up of an oil slick into small droplets dispersed into the water column. Natural oil dispersion is mainly influenced by mechanical processes which are governed by physical factors such as wind, currents and wave action (Zhong and You, 2011). Chemically enhanced dispersions make use of chemical dispersants to lower the oil-water interfacial tension by orienting the interaction of hydrophilic groups with the water phase and hydrophobic groups with the oil so that formation of small oil droplets require less energy input (NRC, 2005). The characteristics of the oil droplets such as size distribution, density, and composition depend on key parameters such as the physical and chemical properties of the oil, use of chemical dispersants, the turbulent energy at the release point, and the water temperature and pressure at the release location (Delvigne, 1987). For instance, Trudel et al. (2010) showed that the viscosity of oil influences the chemical dispersant effectiveness and droplet formation as less viscous oil produced very small droplets (range of 8-37 $\mu \mathrm{m}$ ) while more viscous oils produced larger droplets (60$73 \mu \mathrm{m})$. 
In a deep-water scenario such as the one that occurred during the DWH oil spill, the presence of gas in underwater oil releases will break up into a mixture of gas bubbles and oil droplets of various sizes. Initially, a mixture of plume fluid driven by the plume dynamics containing all phases (oil, gas, water and hydrates) will cluster together and move in unity (Yapa et al., 2012). However, after settling of the plume dynamics the movement of each phase will be driven by their own buoyant velocity. Since gases are lighter than oil and water, they will travel faster to the surface and dissociate or dissolve in the water column much quicker while oil droplets which are denser will rise towards the surface at a rate that is dependent on their size, density and the conditions of the seawater. Large droplets are expected to reach the surface fairly quickly while smaller oil droplets may stay in the water column for extended periods of time before reaching the surface (Atlas and Hazen, 2011; Baelum et al., 2012; Hazen et al., 2010).

\subsubsection{Implication of oil droplets}

The formation of oil droplets is particularly important because oil droplet sizes will influence the transport of oil, the oil dissolution and the oil mass. For instance, Yapa et al. (2012) described the trajectory of droplets of different sizes using a model with parameters similar to the deepwater oil release which occurred in the GOM as shown in Figure 1. The larger droplets with diameters of 5 to $6 \mathrm{~mm}$ go up the surface fairly quickly within a couple of hours while sub-micron size droplets take much longer time to go up the surface and droplets which are smaller than $100 \mu \mathrm{m}$ are most likely to become buoyant and be subject to lateral transport across the water column by currents. 


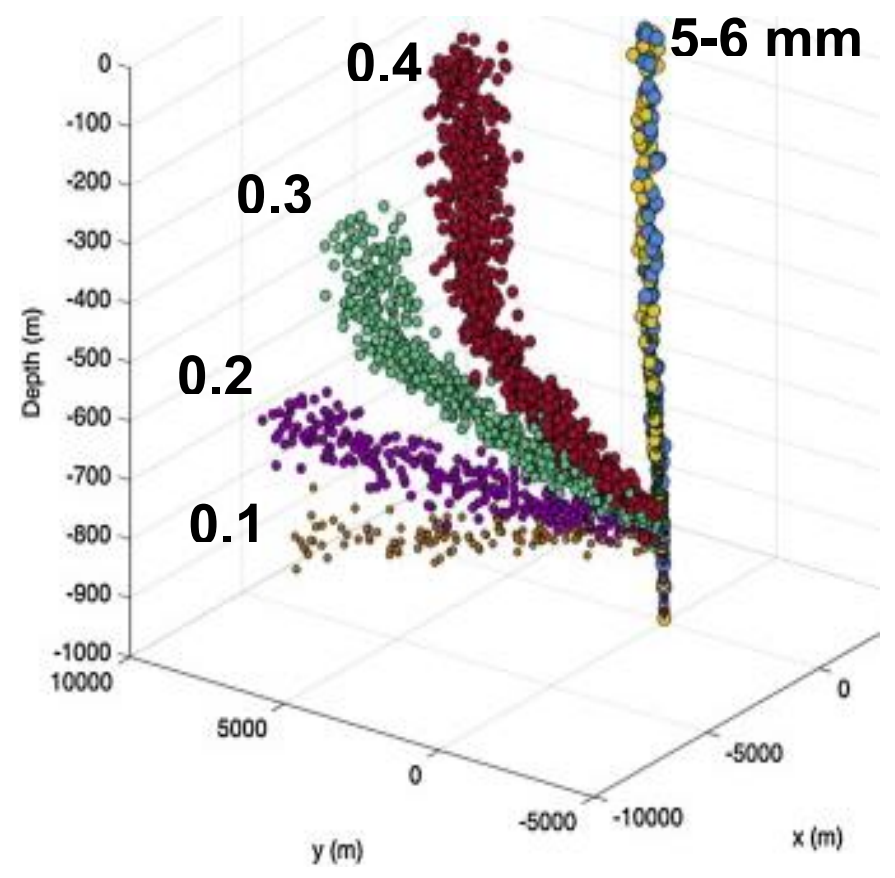

Figure 1: Output model of the trajectory of different droplet sizes from a deepwater oil release reported in (Yapa et al., 2012).

Small oil droplets have an increased surface to volume ratio which results in the rapid dissolution of highly soluble oil components into the water column. For example, measurements of the water column during the DWH oil spill for benzene, a highly water soluble oil compound, showed a systematic elevated concentration $(0.4-21.7 \mu \mathrm{g} / \mathrm{L})$ at a depth of 1,100 $\mathrm{m}$ and no detection at shallower depths than 1,000 $\mathrm{m}$ (Reddy et al., 2012). In addition, oil droplet sizes will influence the mass of the oil or bulk concentration present in the water column as larger droplets indicate a larger volume of oil present in comparison to smaller droplets. Chemical data from the DWH oil spill indicated that the large depletion of heavier n-alkanes (mostly present in the particulate phase) relative to toluene (highly soluble) demonstrated that little mass was transported into the deep plumes in the form of suspended oil droplets (Ryerson et al., 2012). 


\subsection{Crude oil composition}

Crude oil is a complex mixture containing thousands of compounds from several different chemical classes with a large variety in physicochemical properties (Boehm et al., 1997). The chemical composition of crude oils is generally divided into four classes of chemical groups on the basis of their characteristics. These include saturated hydrocarbons, aromatics, resins and asphaltenes. The saturated hydrocarbons are compounds such as straight-chain/branched alkanes (octane, pristine), paraffins (methane, propane), and cycloalkanes (naphtenes). These are the simplest hydrocarbon compounds and lightest fraction of the crude oil. Aromatics hydrocarbons can be either mono, di or polycyclic such as benzene, naphthalene and fluorene. Resins are highly polar compounds that contain heteroatoms (oxygen, sulfur or nitrogen) such as quinolones or naphthenic acids and they are soluble in light alkanes but insoluble in aromatic solvents, while asphaltenes are similar to resins but are higher in molecular weight, their structures are highly complex and they precipitate in light alkanes and are soluble in aromatic solvents (Speight, 2004).

Crude oils are further classified as either light, medium and/or heavy depending on their density in relation to their specific gravity value (NRC, 2005). Their density is apportioned to the concentrations of the different components in the oil. Oils which are light will mostly contain saturated hydrocarbons and lower molecular weight aromatics, while medium and heavy oils will contain a large portion of aromatics, resins and asphaltenes. In addition, crude oils that contain a high sulfur content (more than $1 \%$ sulfur by weight) are considered 'sour', while those that contain lower than $1 \%$ sulfur content are considered 'sweet' (Lyons and Plisga, 2011). 


\subsubsection{Oil weathering}

From the moment crude oil is removed from its original reservoir or as it is released into the environment its physical and chemical composition changes dramatically as the result of complex interactions with environmental compartments and their interfaces (Neff, 1988). The change in composition is termed "oil weathering" and some of the most important weathering processes consist of evaporation, dissolution, emulsification, and photo-oxidation (Yim et al., 2011). Evaporation involves the loss of lower molecular weight volatile components of the oil mixture into the air. Dissolution partitions the soluble and sparingly soluble components of the oil into the water column until equilibrium between the aqueous and oil phase or saturation is reached. Photochemical reactions occur as a result of photooxidation of the chemicals in the oil with sunlight exposure to generate oxygenated reaction products (Farrington, 2014). These processes often lead to significant changes in the physicochemical properties of the oil, especially in the viscosity of the oil and are important to consider because of their implication in the fate and transport of the spilled oil (Zhong and You, 2011).

\subsection{Exposure media used for toxicity test}

Toxicology testing is one of the primary tools used to assess the impacts of an oil spill on the marine ecosystem. Bench-scale acute toxicity tests are often utilized to study the effects of dispersed oil and consist of exposing an organism to dispersed oil preparations that are prepared under controlled laboratory conditions (NRC, 2005). There are many parameters that could influence the toxicity test and need to be carefully examined. Some of these parameters consist of the following: (1) The state of the oil such as whether is fresh or weathered, and the degree of weathering; (2) The preparation 
method of the exposure media used to expose organisms; (3) The exposure settings such the choice of test chambers, static or continuous exposure, test duration, temperature and salinity and (4) the choice of response parameters (NRC, 2005). Additionally, it is important for the concentrations of the oil components to which the test organisms are expose to be environmentally realistic, thus the methodology used for exposure media preparation is critical as changes to the mixing energy or even the oil loading can drastically affect the concentrations of the oil components.

The Chemical Response to Oil Spills Environmental Research Forum (CROSERF) developed and standardized methods for the preparation of exposure media used for toxicity test that would allow for better comparability of toxicology data. These methods reported by Singer et al. (2000) consist of the water accommodated fractions (WAFs) produced at low mixing energies and the chemically enhanced water accommodated fractions (CEWAFs) created by using chemical dispersants. The first usually represents the water soluble components of an oil-water mixture while the latter is used as a representation of a combined mixture of both WAF and oil droplets. Recently, new preparation of standardized exposure media containing entrained oil in the absence of chemical dispersants have been reported in the literature and their description varies from oil-water dispersions (OWDs) prepared with a pressurized fluid injection generator (Nordtug et al., 2011a) to high-energy water accommodated fractions (HEWAFs) created by manual or mechanical mixing (Carls et al., 2008; Echols et al., 2015).

\subsection{Oil toxicity}

The toxicity of oil has long been attributed to its soluble components because they are more bioavailable to the organisms (Anderson, 1985; Anderson et al., 1987) and their 
toxic contribution depend on the composition of the oil as it will influence the concentration of dissolved phase hydrocarbons (Redman et al., 2012a). Predictive models accounting for the wide range of toxicity of petroleum hydrocarbons based on the narcosis target lipid model (NTLM) have been developed and validated (Di Toro and McGrath, 2000; French-McCay, 2002; McGrath and Di Toro, 2009; Redman et al., 2012b). The NTLM predicts the toxicity of a group of compounds that exert the same toxic mode of action (narcosis) and uses the inverse relationship observed between the lethal concentration to $50 \%$ of the organisms (LC50) and the octanol-water portioning coefficient (Kow) (McGrath et al., 2005). In general, the low molecular weight mono aromatic hydrocarbons and the 2-3 ring parent and alkyl-PAHs dominate the toxicity of oil and their weighed contribution can be predicted using the toxic unit (TU) approach (Di Toro and McGrath, 2000; Landrum et al., 2003). Under this concept, the concentrations of soluble oil components are estimated using Raoult's Law which states that the aqueous solubility concentration of a compound in the oil is equal to the aqueous solubility of the pure liquid multiplied by its mole fraction in the oil (Di Toro et al., 2007). Thus, the maximum toxicity a chemical can exert depends on its maximum aqueous concentration. Because of this, most of the toxicology tests focus mainly on the dissolved oil components. However, it is important to differentiate the uptake of the oil between the dissolved and oil droplet phase as toxic effects could be induced from a combination of several modes of exposure.

Oil droplets can induce toxicity to marine organisms by both physical and chemical processes (Ramachandran et al., 2006; Ramachandran et al., 2004a; Viaene et al., 2014). They could act as physical stressors through oil coating or be ingested as food 
which could cause blockage in the gut. They could provide a delivery mechanism for toxic compounds into the dissolved phase (monoaromatics and PAHs) and also interact with biological surfaces and thus enhance bioavailability. For instances, many studies have found an increase in toxicity for exposure media preparations consisting of CEWAFs or OWDs which contained droplets in comparison to WAFs because of higher concentration of bioavailable (dissolved and colloidal) PAHs in the dispersions (Couillard et al., 2005; González-Doncel et al., 2008; Ramachandran et al., 2004a). Contact with the oil droplets can lead to partition directly into the organism's lipid-rich tissues such as fish eggs and gills as those are natural absorbents for lipophilic PAHs and can be bioaccumulated (Ramachandran et al., 2004b). Other studies have suggested that the main effect from oil droplets is an additional input of dissolved hydrocarbons into the exposure media (Redman, 2015).

\subsection{Characterization of oil water mixtures}

Very few studies have shown a comprehensive physical characterization of the particulate oil in the test media and the methods used for this purpose often lack of standardization (microscopy) (Incardona et al., 2013), do not have enough selectivity towards oil droplets (Coulter Counter particle analyzer) (Hansen et al., 2009; Nordtug et al., 2011a), are limited by the technology used (Laser In-Situ Scattering and Transmissometry, LISST) (Conmy et al., 2014; Li et al., 2011; Li et al., 2009; Trudel et al., 2010) or conducted after the fact by complex solubility calculations once the full chemical characterization is completed (Redman et al., 2012a). All these limitations prevent timely feedback on the evolution of the mixtures before, during and after the test 
which could be used to manage the longer periods of time required for chronic toxicity testing ( $>7$ days).

Chemical characterization of the exposure media used to evaluate toxicity endpoints consist of measuring the total petroleum hydrocarbon content (TPH) by summing the concentrations of $\mathrm{C}_{10}-\mathrm{C}_{36}$ hydrocarbons analyzed by gas chromatography with flame ionization detection (GC-FID) (Singer et al., 2000). In other cases, parent and alkylated PAHs are analyzed by gas chromatography-mass spectrometry (GC-MS) and often reported as the concentration of total polycyclic hydrocarbons (TPAH) from the analysis of typically 40-50 target compounds (Barron and Ka'aihue, 2003). Expressing toxicity in term of TPH or TPAH without providing detailed information on the composition of the mixture or the concentrations of individual components has not only affected toxicity interpretations but it has also made cross-comparison in toxicology studies extremely challenging (Bejarano et al., 2014).

Furthermore, oil toxicity test exposures that report measured concentrations of individual components as a combination of both dissolved and droplet form may lead to overestimation for the potential of the solution to cause chemical toxicity (Redman, 2015). Thus, it is important to take into account the presence of entrained oil droplets in an exposure scenario as it may introduce confounding physical and chemical factors that need to be separated from those related exclusively to the dissolved compounds. Therefore, in order to properly assess toxicity it is crucial to provide a comprehensive chemical and physical characterization of the aqueous exposures used in laboratory studies such as the measurement of individual PAHs, presence of oil droplets, and the distribution of the individual components in the different phases of the mixture. 


\subsection{Hypothesis}

Droplet formation will be controlled by the mixing energy applied to the system and droplet size distribution will be the most important factor in dispersion stability.

The physical and chemical composition of oil water mixtures will differ among oil type and weathering degree.

The solubility of the dissolved components in the water soluble fractions (WSFs) of the oil water mixtures will be governed by Raoult's law.

\subsection{Objectives}

The overall objectives of this work are to determine how different parameters (loading, oil type, energy of mixing and/or filtering conditions) used for the preparation of oil water mixtures affect the composition of exposure media used for toxicological testing and to describe the application of a new dosing system for the delivery of toxicants of concern.

In order to accomplish this task, specific aims include:

- Develop a set of combined tools to properly characterize the composition of different oil water mixtures in real time.

- Assess the formation and removal of droplets and their stability with time in reference to toxicological testing conditions.

- Investigate the difference in chemical composition between the dispersions, oil droplets and WSFs.

- Develop a dosing system to deliver dissolved components to aqueous media at concentrations similar to the ones of a natural crude oil. 


\section{Characterization and environmental relevance of oil water preparations of fresh and weathered MC252 Macondo oils used in toxicology testing}

\subsection{Introduction}

Most of oil toxicity testing deals with exposing organisms to laboratory prepared

oil water mixtures using the standard protocols prepared by CROSERF and reported by Singer et al. (2000) consisting mainly of water accommodated fractions (WAFs) produced at low mixing energies and chemically enhanced water accommodated fractions (CEWAFs) created by using chemical dispersants. Recently, new methods for the preparation of high-energy water accommodated fractions (HEWAFs) which consist of the formation of oil containing exposure media in the absence of chemical dispersants for both source and weathered oils have been described (Brette et al., 2014; Brewton et al., 2013; Incardona et al., 2013; Jung et al., 2013; Mager et al., 2014). These methods, invariably involve turbulent mixing of oil in seawater followed by a settling period to remove resurfacing oil droplets from the exposure media. The HEWAF preparations using a commercial blender do provide the opportunity to create droplet-containing exposure media with weathered oils that are not easily dispersible but its implementation and application are highly dependent in its proper standardization and characterization since most reports in the literature do not seem to reflect a single protocol with respect to basic parameters such as mixing times or oil loadings as reflected by the oil to water ratios (Brette et al., 2014; Incardona et al., 2013; Mager et al., 2014). 
Because the opportunities to assess environmental impacts in the field during an incident are very limited, the assessment of potential effects during an oil spill are predominantly subject to our ability to generate exposure conditions that mimic those existing at the test site in a controlled laboratory setting (Bejarano et al., 2014). The basis of assessing an effect relies on the ability to fully understand the identity of the material components (what), the dose (how much), the duration (how long) and the route, pathway or mechanism by which exposure occurs. These conditions should also be relevant to a particular organism or biological resource, representative of a time and location during the event and predictive enough so it could be extrapolated to larger areas where information data gaps exist.

Even though extensive reviews of the toxicological assessment of oil have been conducted over the last two decades (Aurand and Coelho, 2005; Barron and Ka'aihue, 2003; Singer et al., 2000) discrepancies in the use of the methodologies and its interpretations still exists today (Bejarano et al., 2014; Coelho et al., 2013) and that is mostly the result of the lack of comprehensive characterization of the exposure media. For example, droplet formation, size distributions and densities are rarely considered relevant factors during toxicological testing of crude oils. However, droplet formation is an important process that occurs during oil spills. The limited reports on the potential effects of droplets, present in mechanically or chemically driven oil water dispersions range from apportioning all the observed effects to water soluble components (Carls et al., 2008; Viaene et al., 2014) to minor effects shown at very high droplet concentrations (Nordtug et al., 2015) to size-dependent droplet derived effects (Bobra et al., 1989) and increased PAH bioavailability from CEWAFs (Ramachandran et al., 2004a). Clearly, it is 
important to account for oil droplets when assessing oil toxicity. Empirical data on the droplet size distribution (DSD) in the GOM water column during the DWH event were very limited and only estimates of approximately $20 \mu \mathrm{m}$ (less than $70 \mu \mathrm{m}$ ) have been reported (Li et al., 2011). With this limited information, addressing a target droplet size when assessing the effects of entrained oil during toxicity testing is an estimate at best.

In the present study we describe, compare and contrast traditional (WAF and CEWAF) and recently described (HEWAF, SHEWAF, and OWD) batch preparation methods of exposure media produced with Macondo related oils (source and naturally weathered) with respect to their chemical composition, the physical characterization of oil droplets, their stability over timescales relevant to common short-term toxicity testing and their environmental relevance with respect to water column data collected from the GOM during the DWH incident. This work is aimed to emphasize the advantages of a systematic and comprehensive physical and chemical characterization of exposure media to assess the potential shortcomings in their use for the assessment of biological effects.

\subsection{Experimental}

\subsubsection{Oil type and dispersant}

Three field-collected oils from the DWH response effort were used to prepare the treatments. These oils include (1) unweathered MC252 oil collected on August 15, 2010 from the Oil Barge Massachusetts (referred to herein as MASS); (2) slick A collected by skimmers from the GOM on July 19, 2010 from the cargo hold of barge number CTC02404 (referred to herein as CTC); and (3) slick B collected by skimmers on July 29, 2010 by the USCG Juniper (referred to herein as Juniper). Despite the different 
collection dates and likely as a result of the final location and environmental exposure of the slicks, the classification of the test oils in weathering order is MASS, a relatively fresh Macondo oil subject only to limited dissolution in the water column, followed by CTC with an overall depletion of PAHs (Bragg et al., 1994) with respect to the recalcitrant oil biomarker (30 $\alpha \beta$-hopane) of $65 \%$ and finally Juniper with an overall PAH depletion of $83 \%$ (BP, 2014). The composition of CTC oil is consistent with floating slicks while Juniper is more representative of the Macondo oil that reached the shoreline during the DWH incident. Dispersant-type Corexit ${ }^{\circledR}$ EC9500a (Nalco Inc., Sugarland, TX) was used to disperse the oils for the CEWAF treatments. Artificial filtered saltwater (pore size: $0.45 \mu \mathrm{m}$, salinity $=33 \mathrm{ppt}$ ) prepared with Instant Ocean ${ }^{\circledR}$ (Aquarium Systems, Mentor, $\mathrm{OH})$ was used to prepare all the treatments.

\subsubsection{Preparation of water accommodated fractions (WAFs) and chemically enhanced water accommodated fractions (CEWAFs)}

The WAFs and CEWAFs were prepared using the standardized CROSERF protocol described by Singer et al., (2000). The treatments were prepared in 2 liter glass aspirator bottles with $20 \%$ headspace by volume and covered with aluminum foil. Crude oil (MASS) was added onto the premeasured dilution water with a gas-tight syringe, while the more viscous oils (CTC and Juniper) were delivered by weight. The WAFs were prepared at nominal concentrations of $1 \mathrm{~g}$ oil/ $\mathrm{L}$ and allowed to mix for 24 hours using a 2 inch stirring bar and a Corning 10x10 digital stirring plate (Fisher Scientific, NJ) operated at low speed $(100 \mathrm{rpm})$ in order to saturate the solutions with all the watersoluble components and avoid the inclusion of particulate oil (no vortex mixing). 
The CEWAFs were made at the same oil loadings as the WAFs but with addition of dispersant to the surface of the oil-water interface using a $100 \mathrm{mg}$ glass syringe to achieve a 1:20 dispersant-to-oil ratio. The CEWAF preparations were allowed to mix for 18 hours at the same speeds $(100 \mathrm{rpm})$ and were allowed to settle for 6 hours in order for the larger oil droplets to resurface so the solution can be more homogeneous. After the end of the preparation period, the samples were collected from the bottom of the glass aspirator (to avoid collecting insoluble material at the water surface).

\subsubsection{Preparation of high energy water accommodated fractions (HEWAFs) and super high energy water accommodated fractions (SHEWAFs)}

The HEWAFs and SHEWAFs were prepared using a new protocol proposed by the National Oceanic and Atmospheric Administration (NOAA) and described for the first time in the literature by Incardona et al. (2013). Artificial filtered saltwater (volume of 3.5 liters) was placed inside a Waring CB15 commercial food blender (Torrington, CT) followed by delivery of the oil to achieve the desired loadings. The source oil was measured and delivered using a pre-cleaned gastight syringe onto the surface of the water inside the blender and covered with a custom made Teflon lid placed inside the blender that prevented splashing of the oil mixture into the plastic cap provided with the unit. Preparations requiring CTC and Juniper oils were made by measuring a known quantity of the corresponding weathered oil on an aluminum weighing boat and delivering the appropriate amount by weigh difference. HEWAFs preparations with the three oils were prepared at nominal concentrations of $1 \mathrm{~g}$ oil/L and blended for 30 seconds at the lowest speed setting $(\mathrm{LOW})(\sim 15000 \mathrm{rpm})$. 
Separate treatments of the weathered oils were made at varying nominal loadings with the same speed as HEWAFs or by increasing the mixing time to up to 2 minutes. In order to explore the effects of increased energy the preparations created with longer mixing times were defined as SHEWAFs. After preparation, the high-energy dispersions were immediately transferred into two (2L) separatory funnels and allowed to settle for 1 hour before collection.

\subsubsection{Preparation of oil water dispersions (OWDs)}

Mechanically created OWDs followed the pressurized fluid injection method described by Nordtug et al. (2011a). The generator consisted of a series of chambers with connecting nozzles which is capable of producing dispersions of consistent DSDs using turbulent flow mixing. Filtered saltwater was fed to the droplet generator at a designated constant flow using an FMI valveless metering pump Model QV fitted with an Stroke Rate Control Unit Model V300 (Fluid metering Inc., Syosset NY, USA). Crude oil (MASS) was added through an inlet capillary into the generator through a syringe needle using a Nexus 6000 high pressure syringe pump (Chemyx, Stafford TX, USA) fitted with a $10 \mathrm{ml}$ gas-tight syringe. The generator was operated at a flow rate of $142 \mathrm{ml} / \mathrm{min}$ for saltwater in order to produce approximately $10 \mu \mathrm{m}$ median droplet sizes on volume dispersions. The oil loading was set at $10 \mu \mathrm{l} / \mathrm{min}$ to produce a theoretical oil mass concentration of approximately $60 \mathrm{mg} / \mathrm{L}$ assuming an oil density of $0.833 \mathrm{~g} / \mathrm{ml}$. To study the effect of dilution of exposure media on droplet size and exposure concentration, a stock dispersion $(60 \mathrm{mg} / \mathrm{L})$ was diluted with artificial saltwater to prepare $20,15,5$ and 2 $\mathrm{mg} / \mathrm{L}$ dispersions. 


\subsubsection{Sample collection}

For the WAF, CEWAF, HEWAF and SHEWAF treatments, samples were sequentially collected at 24 hour intervals during a 96 hour period representative of a typical duration of an acute toxicity testing study. All the treatments were prepared as sets of replicates. For all treatments with the exception of the WAF preparations, two sets of samples were collected for each preparation: a) the original unfiltered dispersion and b) a sample representative of the water soluble fraction (WSF) created by filtering the dispersions containing droplets under little or no negative pressure to prevent loss of volatile compounds using a 4-liter Kontes Ultraware filtration apparatus (Fisher Scientific, Waltham, MA) equipped with a combination of GFD (5 $\mu \mathrm{m}$ pore size) and GFF (0.7 $\mu \mathrm{m}$ pore size) glass microfiber filters in tandem (GE Healthcare, Pittsburg, PA). To improve the retention of droplets and prevent them from reaching the filters, the 1liter filtering holders were packed with about 3 centimeters of Pyrex 3950 fine glass wool (Corning Inc., Corning, NY).

\subsubsection{Droplet characterization using Epi-Fluorescence microscopy}

Samples collected at each time interval were immediately assessed for the presence or absence of droplets using an IX71 Epi-fluorescence inverted microscope (Olympus, Center Valley, PA, U.S.A) fitted with UV epi-fluorescence illumination, a computer-controlled motorized stage (OptiScan, Victoria, Australia), a joystick (Prior Scientific, Rockland, MA, USA), a DP72 digital camera (Olympus, Center Valley, PA, U.S.A) and an advance image analysis software CellSens (Olympus, Center Valley, PA, U.S.A). The UV epi-fluorescence illumination consisted of a band pass excitation filter of 330-385 nm, reflection short filter of $400 \mathrm{~nm}$ and long pass suppression filter of $420 \mathrm{~nm}$. 
The microscope was initially calibrated with a stage micrometer $(0.1 \mathrm{~mm}$ divisions $)$ and the calibration was verified by measuring a series of 6 polystyrene latex beads standards (Polyscience, Inc., Washington, PA, USA) which fluoresced in the blue-violet region of the spectrum $(330-385 \mathrm{~nm})$ with nominal average sizes of $0.97,2.12,4.50,5.40,6.90$ and $10.0 \mu \mathrm{m}$.

Sample preparation for the analysis consisted of depositing a small volume $(5 \mu \mathrm{L})$ of sample on a glass slide containing two $3 \mathrm{~cm}$ chambers with depth of $20 \mu \mathrm{m}$ (Cell-VU DRM-600, Millennium Sciences, New York, NY, USA) and covering the slide with a standard cover slip. The samples were analyzed immediately following collection in order to maintain the integrity of the droplets. For each sample, a total of 15 fluorescence images were captured. The field of view of each image consisted of a size of $874 \mu \mathrm{m} \mathrm{x}$ $658 \mu \mathrm{m}$. Each field of view was selected systematically by moving the microscope slide in two dimensions (positions controlled by motorized stage and joystick) to ensure that no overlapping of fields occurred. The combined frames were statistically representative of the total area surrounding the sample solution in the cover slip. The 15 images were processed in a sequence using the CellSens software and the number and diameter $(\mu \mathrm{m})$ of the droplets were counted and measured and the results were combined. The smallest possible particle dimension detected was $0.64 \mu \mathrm{m}$.

\subsubsection{Droplet size distribution (DSD) analysis using a particle size analyzer (Coulter Counter)}

Droplet size distribution in terms of volume was measured using a Coulter Counter Multisizer 4 (Beckman-Coulter Inc., Miami, Florida). For these experiments, two apertures were utilized with sizes of $30 \mu \mathrm{m}$ and $100 \mu \mathrm{m}$ in order to allow particle 
sizing and counting within the range of $0.6-18 \mu \mathrm{m}$ and $2-60 \mu \mathrm{m}$, respectively. The apertures were initially calibrated with a suspended solution of microsphere standards following the manufacture's recommendations. For all the experiments, $0.2 \mu \mathrm{m}$ filtered artificial saltwater (salinity=33ppt) was used as the electrolyte solution and as a blank control.

Samples were run in triplicate in volumetric mode in order to perform particle concentration analysis. Measurements were made in real-time directly from the freshly prepared sample. To avoid blockage of the aperture, samples that contained high concentration of droplets were pre-diluted by slow addition of electrolyte solution to a predetermined volume of sample in a $20 \mathrm{~mL}$ Accuvette vial. Throughout the analysis, background counts of the electrolyte solution were acquired between samples to ensure the system was not cross-contaminated. The background counts for blanks were significantly low $(<0.01 \%)$ and were not used to correct the particle size distribution data.

Given that all the oil droplets within a treatment prepared with a designated oil had the same oil density, $\rho\left(\mathrm{g} / \mathrm{cm}^{3}\right)$, the total volume of dispersed oil droplets $\left(\mu \mathrm{m}^{3} / \mathrm{ml}\right)$ obtained from the Multisizer 4 software was used to produce a semi-quantitative estimation of oil droplet concentration by applying the following formula:

$$
\text { Oil droplet concentration }(\mu \mathrm{g} / \mathrm{ml})=\left(\mathrm{V} \mu \mathrm{m}^{3} / \mathrm{ml}\right)\left(\rho / 10^{6}\right)
$$

where the factor $10^{6}$ is needed to convert the units of density $\rho$ from $\mathrm{g} / \mathrm{cm}^{3}$ to $\mu \mathrm{g} / \mu \mathrm{m}^{3}$, and to maintain the units of concentration in terms of $\mathrm{ppm}(\mu \mathrm{g} / \mathrm{ml})$. 


\subsubsection{Characterization method for dissolved and entrained oil components in the oil water treatments by Fluorescence excitation emission matrix spectroscopy (EEMs)}

The specific fluorescence of organic compounds in crude oil allows the use of excitation-emission matrix spectroscopy (EEMs) to monitor both dissolved and entrained oil components directly from oil/water samples without any further sample manipulation. The fluorescence measurements were made on a Fluoromax 4 scanning spectrofluorometer (Horiba Jobin Yvon Inc). The excitation and emission slits were both set to $5 \mathrm{~nm}$. A series of automatic emission scans $(300-700 \mathrm{~nm})$ were performed over excitation wavelengths ranging from $250-550 \mathrm{~nm}$ at $5 \mathrm{~nm}$ increments. Contour plots of the EEMs were generated by exporting the data into an Excel spreadsheet to be plotted in Sigmaplot12 (Systat Software, U.S.A). A Quinine sulfate standard was initially used to check the instrument performance and excitation/emission calibration checks were performed daily. Two regions in the spectrum were used to assess dissolved components $\left(\lambda_{\mathrm{ex}}=280 \mathrm{~nm} / \lambda_{\mathrm{em}}=335 \mathrm{~nm}\right)$ versus components in particulate form $\left(\lambda_{\mathrm{ex}}=345 \mathrm{~nm} / \lambda_{\mathrm{em}}=440\right.$ $\mathrm{nm})$.

\subsubsection{Chemical characterization: Total petroleum hydrocarbons by Fluorescence spectroscopy (TPHF)}

Fluorescence spectroscopy was used to calculate total petroleum hydrocarbon concentrations as total oil-related hydrocarbons are correlated to the total fluorescence associated with aromatic compounds in the original test material. The extracts were analyzed in a Horiba Yobin fluoromax-4 scanning spectrofluorometer by measuring the fluorescence intensity at $\lambda_{\mathrm{ex}}=315 \mathrm{~nm} / \lambda_{\mathrm{em}}=415 \mathrm{~nm}$ corresponding to the fluorescence maximum of a Macondo crude oil standard. A linear regression was generated by plotting 
the fluorescence response of solutions of the crude oil standard dissolved in dichloromethane (DCM) in a concentration range from 0.5-50 ppm. Sample dilutions were applied as needed to bring concentrations within the calibrated range. Calculated oil concentration was corrected for volume of sample extract. The method accuracy was assessed by analyzing an oil standard spike that was process with each extraction batch and the recoveries of the extracts were between $85-115 \%$. The statistical method detection limit (MDL) was calculated to be $3.4 \mu \mathrm{g} / \mathrm{L}$ (ppb). The MDL was determined by multiplying the appropriate one sided $99 \%$ t-statistic by the standard deviation obtained from the analysis of seven oil standard spike sample extracts.

\subsubsection{Chemical characterization: Extraction method}

Samples collected for chemical characterization underwent liquid-liquid extraction (LLE) in order to perform quantitation of parent and alkyl PAHs (PAH50) by GC-MS analysis and TPHF analysis (described in section 2.2.9) from each individual treatment at time $0,24,48$ and 96 hours in order to evaluate the decay of overall droplet and oil concentrations and to assess changes in the fine chemical composition with time. Water samples (200-300 mL) were processed by using a separatory funnel equipped with a Teflon cap and a stopper. For the PAH extraction, all the samples were amended with PAH surrogate standards (d8-naphthalene, d10-acetanaphene, d10-phenanthrene, and d12-perylene) and extracted three times using $50 \mathrm{~mL}$ of DCM. The walls of the glass bottles containing the sample were rinsed with DCM to assure the transfer of all the analytes into the separatory funnel. The organic layers were combined, dried using combusted anhydrous granular sodium sulfate and collected in a $250 \mathrm{~mL}$ flat-bottom 
round flask. The extracts were evaporated to about $10 \mathrm{~mL}$ in a water bath at $56^{\circ} \mathrm{C}$, transferred to concentration tubes and concentrated down to $1 \mathrm{~mL}$ under a gentle stream of clean nitrogen. PAH internal standards (d10-fluorene and d12-benzo(a)pyrene) were added to the concentrated extracts before analysis in order to determine the recovery of the surrogate standards. Water samples extracted for TPHF analysis were processed in a similar way as PAHs without the addition of surrogates or internal standards (due to interfere in the detection) and concentrated down to a final volume of $5 \mathrm{~mL}$.

\subsubsection{GC-MS analysis}

Sample extracts were analyzed using a Thermo Scientific Ultra Trace TSQ Quantum XLC gas chromatography-triple quadrupole mass spectrometry (GC-QqQMS) fitted with a DB-5MS fused silica capillary column (30 m with $0.25 \mu \mathrm{m}$ film thickness and $0.25 \mathrm{~mm}$ I.D.). Detection of PAHs was done in electron impact mode $(70 \mathrm{eV})$ operated using selected ion monitoring (SIM) mode. The sample extracts $(2 \mu 1)$ were injected into a $300{ }^{\circ} \mathrm{C}$ split/splitless injector operated in splitless mode. The helium flow rate was held constant at $1.7 \mathrm{ml} / \mathrm{min}$ and the $\mathrm{GC}$ oven was programmed from $40{ }^{\circ} \mathrm{C}(1$ min hold) at a rate of $7.5^{\circ} \mathrm{C} / \mathrm{min}$ to $295^{\circ} \mathrm{C}(8 \mathrm{~min}$ hold $)$.

\subsection{Results and Discussion}

\subsubsection{Droplet characterization}

The physical characterization of both unweathered MASS oil and one of the weathered oils (CTC) are shown in Figure 2 and 3, respectively. The results for both oils, MASS and CTC, based on the microscope images show that droplets were not optically detected in the low energy WAF preparations (Fig.2 and Fig.3, top). For the MASS oil, 
with the exception of the low energy WAFs, all preparations for MASS oil contained droplets within sizes ranging from $0.64-16 \mu \mathrm{m}$ in diameter. The only significant differences among MASS preparations containing droplets is that the majority of droplets in the CEWAF preparations were less than $2 \mu \mathrm{m}$, while most of the droplets formed in the SHE/HEWAF preparations were larger than $2.5 \mu \mathrm{m}$ (Fig.2, top).

In addition, based on the histograms obtained from the Coulter Counter analysis which show the DSD in terms of volume $\left(\mu \mathrm{m}^{3}\right.$ per $\mathrm{mL}$ of sample) it can be seen that there is an increase in the material introduced into the water with increase in mixing energy (CEWAF $<$ HEWAF $<$ SHEWAF, Fig.2, bottom). As a result of the low dispersibility of the weathered or more viscous oils, the CEWAF preparations using CTC were not effective and droplet formation was limited as shown in the microscope image (see Fig. 3, top). On the other hand, high energy preparations successfully generated droplets with size distributions centered at $\sim 2.5 \mu \mathrm{m}$ (Fig.3) and a general increase in the entrained oil with increase of mixing time was observed.

Overall, the droplet sizes generated in all the preparations were smaller than the size ranges reported or described by models as potentially present during the DWH incident (10-50 $\mu \mathrm{m})$ (Li et al., 2011; Yapa et al., 2012). However, the droplets formed in these particular preparations are still important to study as droplets smaller than about 3 $\mu \mathrm{m}$ will remain suspended in the water column for extended periods of time and may be associated to important environmental processes (McAuliffe et al., 1980; North et al., 2011). Small droplets can further influence the stability and composition of the exposure media used for toxicology testing. 
WAF
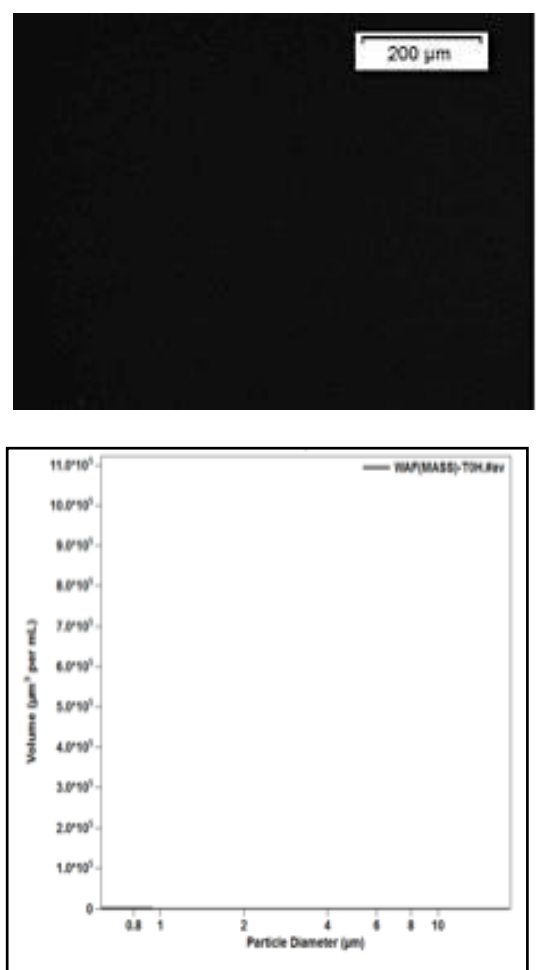

CEWAF
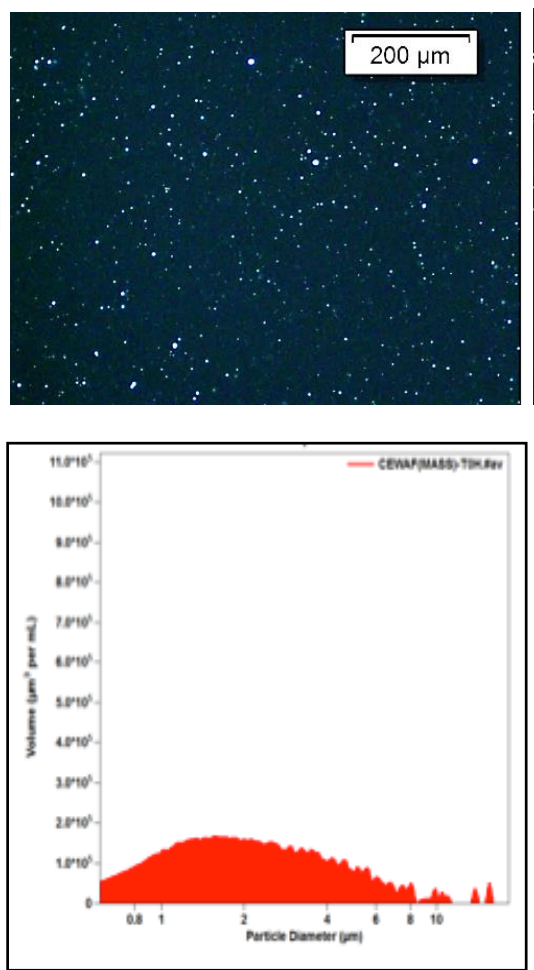

HEWAF
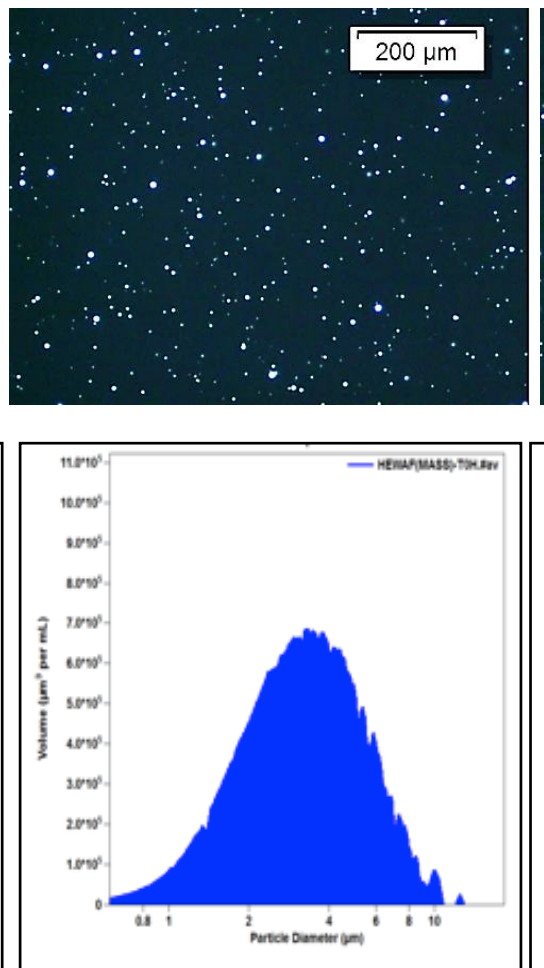

SHEWAF
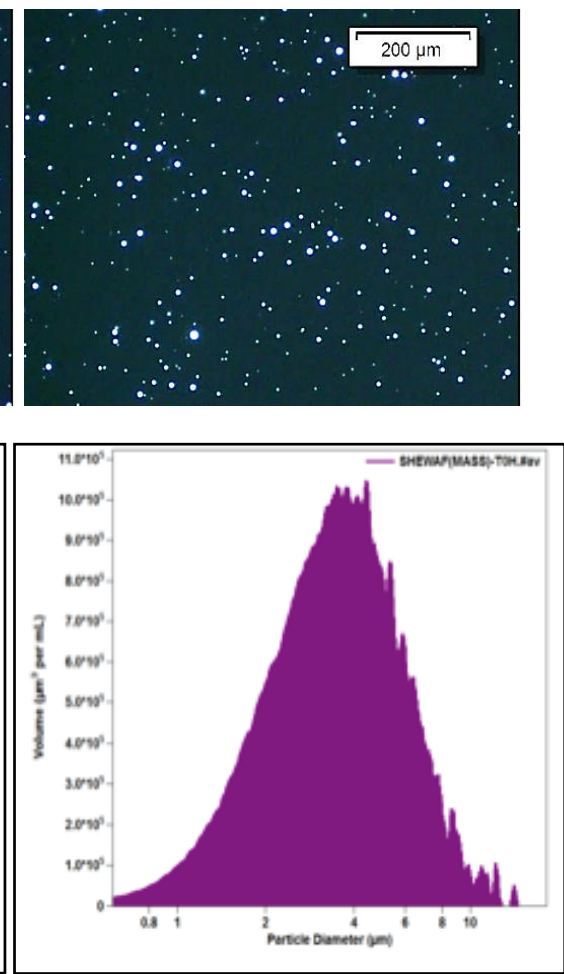

Figure 2: Physical characterization of different treatments (water accomodated fractions (WAF), chemically enhanced water accomodated fractions (CEWAF), high energy water accomodated fractions (HEWAF) and super high energy water accomodated fractions (SHEWAF)) prepared with MASS oil, Epi-fluorescence microscope images (top) and Coulter Counter histograms (bottom) measured at initial preparation time after settling. 
WAF
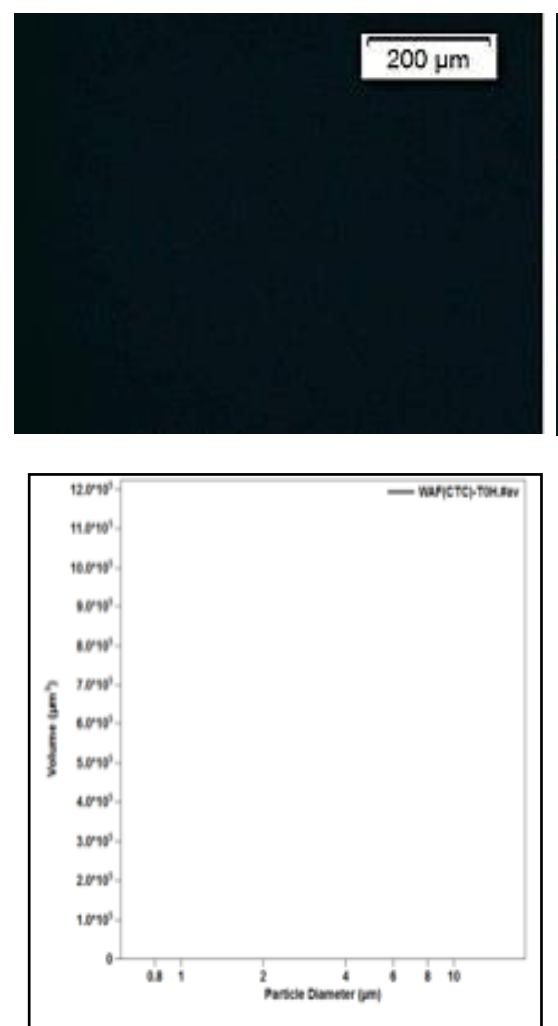

CEWAF
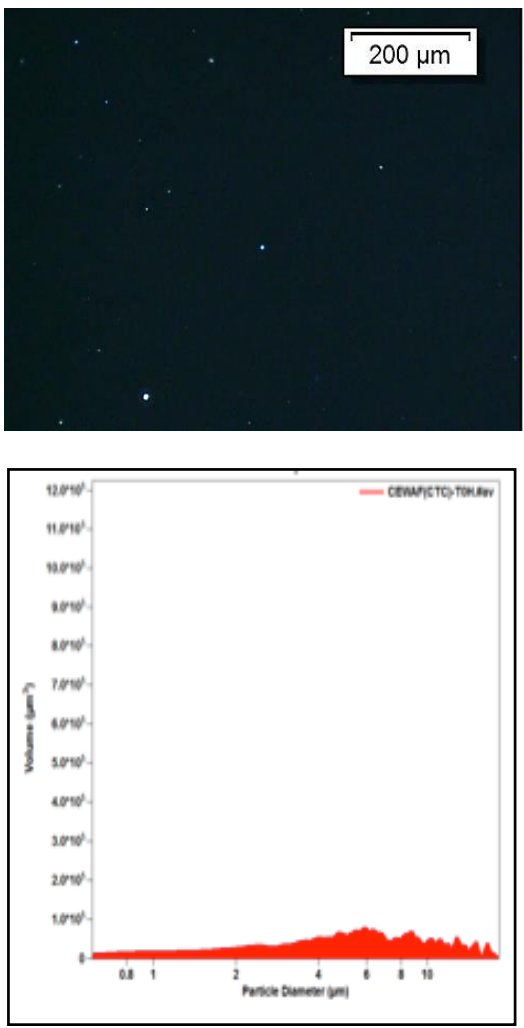

HEWAF
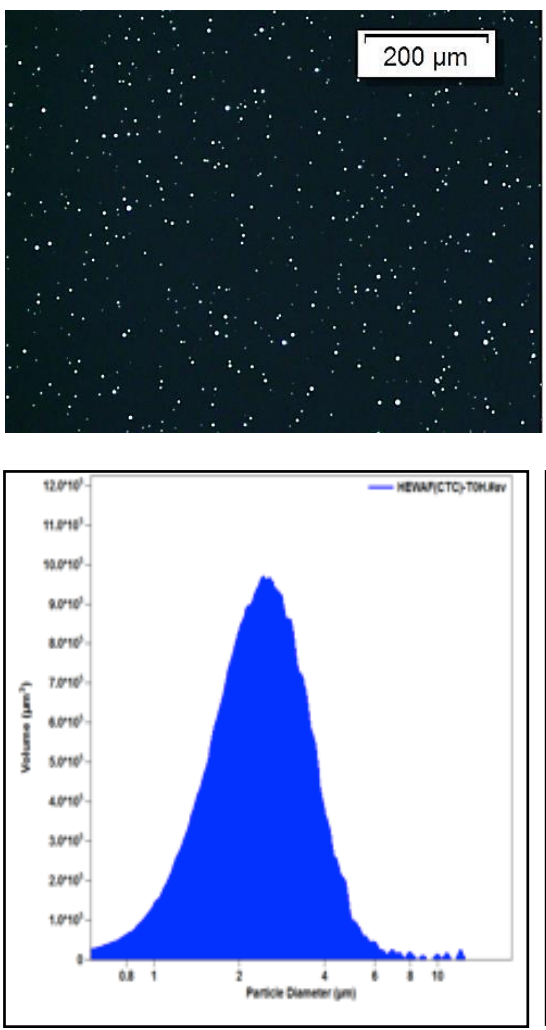

SHEWAF
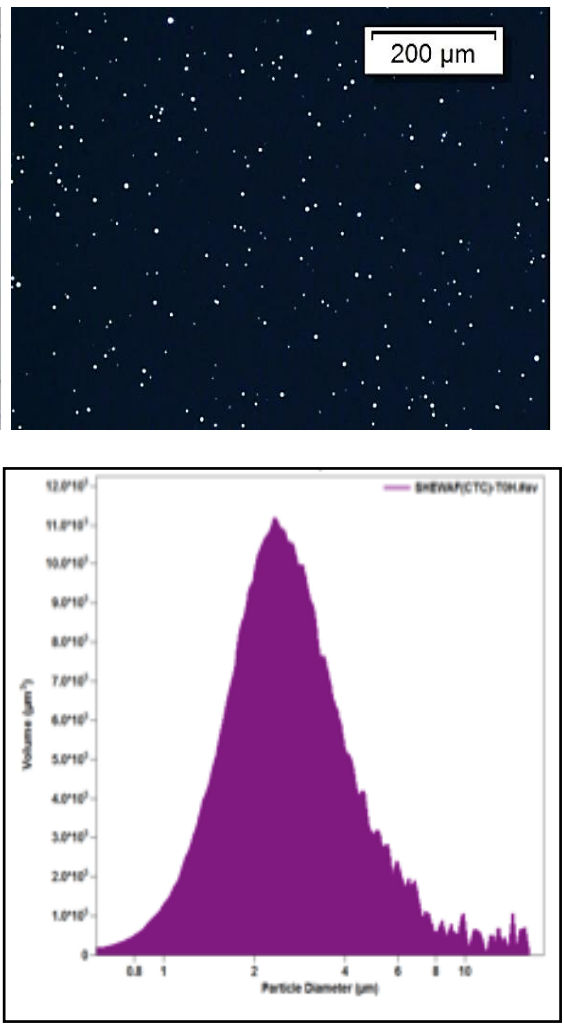

Figure 3: Physical characterization of different treatments(water accomodated fractions (WAF), chemically enhanced water accomodated fractions (CEWAF), high energy water accomodated fractions (HEWAF) and super high energy water accomodated fractions (SHEWAF)) prepared with CTC oil, Epi-fluorescence microscope images (top) and Coulter Counter histograms (bottom) measured at initial preparation time after settling. 


\subsubsection{Physical characterization of dissolved versus droplet phase by Fluorescence spectroscopy}

The EEMs were acquired to monitor the dissolved and entrained oil components directly from the oil-water preparations. An example of the EEMs expressed as contour plots for a WAF and CEWAF preparation prepared with the MASS oil is shown in Figure 4. There is a clear difference between the preparation that contain the oil droplets (CEWAF) and the one that is composed mainly of the dissolved components of the oil (WAF). The addition of dispersant which resulted in the generation of small droplets produced high fluorescence intensity signals over an emission range of 400-550 nm throughout the range of excitation wavelengths. The WAF preparation exhibit a fluorescence maxima intensity at $\lambda_{\mathrm{em}}=335 \mathrm{~nm}$ over an excitation range of $280-290 \mathrm{~nm}$.

The CEWAF preparation also displayed the same fluorescence characteristic though it is obstructed by the magnitude of the other fluorescence signals. These observations are consistent with the results obtained by Bugden et al. (2008) who showed that various crude oils dispersed with Corexit $^{\circledR}$ EC9500a exhibit a large increase in fluorescence intensity at $\lambda_{\mathrm{em}}=445 \mathrm{~nm}$ and all the crude oils tested also exhibited an emission signal at $340 \mathrm{~nm}$ over an excitation range of 280-300 nm. Furthermore, EEMs for a chemical dispersion prepared with Macondo oil and Corexit ${ }^{\circledR}$ EC9500a also displayed similar fluorescence signal patterns (Zhou et al., 2013). Bugden et al. (2008) and others have attributed the emission peaks at $340 \mathrm{~nm}$ to be a characteristic of the more water soluble low and medium molecular weight aromatics, while the emission peak at $445 \mathrm{~nm}$ is probably associated with the higher molecular weight ( $\geq 3$-ring) aromatics. 
From the EEMs, it can be infer that the fluorescence signal observed at a maximum $\lambda_{\mathrm{ex}}=280 \mathrm{~nm} / \lambda_{\mathrm{em}}=335 \mathrm{~nm}$ in the WAF and CEWAF preparations results from the soluble components of the oil; while the signal observed at a maximum $\lambda_{\mathrm{ex}}=345$ $\mathrm{nm} / \lambda_{\mathrm{em}}=440 \mathrm{~nm}$ is due to the presence of droplets generated by the addition of dispersant in the CEWAF preparations. The data obtained from the contour plots show a simple way to distinguish between dispersed oil (with droplets) and dissolved fractions (without droplets), but do not provide a direct way to measure the specific aromatic components within the area of fluorescence as factors such as physical (viscosity and optical density) and chemical composition (concentration of fluorophores and quenching agents) can all lead to a variation in emission wavelengths and intensity. However, the use of EEMs is a rapid and cost-effective method to monitor chemically or mechanically dispersed oil in seawater and to verify the presence of droplets in unprocessed and un-extracted water samples.

The fluorescence of natural CDOM can interfere with the fluorescence of crude oil components; however there are possible ways to distinguish the different signals. Collecting fluorescence data over a wide range of ex/em wavelengths can help to determine the contributing source of other fluorescence signals. For example, Zhou et al. (2013) was able to identify four DOM fluorescence components by using PARAFAC modeling from the EEM data collected from seawater samples from the GOM. The use of modeling tools can further enhance the use of EEMs in order to characterize oil components in seawater samples in real environmental settings. 

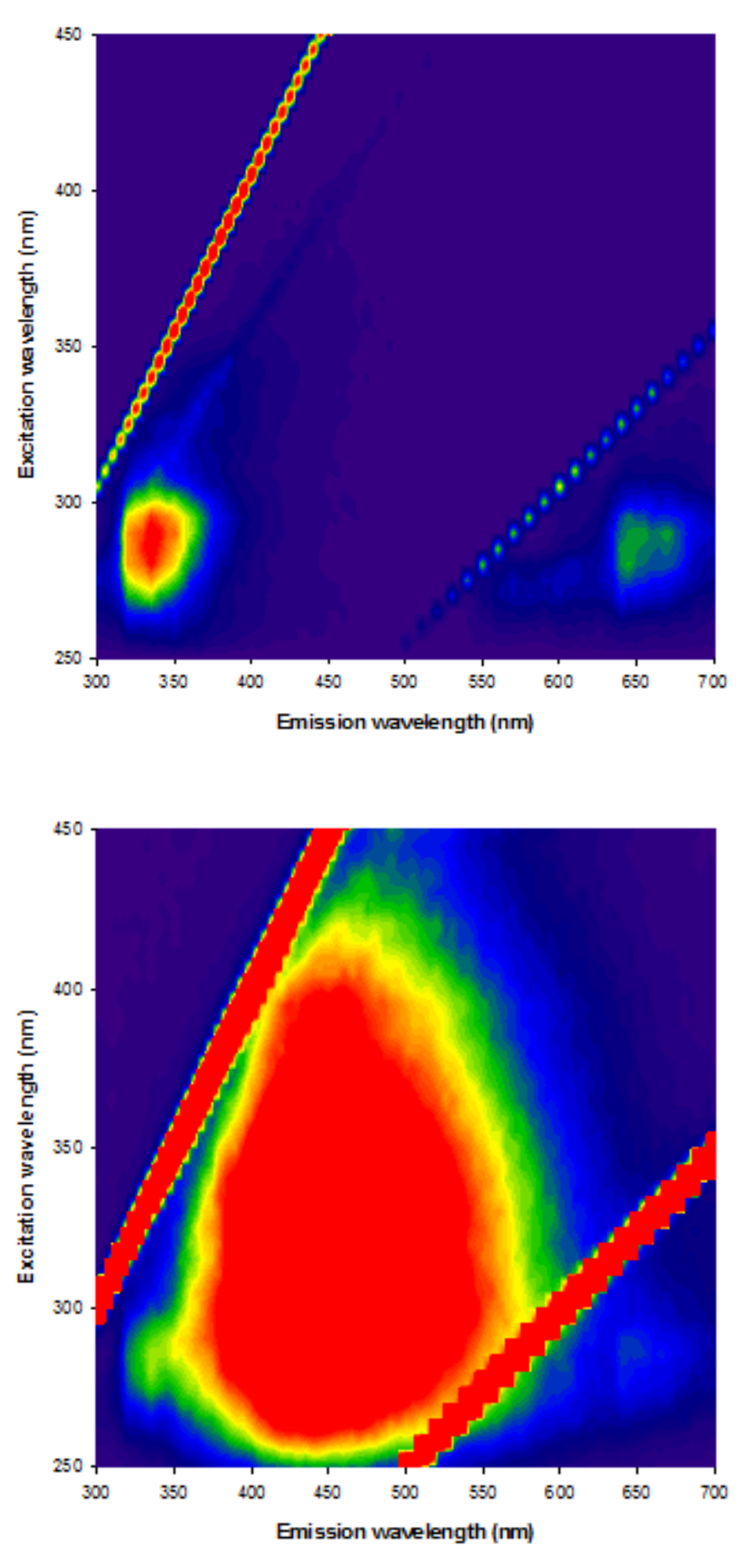

Figure 4: Fluorescence excitation emission matrix (EEM) spectra showed as contour plots for the water accommodated fraction (WAF) (top) and the chemically enhanced water accommodated fraction (CEWAF) (bottom) preparations using the source MASS oil. The red diagonal lines across the EEMs correspond to the Rayleigh and Raman scattering peaks. 


\subsubsection{Effects of weathering on physical characteristics of the oil-water mixtures}

The influence of oil weathering on droplet sizes was studied using only the high energy preparations because they were capable of producing droplets for all materials tested. As oil weathers, it loses its soluble components, gets stripped of volatile components and is subject to biodegradation. Through all these processes it becomes more viscous and thus the specific density of the oil increases significantly. The weathering order for the oils tested is as followed: MASS (specific gravity of 0.84), CTC (specific gravity of 0.98 ) and Juniper (specific gravity of 1.00).

The average volume median diameter (VMD) for the three oils for both SHE/HEWAF preparations was plotted against the oils specific density and shown in Figure 5. All treatments were generated with the same oil loading $(1 \mathrm{~g} / \mathrm{L})$ and similar settling conditions such that the only differences were the time of mixing ( 30 seconds for HEWAF, 120 seconds for SHEWAF) and the test material itself. The results show that the more weathered oils produced dispersions with significantly smaller droplet sizes than the dispersions prepared with the source oil. In addition, droplet sizes were slightly larger in the SHEWAF preparations as opposed to the HEWAF preparations suggesting that the higher droplet density created in the SHEWAFs could be more conducive to coalescence. Experiments conducted with Arabian crude oils at slower mixing speeds ( $10000 \mathrm{rpm})$ showed similar patterns with an increase in both droplet diameters and droplet count with increase in mixing time (Less and Vilagines, 2013). That study also concluded that such effects are dependent on crude oil type and oil viscosity.

These findings are important to take into account when high energy preparations are used as the size of droplets will affect both the chemical composition and stability of 
the exposure media. The use of high energy of mixing does have the intrinsic advantage of producing droplets from weathered oils that could not be dispersed under normal conditions or with the addition of chemical dispersants, however, their use in toxicological studies also brings to question whether the mixing energy used in these SHEWAF/HEWAF preparations can be related to realistic wind and wave conditions in open-ocean or shoreline scenarios.

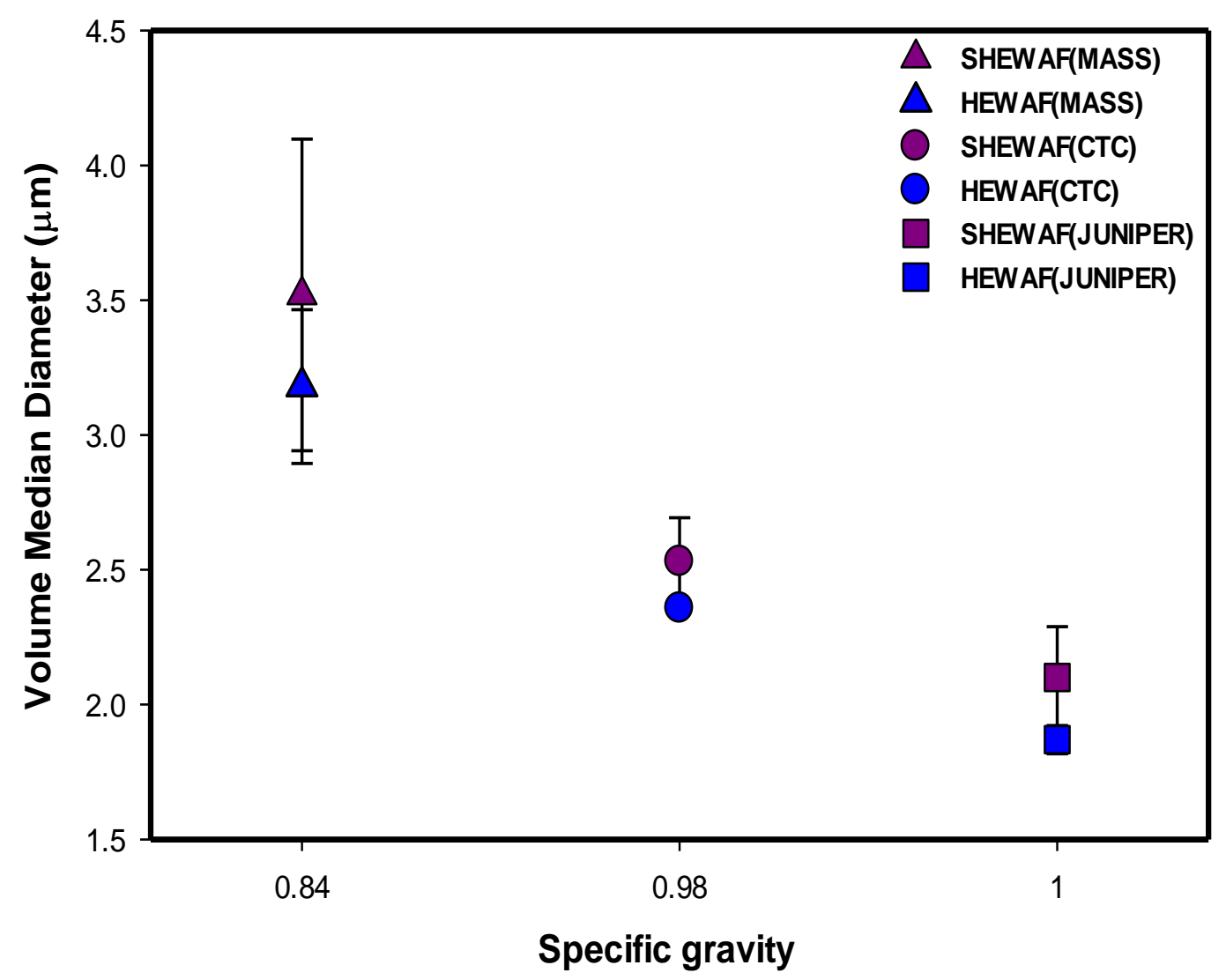

Figure 5: Influence of oil weathering in terms of specific gravity (MASS $=0.84$, $\mathrm{CTC}=0.98$, and Juniper $=1.00)$ on the volume median diameter $(\mu \mathrm{m})$ of droplets measured by Coulter Counter for the high energy preparations.

To date the effects of small oil droplets on overall toxicity to marine organisms is not fully understood. Some studies have found that oil droplets can be ingested or coat 
body appendages, however, the bulk of the available literature suggest that the main toxicological effects results primarily from exposure to the dissolved oil components (Bejarano et al., 2014). However, without proper characterization of droplet sizes, the stability of the droplets during the duration of the studies, and the chemical composition of both the soluble and insoluble compounds present in the exposure media, the actual toxic effects contributed by oil droplets cannot be fully described.

Franklin and Lloyd (1986) suggested that a relationship with droplet size and toxicity of dispersant/oil mixtures is conceivable as a reduction in droplet size will lead the organisms to come into contact with a greater surface area of oil in which toxic components can diffuse. Alternatively, the formation of smaller droplets (resulting in larger surface areas) will accelerate the dissolution of oil components but their final concentrations is still dominated by their solubility (Franklin and Lloyd, 1986). Bobra et al. (1989) suggested that larger droplet sizes do cause a decrease in toxicity but they also stated that the relationship between droplet size and effects is difficult to establish.

In addition, the bioaccumulation of petroleum hydrocarbons in crustaceans has been suggested to be dependent on the characteristics of oil dispersions specifically small droplet sizes (Chase et al., 2013) but modeling the contribution of droplets to bioaccumulation of oil components also resulted in negligible changes when compared to the accumulation from the dissolved phase components (Viaene et al., 2014). The result of all these differing evidence clearly points out that a full physical and chemical characterization of oil-water dispersions is needed on every study to isolate physical and chemical effects when establishing potential links between the oil present in the exposure media and the observed biological reposes. 


\subsubsection{Effects of oil loading on droplet size for high energy preparations}

Toxicity test results can be affected by multiple factors involving the parameters used in the exposure media preparation. A key one is the initial oil loading; a starting point is to have enough oil to reach the maximum possible concentration of the soluble components, often called a functional equilibrium. Earlier fundamental work presented in Singer et al. (2000) recognized that preparation of non-vortex WAFs at oil-to-water loading ratios from as high as $1 / 100(10 \mathrm{~g} / \mathrm{L})$ to $1 / 100,000(10 \mathrm{mg} / \mathrm{L})$ did not result in significant differences in the GC/FID traces. In addition no insoluble material was present as indicated by the absence of identifiable n-alkane signatures or fluorescence microscopy (Singer et al., 2000). However, when higher mixing energies are used, the exposure media tends to chemically reflect the composition of the entrained bulk oil. While preparing SHEWAF/HEWAF dispersions with both fresh and weathered oils, observations made in this study indicated that even at loadings of 1/1000 oil-to-water ratio, a lot of the test material was left on the walls of the container. Because multiple reports in the literature have suggested the use of different loadings when preparing high energy mixtures (Incardona et al., 2014; Mager et al., 2014), an evaluation on the effects of loading rates and sequential dilution on the physical and chemical properties of the exposure media was conducted.

The results were similar for both SHEWAF/HEWAF preparations and more pronounced in dispersions containing the weathered oils so a representative example of a SHEWAF using CTC oil is shown in Figure 6. The first observation, presented on the top graph in Figure 6 shows that droplet sizes changed significantly with oil loading showing a twofold decrease in the average sizes when loadings decreased from $1000 \mathrm{ppm}$ to 20 
ppm $(2.53$ to $1.07 \mu \mathrm{m})$. In complete contrast, however, when a $1000 \mathrm{ppm}$ dispersion is sequentially diluted over the same concentration ranges the droplet size (VMD) remains relatively constant $($ Average $=2.59 \mu \mathrm{m} \pm 5.2 \%$, range $=2.53$ to $2.40 \mu \mathrm{m})$.
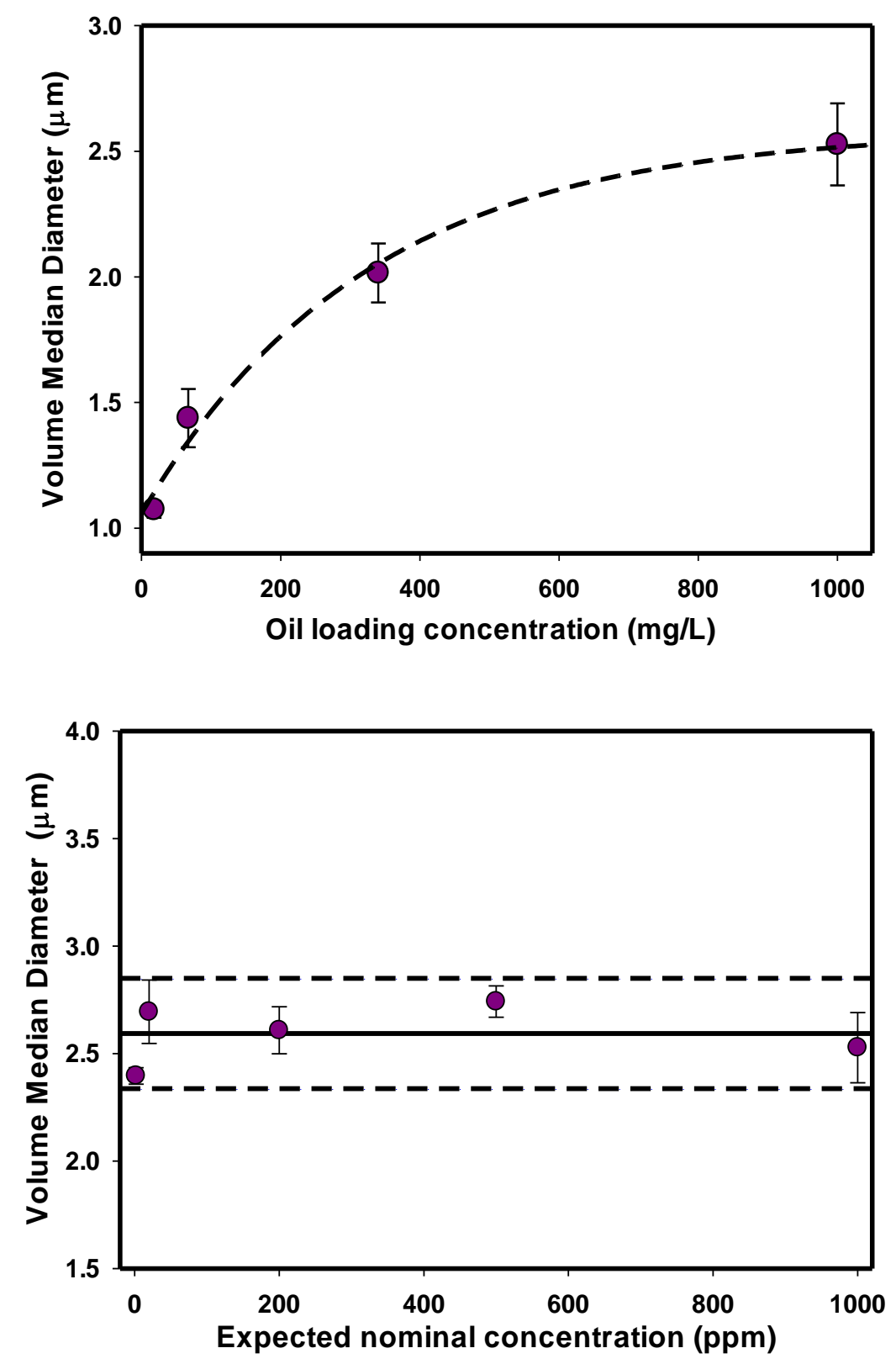

Figure 6: Volume median diameter $(\mu \mathrm{m})$ measured by Coulter Counter for different nominal oil loadings (top) and dilution series of a $1000 \mathrm{ppm}$ stock solution (bottom) for SHEWAFs prepared with CTC oil. 
Because potential discrepancies between the nominal loadings and the actual concentrations could be significant, the data were re-plotted using the bulk oil concentrations measured by both fluorescence spectroscopy (TPHF) and Coulter Counter. Figure 7 shows the relationships between nominal and measured concentrations for the SHEWAF preparations with CTC oil and the corresponding trends with droplet sizes. As expected, a non-linear relationship exists between nominal loading and the measured (TPHF) or inferred oil concentrations obtained from the Coulter Counter. This result indicates that the excess oil in higher loading preparations is not introduced into the dispersion and likely stays in the mixing container or the separatory funnels used for the settling.

When the droplet size distributions are plotted against the measured, rather than nominal values, the relationship between decreasing loadings and decreasing droplet sizes becomes linear $\left(r^{2}>0.98\right)$ as shown by the two complementary but independent bulk oil determinations, where TPHF measurements are shown in red and Coulter Counter measurements are shown in blue (Fig.7, top). This behavior was observed for both weathered oils tested in the high-energy preparations. These findings clearly indicate that both the weathering stage and the initial oil loading do control the droplet size formation in preparations that use extensive mixing to produce droplets. Because a change in droplet size could alter the way oil interacts with specific receptors, full characterization of these solutions are needed to eliminate hidden confounding factors influencing the determination of effects. 

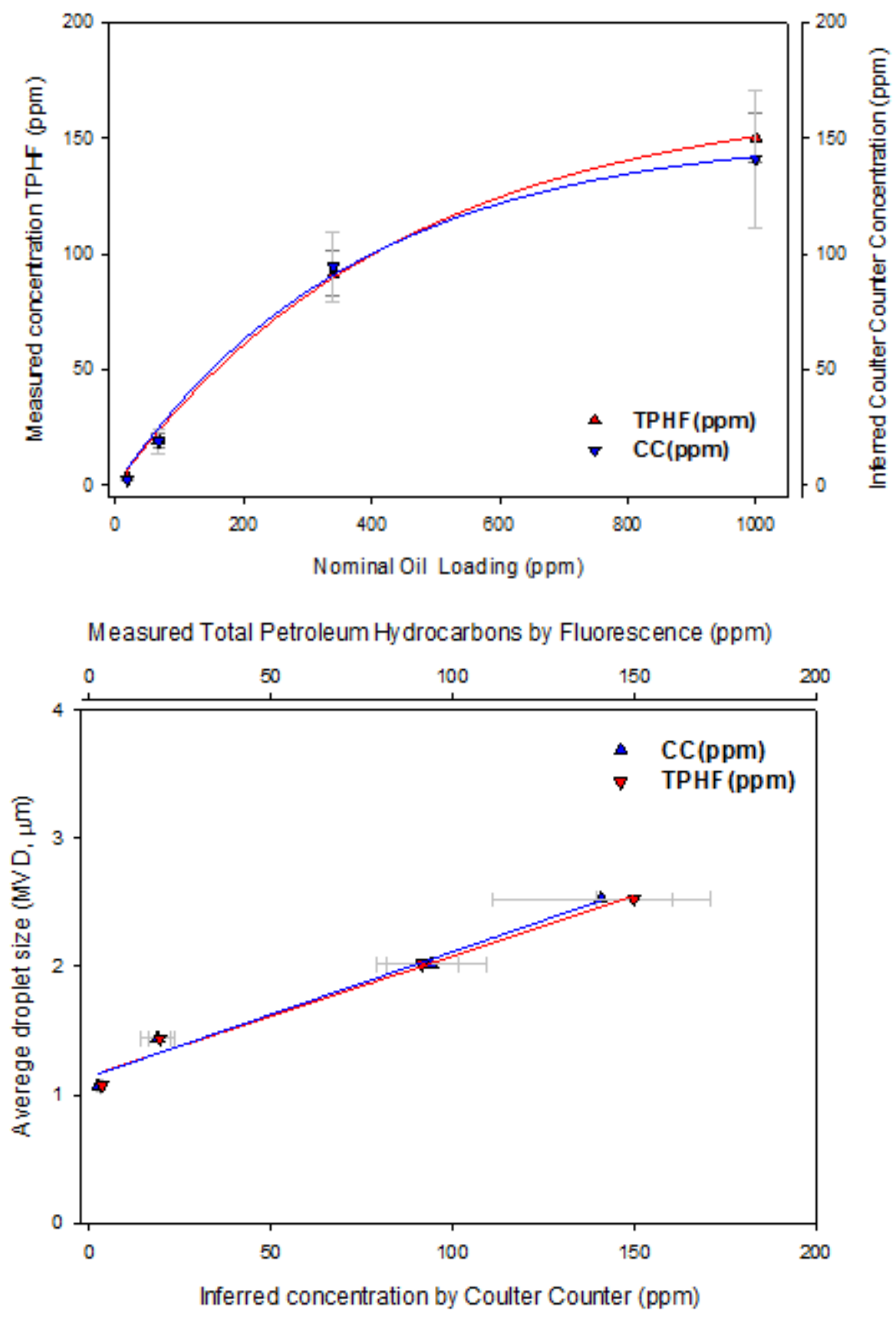

Figure 7: Relationship between nominal and measured concentrations (top) where TPHF measurements are shown in red and Coulter Counter measurements are shown in blue for SHEWAFs prepared with CTC oil and the corresponding trends with droplet sizes (bottom) showing a linear dependency between decreasing measured loading concentrations and particle size distributions. 


\subsubsection{Dilution series of oil water dispersions (OWDs) prepared with MASS oil}

The effect of dilution on droplet sizes and droplet concentrations were further explored for OWDs prepared with MASS oil using the droplet generator method described by Nordtug et al. (2011a) which allows the preparation of exposure media with consistent droplet sizes and reproducible concentrations. The DSDs of the dilution series obtained from the Coulter Counter analysis (Fig.8, top) show consistent size distributions following a uniform distribution centered at a diameter size of $11.98 \mu \mathrm{m}$. These results were consistent with previous experiments which generated droplets of similar sizes using the droplet generator system (Nordtug et al., 2011a; Nordtug et al., 2011b). Unlike the high energy preparations, the nominal concentrations of the dilution series and the actual concentrations measured by both the Coulter Counter and the fluorescence method were consistent (Fig.8, bottom).

Furthermore, the validity of both the Coulter Counter and fluorescence method for the determination of the bulk oil concentration was demonstrated as the measured concentrations obtained from both methods were consistent with the nominal loading concentrations $\left(\mathrm{r}^{2}>0.99\right)$. The Coulter Counter method proved to be a reliable method to determine the bulk oil concentration based on dispersed oil droplets in real time with the same accuracy as the fluorescence method which involved extensive wet chemistry. It should be noted that measurements acquired with the Coulter Counter should be performed immediately after sample collection as settling time can lead to resurfacing of oil droplets. These results indicate that dilutions of exposure media do not affect the physical composition of the exposure media for the OWDs and are consistent with the results shown in the previous section. 

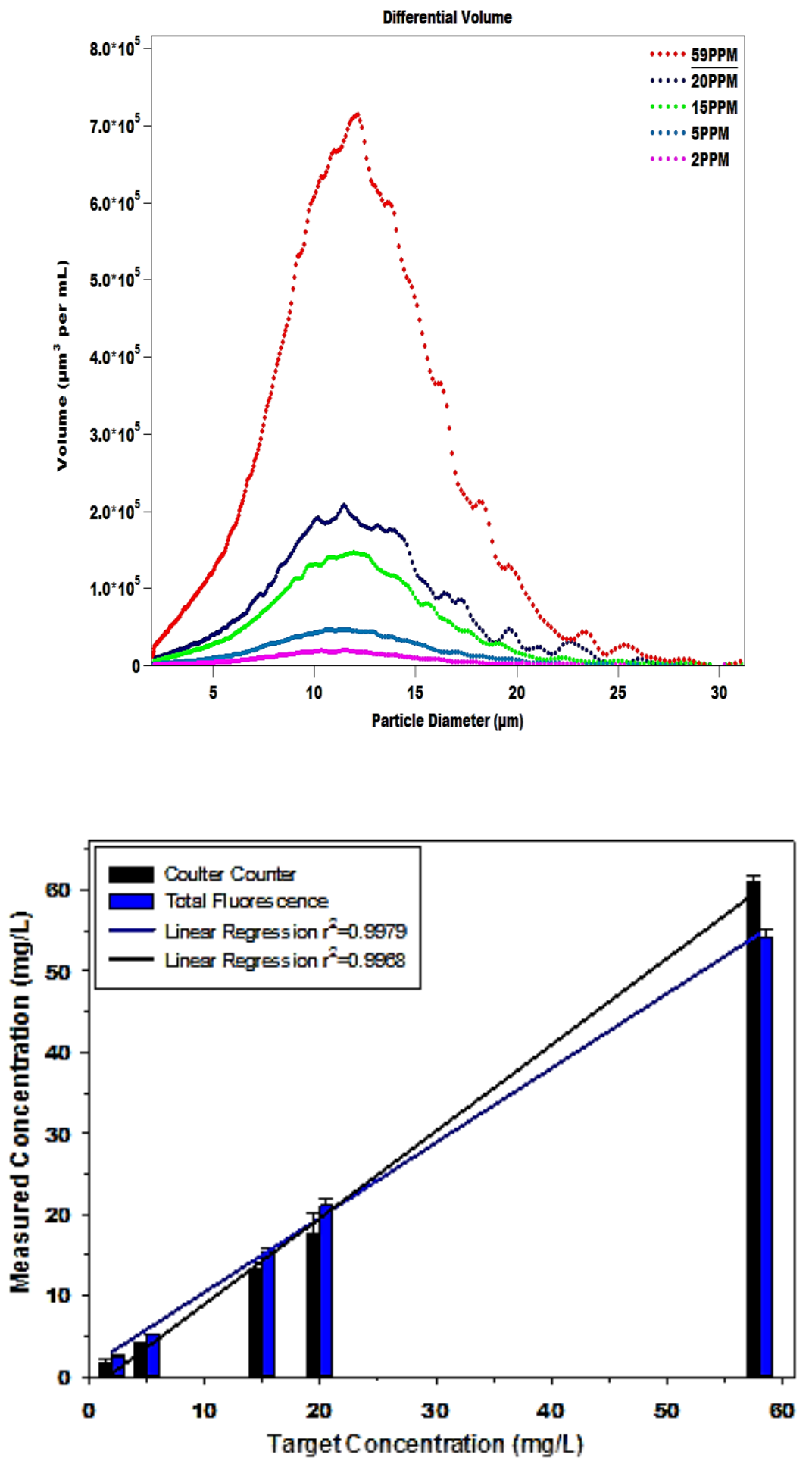

Figure 8: Droplet size distributions (based on volume) obtained from Coulter Counter analysis for the dilution series of OWDs $(2,5,15,20,60 \mathrm{mg} / \mathrm{L}$, top). Comparison of oil concentration measurements by Coulter Counter and total petroleum hydrocarbons by fluorescence of the dilution series (bottom). 


\subsubsection{Stability of different oil loading preparations with time}

The last set of results describes the differences in dispersion composition with respect to the test material and the mixing protocols at the start of an experiment. The next section concentrates on their evolution during a typical 96 hour period relevant to acute toxicity studies. Figure 9 shows the changes in the bulk composition of the exposure media, expressed as the TPHF in $\mathrm{mg} / \mathrm{L}$, for the multiple loading series for the SHEWAF preparations with the CTC oil described before, as a function of time. The most apparent difference is observed in the 1000 ppm loading which showed an exponential decrease in concentration in the first 24 hours with approximately $94 \%$ loss in total petroleum concentration at which point steady state was reached.

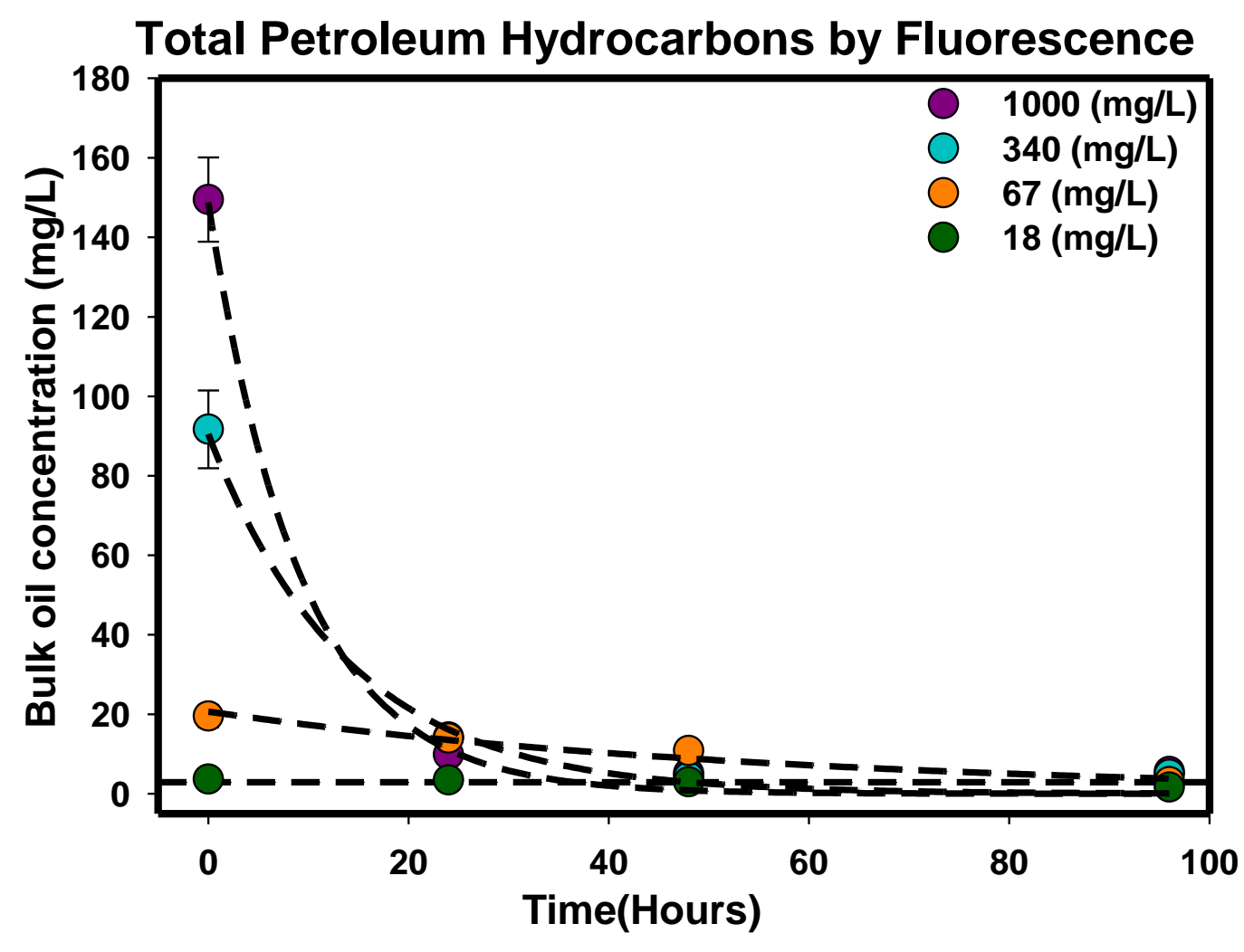

Figure 9: Stability of the SHEWAF preparations with CTC oil at different oil loadings measured as TPHF over a 96 hour period. 
The stability of the dispersion improved as the oil loading decreased with an $87 \%$, $27 \%$ and $5.1 \%$ loss of total petroleum concentration in the first 24 hours for the 340,67 , $18 \mathrm{mg} / \mathrm{L}$ oil loading, respectively. These results suggest that most of the variability in concentration occurs in the first 24 hours as concentrations measured after 24 hours were mostly consistent. The discrepancy in stability is most likely a result of the difference in droplet size distribution, as smaller droplets are more stable and will remain in solution for longer time. Higher oil loadings results in the introduction of more and larger droplets which will coalescence to increase the fraction of dispersed oil that will resurface during longer settling periods or while in a test chamber.

Droplet sizes would not only determine the introduction rate and permanence of the bulk oil in the water column but will also dominate the chemical composition of the exposure media. To date several publications have used the HEWAF preparations to conduct toxicity (Incardona et al., 2013; Brewton et al., 2013; Incardona et al., 2014; Mager et al., 2014). One commonality on all these reports is the use of the commercial blender that allows the preparation of larger volumes of dispersions in a systematic fashion using weathered oils; however, there are also significant differences in the loadings (from not reported to $50 \mathrm{mg} / \mathrm{L}$ to $1 \mathrm{~g} / \mathrm{L}$ ), the preparation protocols (30 seconds and 2 minute mixing times) and important differences in the times where samples were taken for analysis with respect to their introduction to the testing containers (from immediately after loading to the separatory funnel to 1 hour after settling). As shown, all of these factors affect the composition of the exposure media. 


\subsubsection{Chemical characterization of exposure media preparations}

The PAHs and alkyl PAHs were measured in all 1:1000 dispersions at initial collection time after the appropriate settling time (T0) for all treatments with MASS, CTC and Juniper oils and the results are shown in Figure 10 with the same concentration scale to allow comparison among them. Because of the relatively higher abundance of the 2-ring aromatics (naphthalenes), they are shown in a separate area with an expanded scale for the MASS and CTC oil, while the Juniper oil is shown at a scale that is 8 times smaller. The WAFs are constituted entirely of soluble components and the differences seen in the PAH chemistry are due to the weathering or the initial material, the naphthalenes dominate the WAF compositions prepared with the MASS oil, while the WAFs prepared with CTC and Juniper oil are composed mainly of the 3-ring PAHs (fluorenes, dibenzothiophenes and phenenthrenes).

Based on the distributions of PAHs, the high-energy preparations resulted in dispersions with elevated concentrations of entrained oil with characteristics that resemble the original test material. For the MASS oil, SHEWAF and HEWAF preparations introduced similar amounts of PAHs in the dispersions. However, for the weathered oils an increase in energy resulted in an increase in the amount of PAHs (as particulate oil) introduced in the dispersions. Both high-energy methods (for the MASS oil) resulted in PAH concentrations that are four times higher than the CEWAF preparation and 15 times higher than WAF preparation. The behavior of oils in the CEWAF preparations were in accordance with their dispersability and the TPAH concentrations reflected that. These findings corroborate the results obtained from the physical characterization. 

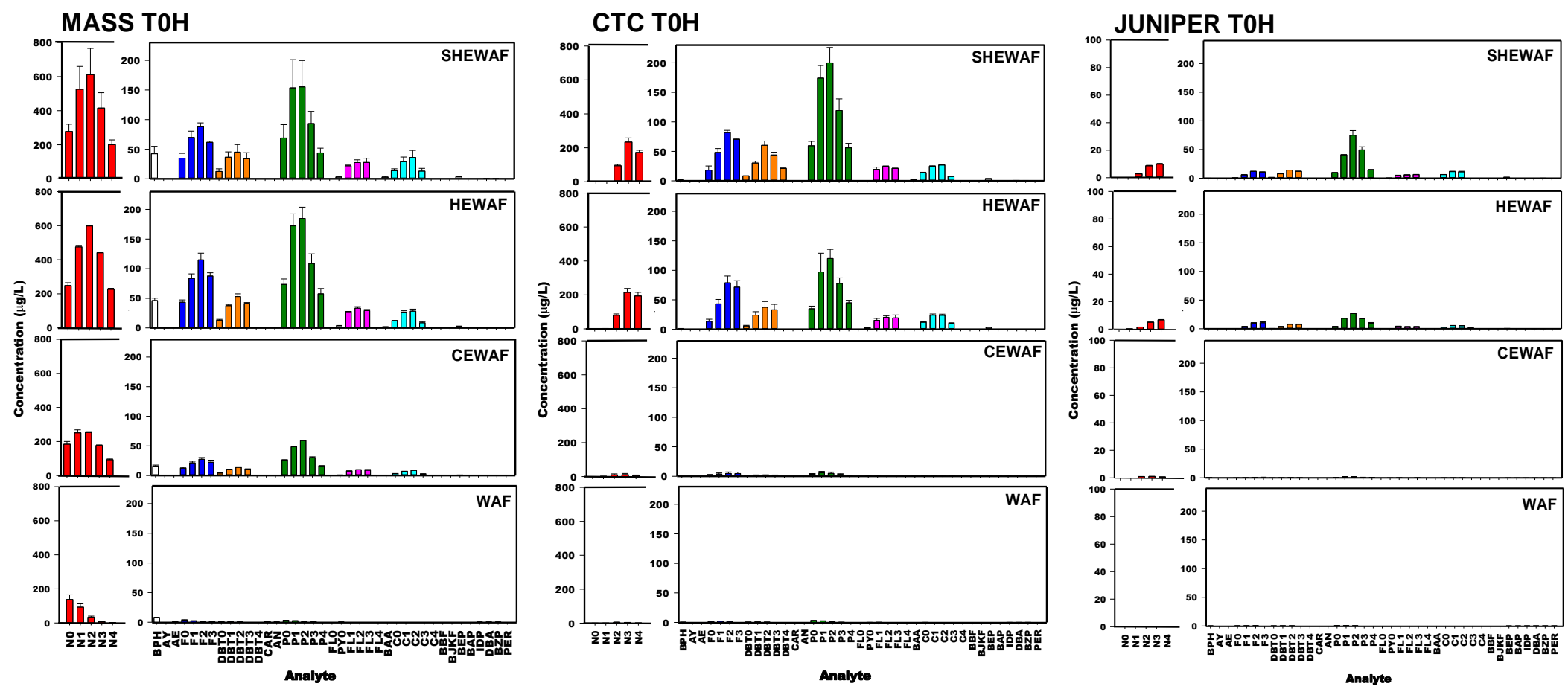

Figure 10: PAH profiles and concentrations for MASS (left) CTC (middle) and JUNIPER (right) oils preparations at initial collection time for all treatments (water accomodated fractions (WAF), chemically enhanced water accomodated fractions (CEWAF), high energy water accomodated fractions (HEWAF) and super high energy water accomodated fractions (SHEWAF)). Abbreviations for PAHs are described in appendix 1. 


\subsubsection{Chemical stability of oil preparations with time}

The stability of the different preparations for all oils was further examined over a 96 hour period relevant to an acute toxicity test. Stability was determined as TPAHs losses over time. The concentrations of TPAHs for the WAF preparations were consistent with the concentration of soluble components in the test material and stable over time for the three oils, while high-energy preparations were highly dynamic and subject to considerable oil resurfacing within few hours from initial preparation (Fig.11). Most of the changes in chemical composition occurred during the first 24 hours after initial preparation. Table 1 shows the $\%$ TPAHs losses during the first 24 hours for the different treatments and oils. The WAF and CEWAF preparations were the most stable preparations with less than $33 \%$ loss for all oils. On the other hand, in the case of the high energy preparations up to $90 \%$ of the TPAHs were lost within the first 24 hours and the dispersion stability was dependent on the test material weathering stage.

The increase in stability by increase in weathering stage for the high energy preparations could be explained by the sizes of the droplets formed in the high-energy treatments. Smaller droplets are known to be more stable and remain in solution; therefore the TPAH concentrations were not as dynamic for the more weathered oil (Juniper) which contained smaller droplets in comparison to the unweathered MASS oil. The larger droplets formed in the SHE/HEWAF preparations for MASS and to a lesser extent CTC oil most likely resurfaced fairly quickly and that is why an exponential decay in TPAH is observed. These results also point out the need for high-energy dispersions to be monitored carefully during the course of a study where recurrent renewals may be needed at very short time intervals to maintain dosing consistency. 


\section{TPAHs-WAFs}
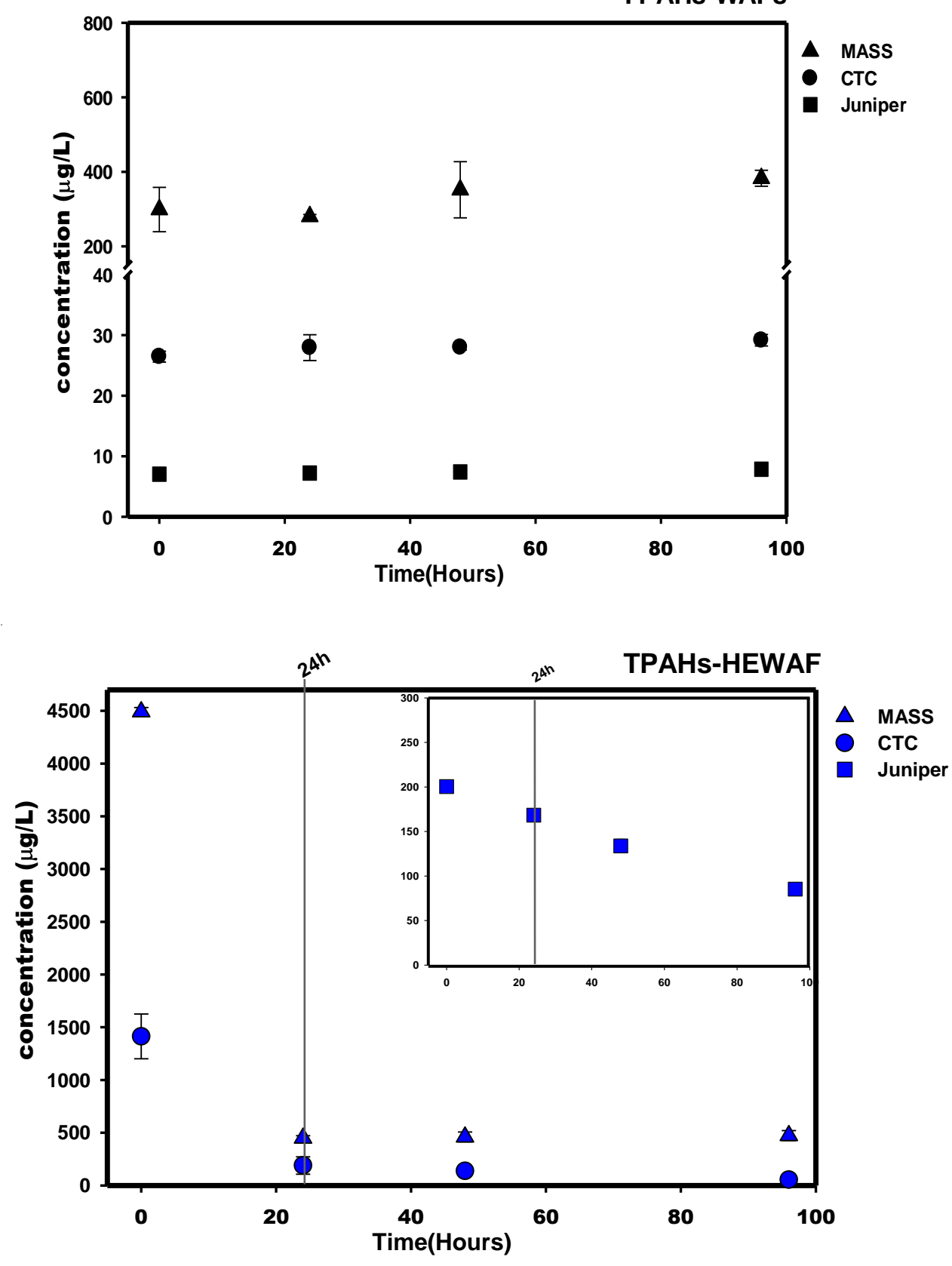

Figure 11: Stability of WAF preparations (top) and HEWAF preparations (bottom) measured as total polycyclic aromatic hydrocarbons (TPAH) concentrations over 96 hours for all oils, MASS = triangle, $\mathrm{CTC}=$ circle and Juniper= square. The inset shows an expanded view for the Juniper oil due to the overall lower concentrations present in the high energy preparation. 
Table 1: Percentage of TPAH loss at T24 compared to T0 (\% TPAH losses) for all oils and treatments.

\begin{tabular}{cccc}
\hline Treatments & MASS & Oils & CTC \\
\hline WAF & 6.26 & 5.63 & JUNIPER \\
CEWAF & 26.0 & 32.4 & 17.0 \\
HEWAF & 90.0 & 86.6 & 16.0 \\
SHEWAF & 90.4 & 89.9 & 55.8 \\
\hline
\end{tabular}

\subsubsection{Effects of filtration for the preparation of water soluble fractions (WSFs)}

Generally, toxicity testing of the dissolved fractions of oil have been based on WAF preparations following the CROSERF low energy stirring protocol (Singer et al., 2000) or WSFs generated by excluding oil droplets with the use of physical barriers (Nordtug et al., 2011a). The initial characterization of the low energy WAFs showed that low energy WAFs did produce droplet free solutions to the limit of optical detection. However, the generation of droplet free WSFs is mainly dependent on the efficiency of the filtration system. Complete physical characterization of WSFs used in toxicology laboratory tests is uncommon and it is often uncertain whether the exposure media still contain residuals of oil droplets. The WSFs were generated from the different preparations via tandem filtration using the combination of a filter supplemented with loose glass fibers to prevent much of the oil droplets from reaching the filters. The presence of droplets in the WSFs was examined optically by Epi-Fluorescence microscope images and fluorescence EEMs. Both techniques revealed that droplets were successfully removed from all treatments; examples for MASS oil are shown in appendix 2. 
In addition, TPAH concentrations of the WSFs for the different preparations were compared to those of the WAFs prepared from each test material. The results showed that WSFs were within $(100 \pm 30) \%$ relative to WAFs for the majority of the treatments with the exception of CEWAFs for both weathered oils (Fig.12). The significance of the $30 \%$ is related to the acceptance criterion for the analysis of sample replicates.

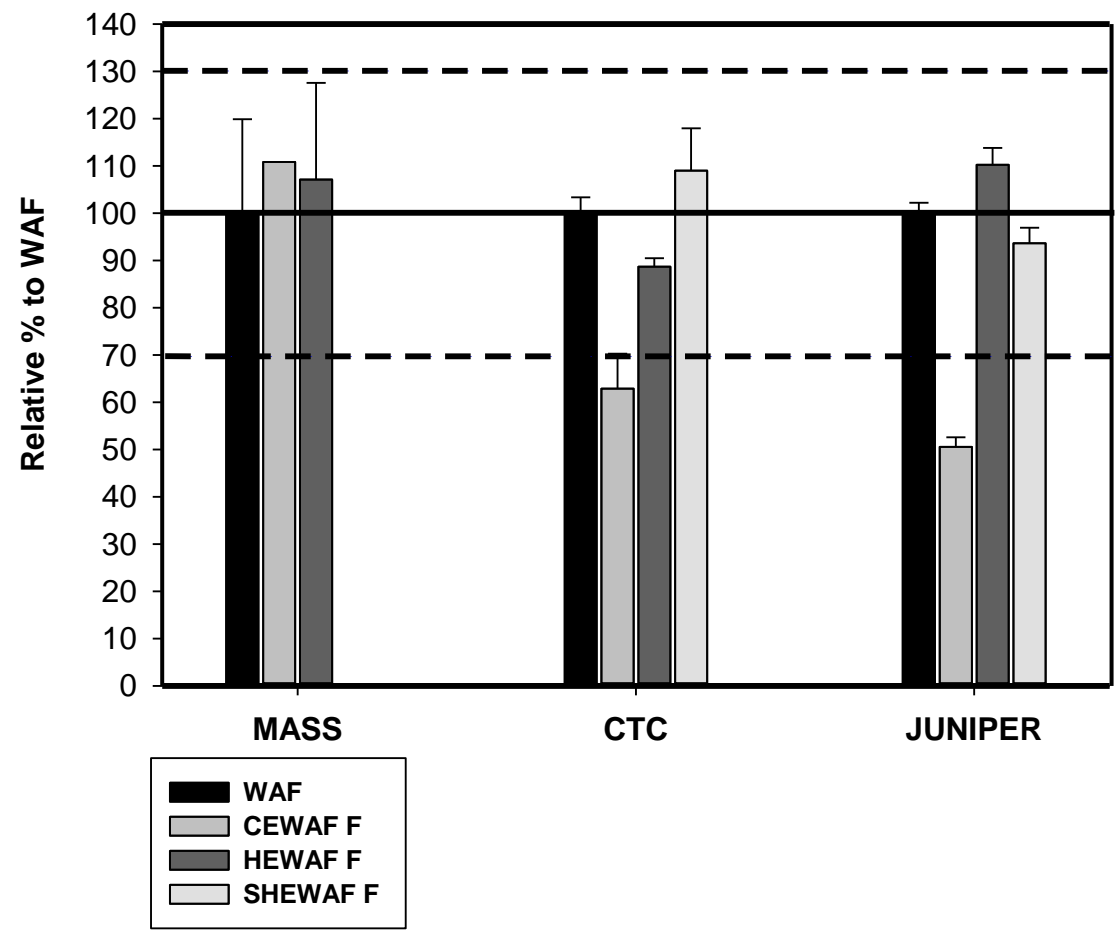

Figure 12: Comparison of WSF to WAF for all treatments and oils. Solid black line indicates $100 \%$ of WAF and dash black lines are $\pm 30 \%$ boundaries.

The TPAH and $\sum$ Tricyclic PAH concentrations for all the 1:1000 WAF and HEWAF-WSF preparations reported here were consistent with each other (Table 2). The PAH concentrations for HEWAF-WSF were compared to values reported by Brette et al. (2014) using the same oils and preparation methods and it was found that TPAH values 
of MASS oil were comparable $(320 \pm 61.0 \mu \mathrm{g} / \mathrm{L}$ vs. $303.3 \pm 22.8 \mu \mathrm{g} / \mathrm{L})$, but those from weathered oils differ significantly. Reported values for TPAHs and $\sum$ Tricyclic PAHs in slick A (CTC) and Slick B (Juniper) were 3 times and 16 times higher than the concentrations measured in this study, respectively. Chrysenes have a low solubility (log Kow > 5.8) in water but are prevalent in the weathered oil and their detection in the weathered oil WAFs described in Brette et al. (2014) is an indication of the presence of residual oil droplets in the exposure media. High-energy preparations with the weathered oils do generate droplets with average sizes in the range of 1-2 $\mu \mathrm{m}$ and some of them could be smaller than the filter used to retain the oil droplets leading to the potential presence of particulate oil in the WSFs. Both the presence of chrysene and elevated amounts of $\sum$ Tricyclic PAHs with respect to WAFs are evidence of the presence of oil droplets in the test solutions. Faksness et al. (2015) showed that for reported TPAH concentrations in WAFs for the same set of oils, the concentrations of the dissolved fractions decreases as a function of increased weathering and are more consistent with the results for the WSFs produced by the enhanced filtration process shown in this study.

Table 2: Average of TPAH concentrations and $\sum$ tricyclic PAH concentrations in WAFs and HEWAF-WSF for all oils.

\begin{tabular}{|c|c|c|c|c|}
\hline \multicolumn{3}{|c|}{ WAF } & \multicolumn{2}{|c|}{ HEWAF-WSF } \\
\hline Oil & $\begin{array}{l}{ }^{\mathrm{a}} \mathrm{TPAHs} \\
(\mu \mathrm{g} / \mathrm{L})\end{array}$ & $\begin{array}{c}{ }^{\mathrm{b}} \sum \text { Tricyclic PAHs } \\
(\mu \mathrm{g} / \mathrm{L})\end{array}$ & $\begin{array}{l}{ }^{\mathrm{a}} \mathrm{TPAHs} \\
(\mu \mathrm{g} / \mathrm{L})\end{array}$ & $\begin{array}{l}{ }^{\mathrm{b}} \sum \text { Tricyclic } \\
\text { PAHs }(\mu \mathrm{g} / \mathrm{L})\end{array}$ \\
\hline MASS & $298.8 \pm 59.4$ & $15.6 \pm 2.64$ & $320 \pm 61.0$ & $14.4 \pm 0.49$ \\
\hline $\begin{array}{l}\text { CTC } \\
\text { JUNIPER }\end{array}$ & $\begin{array}{l}26.5 \pm 0.89 \\
7.02 \pm 0.16\end{array}$ & $\begin{array}{l}14.2 \pm 0.56 \\
5.11 \pm 0.14\end{array}$ & $\begin{array}{l}23.5 \pm 0.47 \\
7.74 \pm 0.25\end{array}$ & $\begin{array}{l}12.9 \pm 0.13 \\
5.32 \pm 0.05\end{array}$ \\
\hline
\end{tabular}




\subsubsection{Environmental relevance}

The TPAH concentrations for all preparations for all the oils were compared to the most extensive compilation (10,828 records) of chemical data publicly available (BP, 2014) from water samples that were collected in the Gulf of Mexico during the Deepwater Horizon accident in 2010 and analyzed for a number of standardized parameters (Fig. 13). Water column TPAH data are organized on a temporal basis and expressed in a logarithmic scale. The TPAH concentrations for all the preparations generated in this study are overlaid as colored circles representing the test materials (green=MASS, blue=CTC, pink=Juniper) and distributed in boxes representing the different preparations (WAF, CEWAF, HEWAF and SHEWAF).

The results show that the TPAH concentrations for all the preparations are above the vast majority of values reported in the database for the GOM water column during and after the Deepwater Horizon event with the exception of some samples collected during the early stages of incident response most likely containing large amounts of visible oil. The full strength preparations ranked above the $98^{\text {th }}$ centile of all samples in the database with the low energy WAF preparations made of the most weathered oil (Juniper) having the lowest TPAH concentration at $6.95 \pm 0.16 \mu \mathrm{g} / \mathrm{L}\left(98.6^{\text {th }}\right.$ centile $)$ and the MASS oil SHEWAF producing the highest TPAH concentrations at $3155 \pm 708 \mu \mathrm{g} / \mathrm{L}$ $\left(99.9^{\text {th }}\right.$ centile). High-energy preparations in particular, introduced increasingly high amounts of oil in particulate phase to the testing media compared to the traditional WAF and CEWAF preparations (CTC WAF=25.7 $\pm 0.9 \mu \mathrm{g} / \mathrm{L}$; CTC CEWAF=73 $\pm 37 \mu \mathrm{g} / \mathrm{L}$; CTC $\mathrm{HEWAF}=1387 \pm 207 \mu \mathrm{g} / \mathrm{L}$ and CTC SHEWAF $=1717 \pm 38 \mu \mathrm{g} / \mathrm{L})$. 


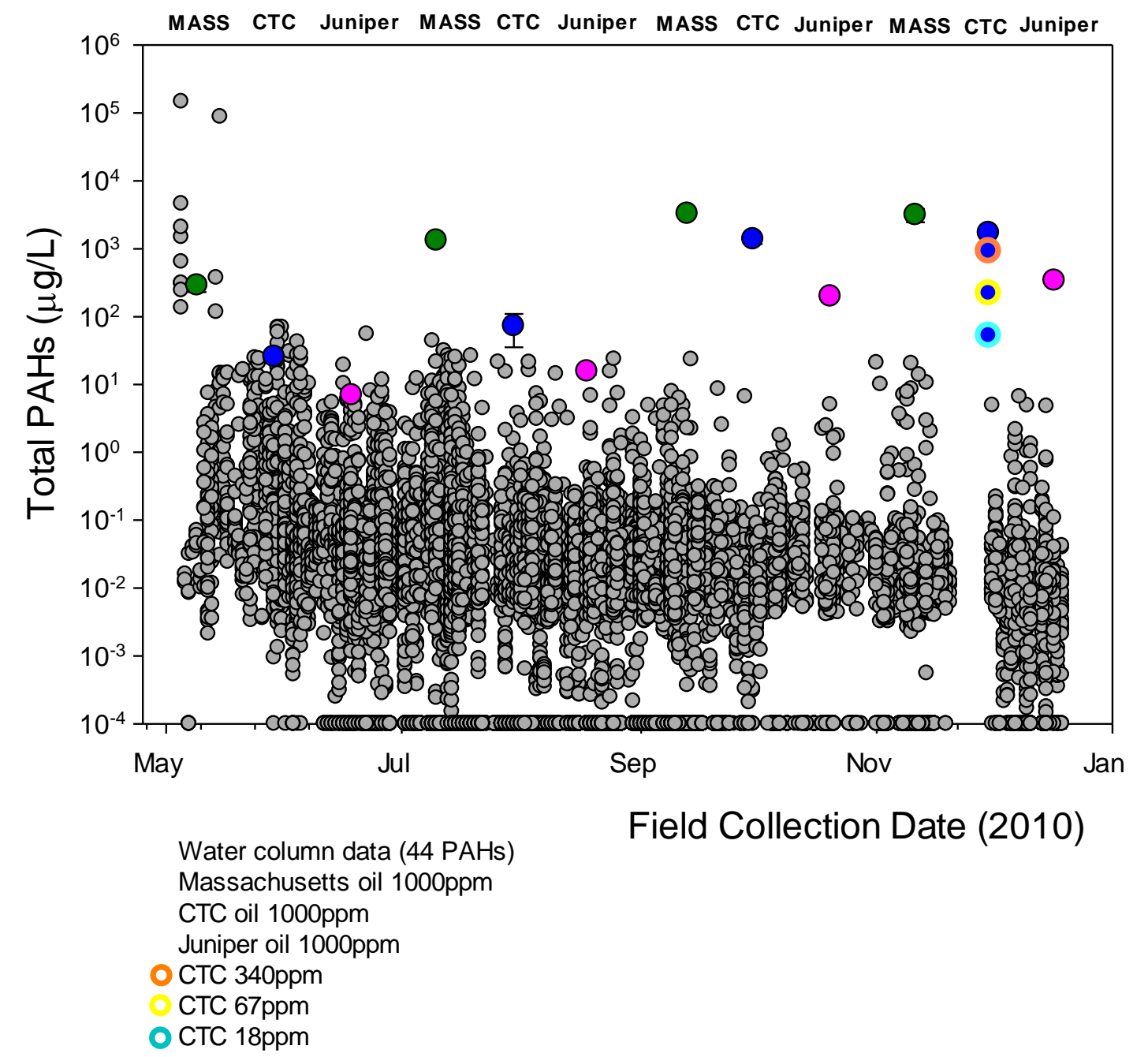

Figure 13: Total PAH concentrations in field samples collected from the Gulf of Mexico over time (May 2010 to December 2010) and TPAH concentrations for all treatments and oils in log scale. 
Figure 13 also includes a series of SHEWAF preparations using CTC oil at different loadings in where the lowest nominal loading prepared $(20 \mathrm{mg} / \mathrm{L})$ is the only high-energy preparation that produced TPAH concentrations similar to that of a CEWAF preparation. At these loadings, however, average droplet sizes in the HEWAF preparations are between 2 and 5 times smaller than those of the corresponding to the CEWAF preparations and probably not representative of any environmental conditions conducive to formation of droplets of weathered oil. The differences between environmental samples and all preparations can be clearly seen in Figure 14 where the scales have been transformed to linear and the PAHs are plotted as the sum of the 2-3 ring or 4-6 ring components. Water column data points are now presented as yellow and cyan symbols, respectively. The graphs (Fig.14) do show the dominance of low molecular weight PAHs (2-3 ring) in MASS oil preparations while dispersions prepared with CTC oil using high energy do introduce larger amounts of 4-6 ring PAHs. Higher concentrations of the 4-6 ring PAHs (dominated by chrysenes) are indicative of insoluble material present in the test solutions. As shown in Figure 14 (right) SHE/HEWAF preparations are 100-200 times more concentrated for MASS and CTC oils as compared to the CEWAF preparations. In addition, the same pattern is observed for the $\sum 2-3 \mathrm{PAH}$ rings which show that high energy methods introduce much higher TPAH concentrations in the test solutions with respect to the traditional WAF and CEWAF preparations (Fig.14, left) and the patterns are clearly a reflection of increased presence of insoluble components in the test material.

Several published studies have utilized the HEWAF methods and applied large dilution factors in order to reach potential environmentally realistic exposure to PAH 
concentrations similar to water samples collected from the GOM (Brette et al., 2014; Mager et al., 2014). Impaired cardiac responses on embryo-larval fish stages were found at $0.2 \%$ CTC HEWAF dilutions $(1.2 \mu \mathrm{g} / \mathrm{L}$ TPAH $)$ and swimming performance changes on juvenile Mahi-Mahi were reported for $2 \%$ CTC HEWAF dilutions (30 $\mu \mathrm{g} / \mathrm{L}$ TPAH) (Mager et al., 2014). In addition, specific dose-dependent effects in cardiac function were reported for pelagic fish at exposures to CTC HEWAFs prepared at 1 or $2 \mathrm{mg} / \mathrm{L}$ oil loading (3.4-13.8 $\mu \mathrm{g} / \mathrm{L}$ PAH) (Incardona et al., 2014). These concentrations, including the reported dilutions represent less than 450 of the 10,828 samples analyzed from the GOM during the DWH response phase as reported in the largest publicly available database. It will require dilutions of more than 1000 times for a Juniper low energy WAF preparation and up to 48,000 times for a MASS low energy WAF preparation to reach the $50^{\text {th }}$ centile of the TPAH concentrations reported in the database.

Furthermore, unless a detailed compound-specific analysis of the available chemistry data is conducted to separate the roles of dissolved or particulate components of the less soluble 3- and 4-ring PAHs, attributing specific effects to polycyclic aromatic hydrocarbons as TPAHs or single components is extremely difficult. In addition, as shown before special considerations have to be taken into account for the full characterization of high-energy preparations as minor changes in preparation protocols will greatly affect droplet distribution, stability and chemical composition of the exposure media over time. And high energy preparations may not be reflective of any real environmental conditions, predictive of effects of individual components and/or families of components or reflective of a single mode of action. 

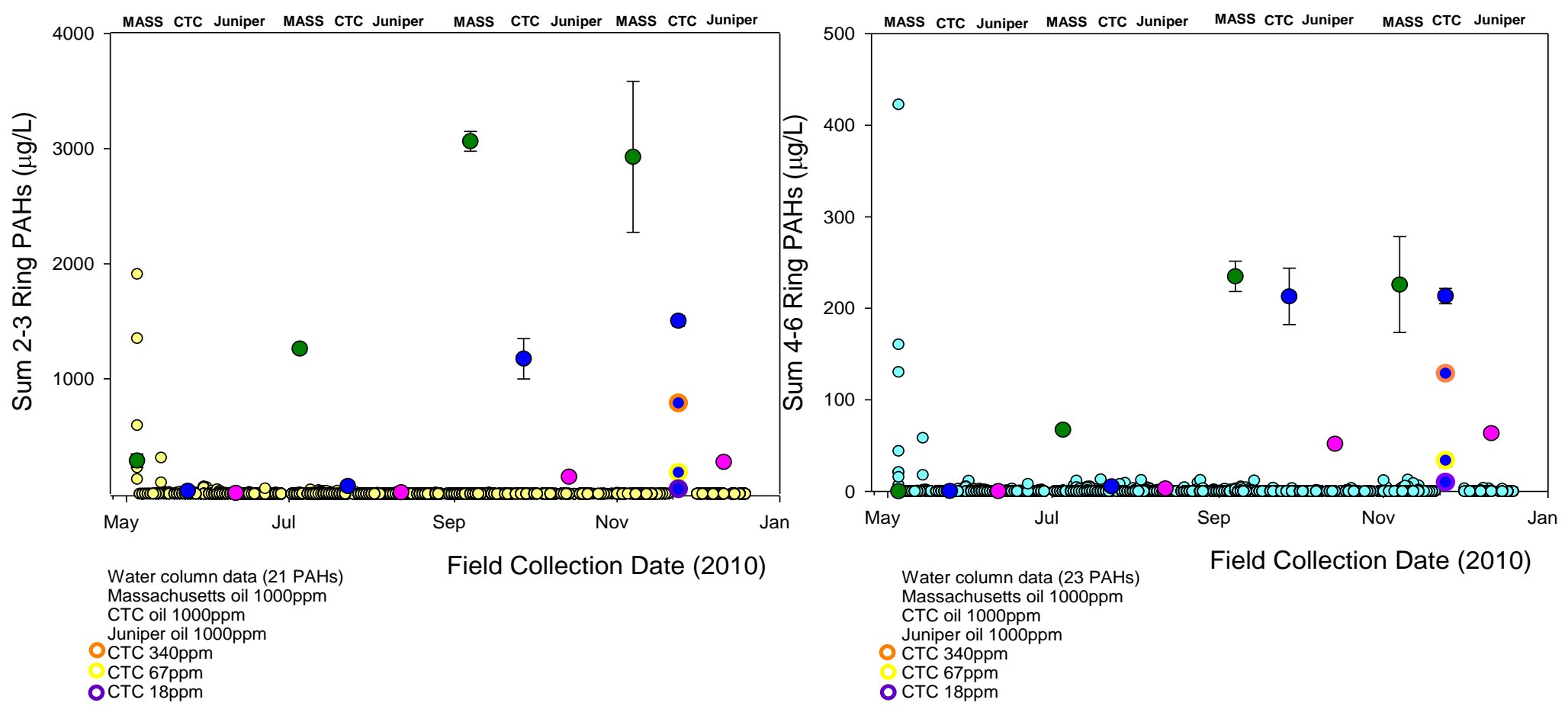

Figure 14: Sum of the concentration of 2-3 ring PAHs (left) and sum of the concentration of 4-6 ring PAHs (right) for field collected samples from the Gulf of Mexico and all the treatments prepared with all the oils in linear scale. 


\subsection{Conclusion}

In this study, a detailed systematic physical and chemical characterization was conducted for oil-water dispersions intended to be used as exposure media based on reported methodology (Aurand and Coelho, 2005; Brette et al., 2014; Incardona et al., 2014; Mager et al., 2014; Nordtug et al., 2011a; Singer et al., 2000) using discrete energy levels and multiple Macondo-related oils at different weathering stages. These results indicated that the traditional methods for preparation of exposure media (WAF and CEWAF) were found to be more stable and representative of both dissolved components and oil-water mixtures containing droplets, respectively. While, the new protocols involving high mixing energy (HEWAF and SHEWAF) required additional standardization and characterization steps as it was found that weathering stage and initial oil loadings do control droplet size formation and chemical composition of the test solutions. Because a well described protocol for SHEWAF/HEWAF preparations is still not available beyond the physical and chemical characterization provided in this work, it is of clear importance that toxicity test which utilized these particular protocols provide a full characterization. Because a relationship between oil loading and droplet size was found in the characterization of high-energy preparations and since the specific contribution of oil droplets to toxicity is still not well understood, these findings point out the importance of conducting a comprehensive physical and chemical characterization of exposure media in support of toxicology test for results among studies to be fully comparable.

This work also showed that water soluble fractions from the majority of the preparations were physically and chemically similar to their corresponding WAFs with 
respect to their bulk chemical characterization and to the limit of the optical detection. However, it is important to take into consideration when generating WSFs by filtration that additional physical surfaces (like loosely pack glass wool) should be used to retain the bulk of the insoluble material and filters with pores small enough to retain the small droplets should be used to prevent potential breakthrough of the smaller range of the insoluble particles often detected in the chemical analyses (Brette et al., 2014; Redman et al., 2012a). Until a better characterization of the available DWH-GOM water column individual-component chemistry data is produced the question of environmental relevance and the pursuit of studies under more realistic conditions continues to be a significant challenge and should be further explored. 


\section{A novel delivery system for studying the dissolution of individual and combined polycyclic aromatic hydrocarbons (PAHs) from micron-size droplets}

\subsection{Introduction}

Polycyclic aromatic hydrocarbons (PAHs) are a group of compounds consisting of two or more fused aromatic rings which make up a significant portion of the aromatic compounds that are naturally found in petroleum sources (Kopecka-Pilarczyk and Correia, 2009). These PAHs are an important class of pollutants because of their ubiquity, toxic, carcinogenic and mutagenic properties (Manzetti, 2012; Nisbet and Lagoy, 1992). One way in which these petrogenic PAHs may enter the marine environment is either from accidental oil spills or through natural oil seepage. The characterization and quantification of these pollutants are of great interest because of their persistence in the aquatic environment and toxicity to aquatic organisms (Cachada et al., 2014).

Due to the low solubility of PAHs, most of them would be found in aquatic environments in insoluble phases or in the form of solid particulates (Levinson et al., 2005). For example, following an oil spill micron-size oil droplets may form naturally through the action of breaking waves. These droplets, which contain a mixture of different classes of hydrocarbons, may persist in the water column and increase the surface-to-volume ratio of the oil and the rate of compounds partitioning from the droplets into the water phase within their solubility limits (Ramachandran et al., 2004b). Despite this fact, most of the aquatic studies focus mainly on the occurrence of contaminants in the dissolved phase and there is a clear shortage of data on the 
distribution of these contaminants in the particulate phase of the water column (Rabodonirina et al., 2015).

Even though it has been found that oil droplets are not the main contributors to toxicity as much as the dissolved fraction, they may still exert toxicity to aquatic organisms by a different toxic mode of action (Nordtug et al., 2015). For example, ingestion of small oil droplets can be an additional route for uptake of oil components (Viaene et al., 2014). Adsorption of droplets to membranes can interfere with functional and physiological processes and contact with lipid surfaces can lead to an increase in the rate of transfer of toxic oil components to aquatic organisms (Brown et al., 2001; Gauthier et al., 2014). While, most of the oil toxicity has been attributed to the dissolved phase organic contaminants such as the low molecular weight and more water soluble PAHs due to their enhanced bioavailability (Sorhus et al., 2015), it has been hypothesized that oil droplets may act as a reservoir of dissolved hydrocarbons which is constantly replenishing the dissolved phase hydrocarbons into the aqueous media (Carls et al., 2008). Thus, oil droplets could not only increase the bioavailability but also influence the bioaccessibility of PAHs contained within the droplets.

Because of the natural complexity of crude oil, limited knowledge exists on the behavior of individual compounds present in oil droplets in the water column in terms of their dissolution rates and toxicity. Determining the dissolution behavior of these relatively insoluble toxicants is based solely on the equilibrium of the organic compounds between the oil droplet and aqueous phase or the oil droplet and a membrane (TirutaBarna et al., 2006). When exposed to both phases, the compounds will partition between the phases based on their affinity for each. The affinity for each phase will depend on the 
solubility of the compound and from the octanol -water partitioning coefficient (Kow), as compounds with high solubility will have a higher affinity for the aqueous phase than compounds that are more hydrophobic. However, the solubility behavior of a compound in a mixture may not be equivalent to the predicted solubility of the pure compound (Sterling et al., 2003). Thus, the partitioning of compound $i$ from a mixture can be described by Raoult's law, which states that the maximum aqueous phase concentration $\left(\mathrm{C}_{\mathrm{i}}\right)$ of a compound in a mixture is the product of the mole fraction of that compound in the mixture $\left(\mathrm{x}_{\mathrm{i}}\right)$ and the solubility of the pure compound $\left(\mathrm{S}_{i}\right)$ assuming an ideal mixture (Poulsen et al., 1992)

$$
\mathrm{C}_{\mathrm{i}}=\mathrm{X}_{i} \mathrm{~S}_{i}
$$

Understanding the dissolution of PAHs is critical for assessing exposure or contaminant toxicity to aquatic organisms. It is of great importance to measure the effects of not only single PAHs but different PAH mixtures as it has been suggested that PAH mixtures could show systematic effects (Engraff et al., 2011). Smith et al. (2013) also demonstrated that the individual toxicity of selected PAHs (anthracene, chrysene and benzo(a)pyrene) resulted in limited or no response, while binary and tertiary mixtures resulted in significant acute toxicity to Daphnia magna.

The proper testing of contaminants is important for proper risk assessment, therefore new dosing systems for aquatic toxicity is a necessity. Thus, in this study we describe a novel dosing mechanism to study the chemical behavior of single or mixed PAHs of concern directly from binary systems (droplet dispersions). This paper first describes and characterizes the generation of oil water dispersions (OWDs) using a synthetic oil prepared exclusively from hydrocarbons and different amounts of individual 
and mixed PAHs for which the complete composition of the material is known. Secondly, the use of micron-size droplets as the delivery system was assessed for rapid dissolution and establishment of aqueous phase equilibrium for several individual PAHs. Lastly, the application of the system was tested with a PAH mixture that resembled an actual crude oil.

\subsection{Experimental}

\subsubsection{Materials and reagents}

$\mathrm{N}$-alkanes $\left(\mathrm{C}_{7}-\mathrm{C}_{25}\right)$ and Pristane were obtained from different suppliers at $96+\%$ purity. The following PAHs were used: naphthalene (99\%, Aldrich), 2methylnaphthalene (95-97\%, Acros), 2,6-dimethylnaphthalene (99\%, Ultra Scientific), fluorene (98\%, Acros), phenanthrene (99.5\%, Aldrich), dibenzothiophene (98\%, Aldrich), 4-methyldibenzothiophene (96\%, Aldrich), 4,6-dimethyldibenzothiophene (95\%, Acros), pyrene (99\%, Sigma) and chrysene (98.3\%, Fluka). Unweathered Mississippi Canyon 252 (MC252) crude oil collected on August 15 ${ }^{\text {th }}, 2010$ from the Oil Barge Massachusetts during the Deepwater Horizon (DWH) incident was provided by BP (BP Exploration \& Production Inc.). Artificial filtered saltwater (pore size: $0.45 \mu \mathrm{m}$, salinity $=33 \mathrm{psu}$ ) was prepared with Instant Ocean ${ }^{\circledR}$ (Aquarium Systems, Mentor, OH) and used to generate the dispersions.

\subsubsection{Preparation of mixtures}

A multicomponent mixture consisting of pure alkanes $\left(\mathrm{C}_{7}-\mathrm{C}_{25}\right)$ and an isoprenoid (Pristane) was prepared by mixing equimolar volumes of the alkanes normalized to $\mathrm{C}_{7}$. The heavier molecular weight alkanes $\left(\mathrm{C}_{18}-\mathrm{C}_{25}\right)$ that were either in a wax or crystallized form were added directly to the mixture and allowed to dissolve by vigorous mixing. This 
mixture served as the surrogate or synthetic Alkane Oil (AO) matrix. Single PAHs (fluorene, pyrene and chrysene) were mixed with the $\mathrm{AO}$ at different concentrations. In addition, an $\mathrm{AO}$ solution containing $10 \mathrm{PAHs}$ was prepared at concentrations similar to the ones measured for the Macondo crude oil. The concentrations of the PAHs in the Macondo oil were obtained from the public database at www.gulfofdatabase.com and are included in appendix 3 for further reference.

\subsubsection{Analysis of mixtures by GC-FID and GC-MS}

The distribution of the alkanes in the AO was characterized with a Thermo Trace 1310 gas chromatography-flame ionization detector (GC-FID) fitted with an $\mathrm{Rxi}^{\circledR}-5 \mathrm{Sil}$ fused silica capillary column ( $30 \mathrm{~m} \times 0.25 \mathrm{~mm} \times 0.25 \mu \mathrm{m})$. A sample volume of $2 \mu 1$ was injected in splitless mode into the instrument. The inlet temperature was held at $325^{\circ} \mathrm{C}$, while the initial oven temperature was held at $40^{\circ} \mathrm{C}$, then raised at a rate of $7.5^{\circ} \mathrm{C} / \mathrm{min}$ to $215^{\circ} \mathrm{C}$ followed by a second raise at a rate of $10^{\circ} \mathrm{C} / \mathrm{min}$ to $320^{\circ} \mathrm{C}$ and held constant for 13 minutes. The carrier gas was set at a constant flow rate of $2.4 \mathrm{~mL} / \mathrm{min}$. The characterization of the $\mathrm{AO}$ mixtures prepared with the single and mixed PAHs was performed by gas chromatography-mass spectrometry (GC-MS) following the analytical method previously described in section 2.2.10.

\subsubsection{Preparation of dispersions}

Droplet dispersions of known concentrations and droplet size distributions (DSDs) were generated using an oil droplet generator system previously described by Nordtug et al. (2011a). Briefly, the AO gets pumped through the inlet of a series of chambers with connecting nozzles into a flow of filtered saltwater which moves through the chambers creating turbulent mixing. The saltwater flow rate was adjusted to $160 \mathrm{ml} / \mathrm{min}$ in order to 
produce droplets with sizes of $10 \mu \mathrm{m}$ in diameter. The AO loading was set to $22 \mu \mathrm{l} / \mathrm{min}$ in order to produce a theoretical oil concentration of approximately $100 \mathrm{mg} / \mathrm{L}$ based on an $\mathrm{AO}$ density of $0.74 \mathrm{~g} / \mathrm{mL}$. The $\mathrm{AO}$ was formulated to represent a light oil with an average density of $0.7-0.8 \mathrm{~g} / \mathrm{mL}$. Oil water dispersions (OWDs) and water soluble fractions (WSFs) were generated with the different mixtures containing the individual PAHs in $\mathrm{AO}$, the PAH mixture in $\mathrm{AO}$ and the Macondo crude oil. For the dispersions generated with the Macondo crude oil, the oil loading was adjusted to $18 \mu \mathrm{l} / \mathrm{min}$ in order to account for difference in the oil density $(0.83 \mathrm{~g} / \mathrm{mL})$. WSFs were created by filtering the droplet dispersions under mild vacuum using a tandem system of filters GFD/GFF (pore size of $3.5 \mu \mathrm{m}$ followed by pore size $0.7 \mu \mathrm{m}$ ) and a large amount of loosely packed glass fiber to retain the droplets before they reach the filter surface to prevent droplet breakthrough.

\subsubsection{Physical characterization of dispersions}

Samples were collected immediately after preparation and characterized for DSD, oil concentration, and the qualitative assessment of both dissolved fractions and particulate phase using the characterization methods detailed in sections 2.2.6, 2.2.7, and 2.2.8. Briefly, droplet concentrations and size distributions were measured using a Coulter Counter Multisizer 4 (Beckman-Coulter Inc., Miami, FL) equipped with an 100 $\mu \mathrm{m}$ size aperture which allowed particle sizing within the range of 2-60 $\mu \mathrm{m}$. An IX71 epi-fluorescence inverted microscope (Olympus, Center Valley, PA) was utilized to

characterize the individual droplets in the dispersion by capturing both bright field and fluorescence images. A Fluoromax-4 scanning spectrofluorometer (Horiba Jobin Yvon 
Inc.) was used to acquire excitation-emission matrix (EEM) spectra in order to monitor the dissolved versus droplet phase of the dispersions.

\subsubsection{Chemical characterization of dispersions}

In order to quantify the concentrations of PAHs, samples were collected (approximately $300 \mathrm{~mL}$ each) and reserve for liquid-liquid extraction and analyzed by GC-MS as previously described in sections 2.2.9 and 2.2.10. WSFs prepared with single PAHs (fluorene, pyrene and chrysene) were measured by utilizing an online SPE-LCAPPI-MS/MS method described by Ramirez et al. (2014) where samples did not require the extraction process and results were obtained in a faster time compared to the GC-MS method.

\subsection{Results and Discussion}

\subsubsection{Characterization of the Alkane Oil (AO)}

The GC chromatogram shown in Figure 15 shows the distribution of the alkanes in the AO. The labeled peaks correspond to the added internal standards which are as followed: Dodecane_d26, Hexadecane_d34, Eicosane_d42, Androstane and pterphenyl_d14. The n-alkanes $\left(\mathrm{C}_{7}-\mathrm{C}_{25}\right)$ were chosen as the backbone of the synthetic oil because of their negligible solubility ensuring that the alkanes within the droplets would not partition into the water phase but remain in the droplet phase. Such process mimics the mechanism of oil droplets that are formed with an actual oil, as those oil droplets mostly contained insoluble compounds. In addition, the $\mathrm{AO}$ is representative of the alkanes that are abundantly present in many crude oils or petroleum products. 


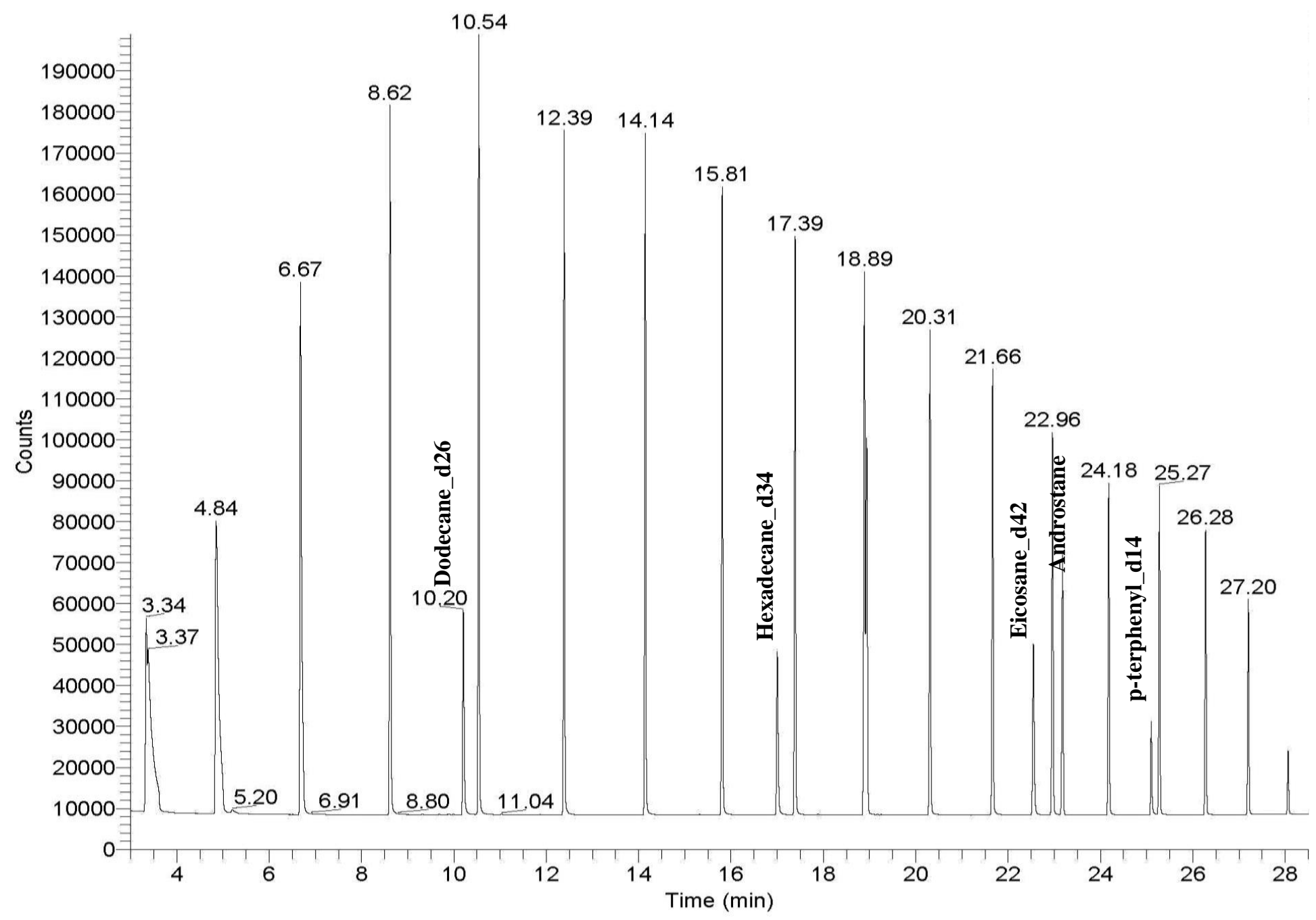

Figure 15: Chromatogram of the Alkane Oil (AO) obtained by GC-FID showing the distribution of the alkanes and labeled internal standards. 


\subsubsection{Droplet characterization of the oil water dispersions (OWDs) prepared with the Alkane Oil (AO) containing pyrene}

In order to test the dissolution and equilibration of individual polycyclic aromatic hydrocarbons from $\mathrm{AO}$ droplets into the aqueous media, it was important to first successfully generate OWDs with the AO containing both the right droplet size distributions and the correct amount of a selected PAH. Pyrene was selected as the first test substance due to its limited solubility and its persistence in marine and estuarine environments at low concentrations in the ppb-ppt range (Almeida et al., 2012). Solutions of pyrene mixed with $\mathrm{AO}$ were prepared at 5 nominal concentrations between 163 to $1855 \mathrm{mg} / \mathrm{L}$ (Table 3).

The oil droplet generator system was chosen because of its ability to prepare dispersions of consistent droplet sizes and reproducible concentrations using crude oils with densities between 0.83 to $0.9 \mathrm{~g} / \mathrm{mL}$ (Brakstad et al., 2015; Hansen et al., 2012; Nordtug et al., 2011a; Nordtug et al., 2011b). The system was set to generate dispersions of approximately $10 \mu \mathrm{m}$ median droplet sizes with theoretical oil concentration of 100 $\mathrm{mg}$ oil/L of saltwater for a series of OWDs containing increasing amounts of pyrene in the AO. Droplet characterization using a Coulter Counter particle analyzer was immediately performed for each of the OWDs in order to demonstrate the generation of droplets and to determine both the DSDs and oil concentration. The results are shown in Table 3, the DSD and oil concentrations were in good agreement between the theoretical and the measured values with averages of $9.17 \mu \mathrm{m}$ and $107 \mathrm{mg} / \mathrm{L}$, respectively. 
Table 3: Characterization of oil water dispersions containing pyrene

\begin{tabular}{|c|c|c|c|}
\hline $\begin{array}{l}\text { Concentration of } \\
\text { pyrene in } \mathrm{AO}(\mathrm{mg} / \mathrm{L})^{\mathrm{a}}\end{array}$ & $\begin{array}{l}\text { Measured concentration of } \\
\mathrm{AO} \text { in dispersion }(\mathrm{mg} / \mathrm{L})^{\mathrm{b}}\end{array}$ & $\begin{array}{l}\text { Nominal concentration of total } \\
\text { pyrene in dispersion }(\mu \mathrm{g} / \mathrm{L})^{c}\end{array}$ & $\begin{array}{l}\text { Droplet size distribution } \\
(\mu \mathrm{m})^{\mathrm{d}}\end{array}$ \\
\hline 163 & $100 \pm 6.1$ & $21.9 \pm 1.41$ & $9.03 \pm 0.17$ \\
\hline 489 & $105 \pm 1.2$ & $69.3 \pm 0.92$ & $8.89 \pm 0.07$ \\
\hline 827 & $110 \pm 4.7$ & $123 \pm 4.24$ & $9.06 \pm 0.04$ \\
\hline 1103 & $107 \pm 3.9$ & $160 \pm 6.36$ & $9.51 \pm 0.15$ \\
\hline 1855 & $114 \pm 14$ & $268 \pm 35.3$ & $9.35 \pm 0.14$ \\
\hline
\end{tabular}

${ }^{a}$ Nominal concentrations of pyrene in the AO. ${ }^{b, d}$ Measured concentration of AO in the dispersions and DSDs were obtained from Coulter Counter analysis. ${ }^{c}$ Nominal concentration of total pyrene in dispersions was calculated from the concentration of $\mathrm{AO}$ in the dispersion and the concentrations of pyrene in AO.

Evidence of the presence of droplets was obtained by optical and fluorescence microscopy. The UV epi-fluorescence illumination unit containing a band pass excitation filter of 330-385 nm was used to account for the presence of pyrene in the droplets as pyrene has a high quantum yield and fluoresces in the blue-violet region of the spectrum (335 nm) (Zhang et al., 2004). Figure 16 shows the microscope images of the dispersed droplets containing three different concentrations of pyrene in the AO in bright field (top) and UV fluorescence illumination (bottom). The bright field images clearly depict the presence of $\mathrm{AO}$ droplets in all the dispersions, while the fluorescent images confirm that increasing the concentration of pyrene in the AO leads to an increase in the fluorescence of the dispersed droplets. This behavior can be clearly explained by the physio-chemical properties of pyrene, a four-ring PAH, with a relatively high log Kow value of 4.95 that is sparingly soluble in seawater (Brown et al., 2001). Due to its limited solubility, only a small portion of pyrene will equilibrate with the aqueous phase leaving a large portion of the pyrene in the AO droplets. These results clearly confirm both the successful generation of dispersed droplets composed of the AO and the presence of pyrene in the droplets. 


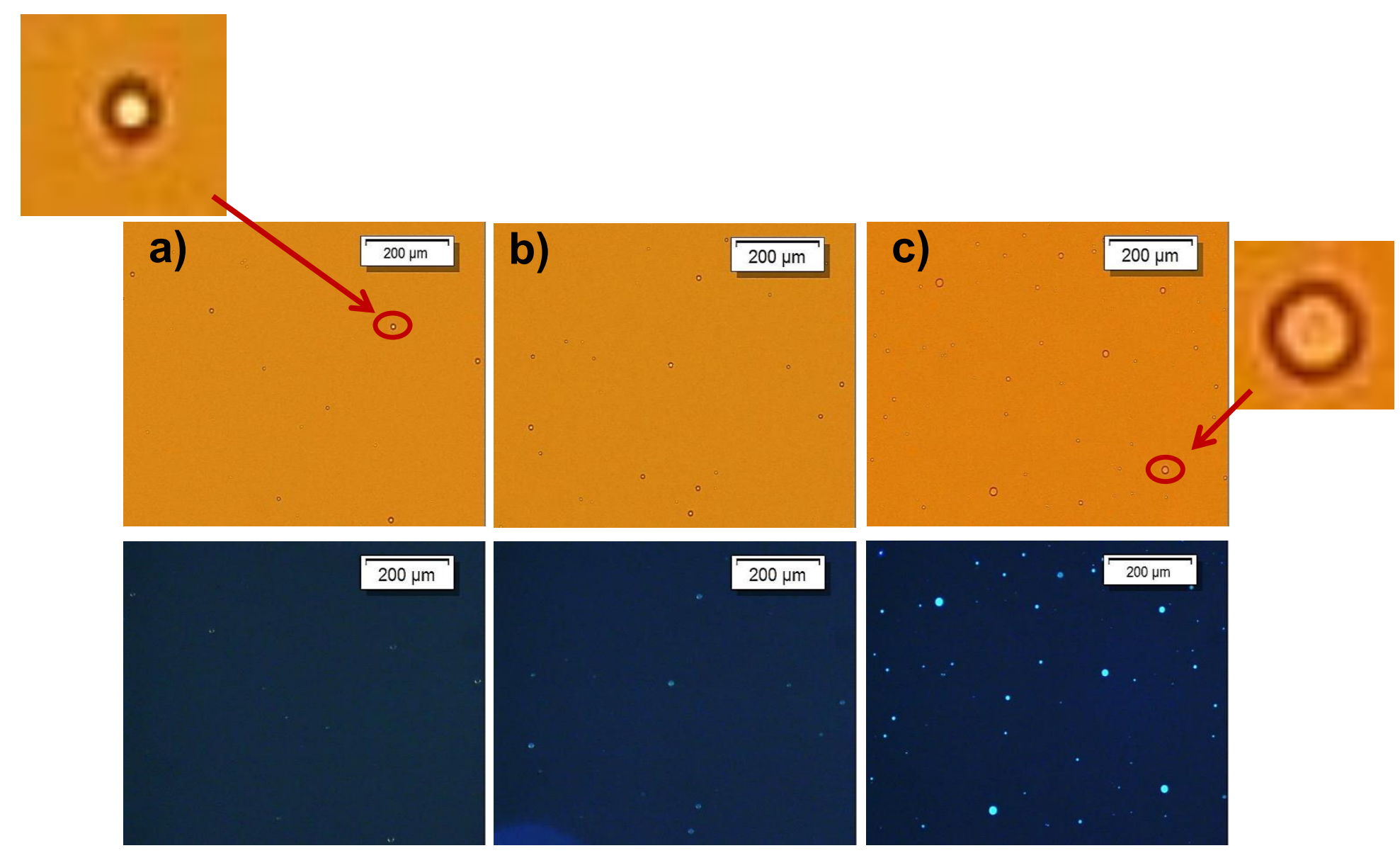

Figure 16: Microscope images of Alkane Oil (AO) droplets for three concentrations of pyrene in the AO at a) $163 \mathrm{ppm}$ b) $827 \mathrm{ppm}$ and c) $1855 \mathrm{ppm}$ in bright field (top) and UV fluorescence illumination (bottom). The inlet images are zoomed in of the droplets corresponding to the droplets that are circled in the bright field images. 


\subsubsection{Fluorescence characterization of water soluble fractions (WSFs) and oil water dispersions (OWDs) containing pyrene}

Due to the unique fluorescence spectral features of PAHs, EEM

fluorescence spectroscopy was used to monitor pyrene directly from the aqueous solutions without further sample manipulation. EEMs expressed as contour plots are shown in Figure 17 for one of the mid-range AO-pyrene mixtures ( $827 \mathrm{ppm}$ ) for both preparations containing droplets (OWD) and the solution consisting of only the dissolved fraction (WSF). Figure 17 (top) clearly depict the reduced fluorescence signature of pyrene in the WSF centered at an Ex/Em of 335/370 nm, while the OWD (Fig.17, bottom) exhibit strong fluorescence intensity signals spanning over a wider range of emission wavelengths from $400-530 \mathrm{~nm}$ which could be attributed to the presence of undissolved pyrene in the droplet phase. A similar emission spectrum for pyrene in aqueous solutions has been previously reported by Xie et al. (2007).

These findings suggest that the alkane oil matrix does not alter the fluorescent spectral characteristics of pyrene in aqueous solutions but acts in a similar fashion as a crude oil droplet. However, the fluorescence signatures will be highly dependent on the quantum yield of the individual PAHs. Thus, PAHs which have a low quantum yield may be hard to examine by fluorescence spectroscopy. In such case, the difference between the dissolved and droplet phase will be hard to distinguish at lower concentrations of the PAH in the Alkane Oil. Additionally, in the case of a mixture of PAHs in the AO the fluorescence characteristics of each individual PAH may not be able to be determined. 

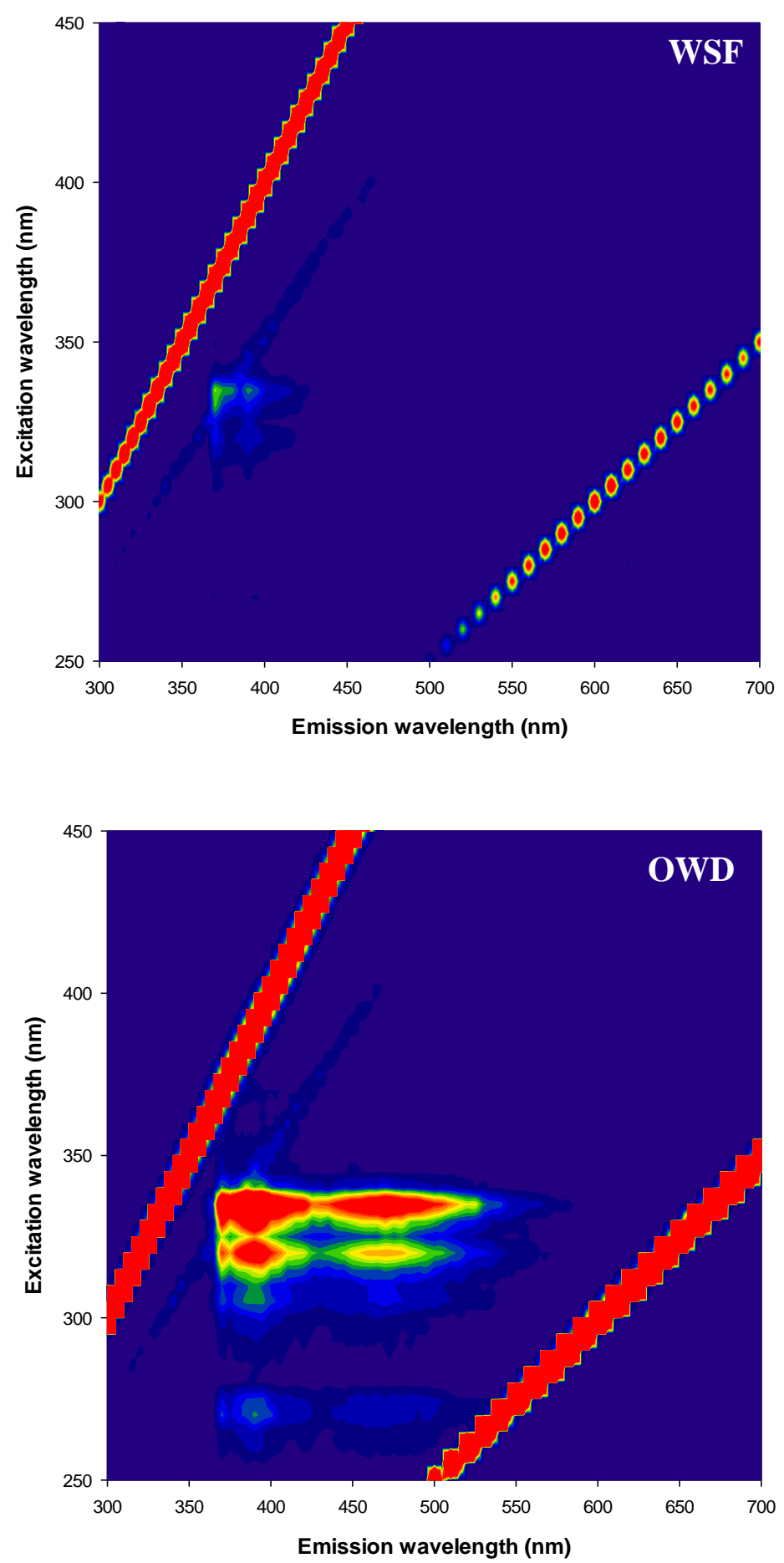

Figure 17: Contour plots for the filtered WSF (top) and unfiltered OWD (bottom) for dispersions prepared with AO-PYR $(827 \mathrm{ppm})$. The diagonal red lines are due to first and second order Rayleigh scattering produced by the instrument and not related to the data. 
Additionally, the fluorescence response of individual PAHs at their corresponding Ex/Em wavelengths maxima can be plotted against increasing concentrations of the PAH in order to estimate concentrations in the aqueous phase. This could be used to evaluate the potential loss of the PAH during toxicology testing or to determine the ratios between the dissolved and droplet phase in almost real time. For example, the fluorescence intensity of pyrene at Ex/Em $(335 / 370 \mathrm{~nm})$ was plotted against increasing concentration values of pyrene and a linear trend was observed for the WSF $\left(\mathrm{r}^{2}=0.99\right)$ as shown in Figure 18A. However, due to fluorescence quenching and self-absorption the trend deviated from linearity for the OWD (Fig. 18B) at concentrations higher than $827 \mathrm{mg} / \mathrm{L}$ of pyrene in the $\mathrm{AO}$ or an equivalent of $123 \mu \mathrm{g} / \mathrm{L}$ of total pyrene in the dispersion. This shows that fluorescence response could be used as an alternative to monitor PAHs based on their fluorescence characteristics in a well-defined system such as the one described in this study.

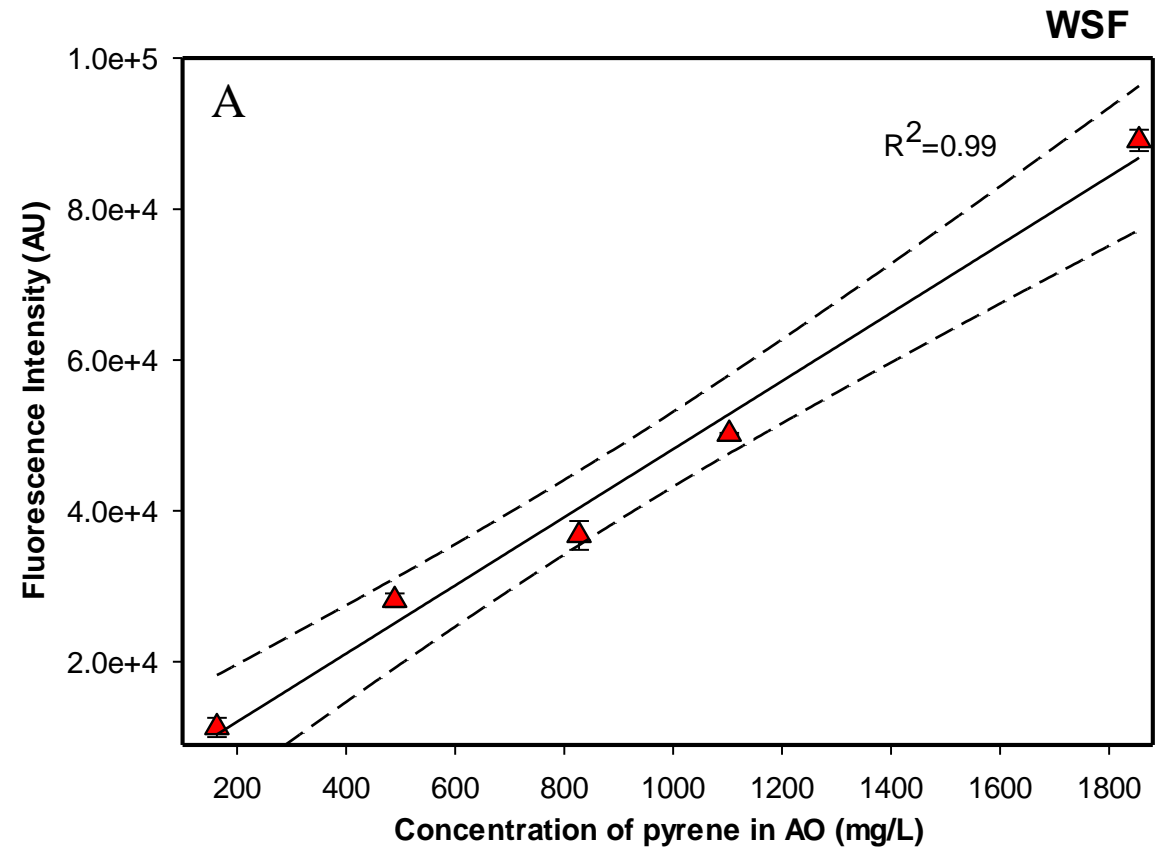




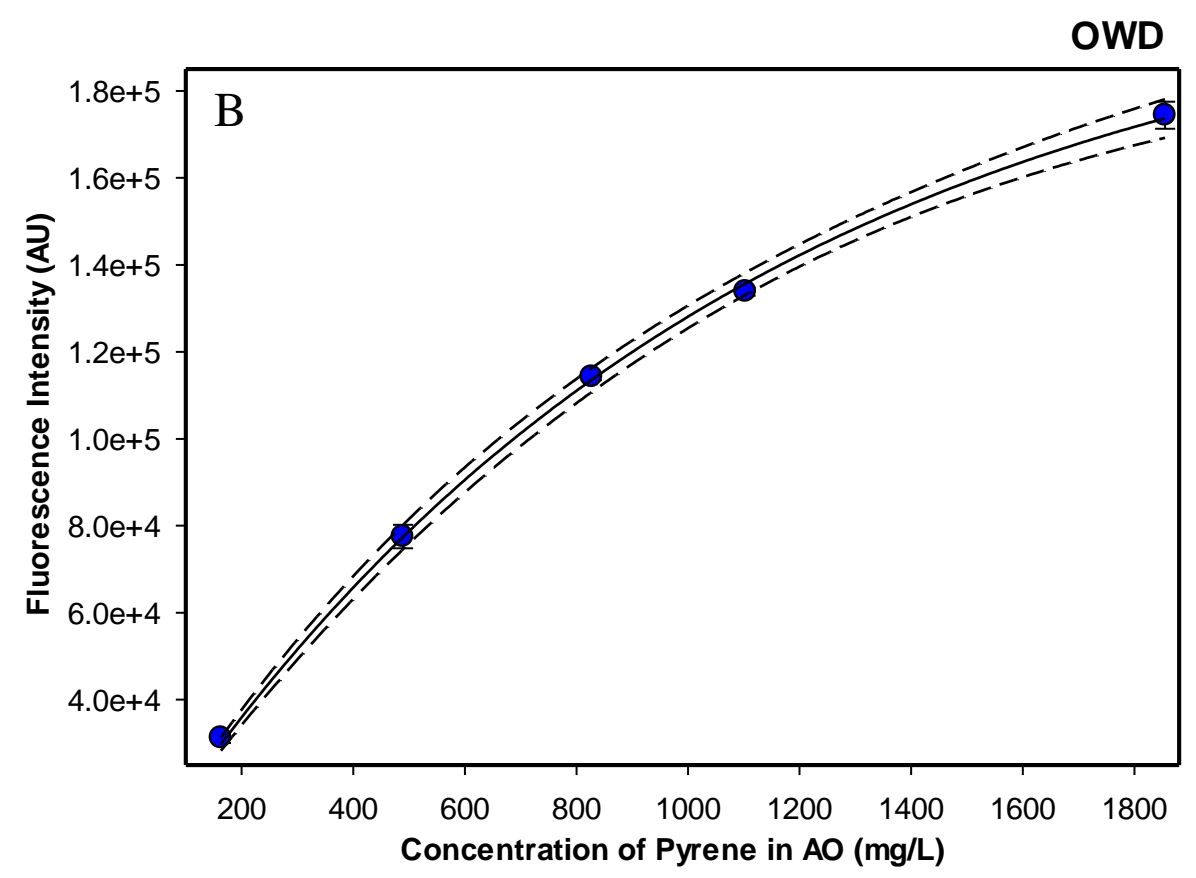

Figure 18: Relationship between the fluorescence intensity and concentration of pyrene in $\mathrm{AO}$ for the WSF (A) and OWD (B). Solid line is linear regression and dashed line is $\pm 95 \%$ confidence limits.

\subsubsection{Chemical characterization of oil water dispersions (OWDs) prepared with the Alkane Oil (AO) containing Pyrene}

Based on the measured concentrations of pyrene in the $\mathrm{AO}$ and the concentration of $\mathrm{AO}$ in the dispersions, the nominal concentrations of total pyrene in each of the dispersions were calculated and shown in Table 3. The measured concentrations of total pyrene, sum in both dissolved and droplet phase, for all the OWDs were obtained by processing the samples by liquid-liquid extraction and analyzing the extracts by GC/MS. The measured concentrations of total pyrene were compared to the nominal concentrations of total pyrene in the dispersions as shown in Figure 19. The results showed that the measured concentrations were fairly consistent with the nominal concentrations of total pyrene for the dispersions that contained lower amounts of pyrene 
in the AO (3\% RPD) and an increase in deviation was observed at higher concentrations of pyrene in the AO (up to $34 \%$ RPD). This may be explained by the potential removal of droplets from the system by attachment to the sample container walls during sample processing. The removal of droplets that contained higher pyrene content $(1855 \mathrm{mg} / \mathrm{L}$ of pyrene in AO) will impact the measured concentration much more than the removal of droplets containing lower amounts of pyrene $(163 \mathrm{mg} / \mathrm{L}$ of pyrene in $\mathrm{AO})$.

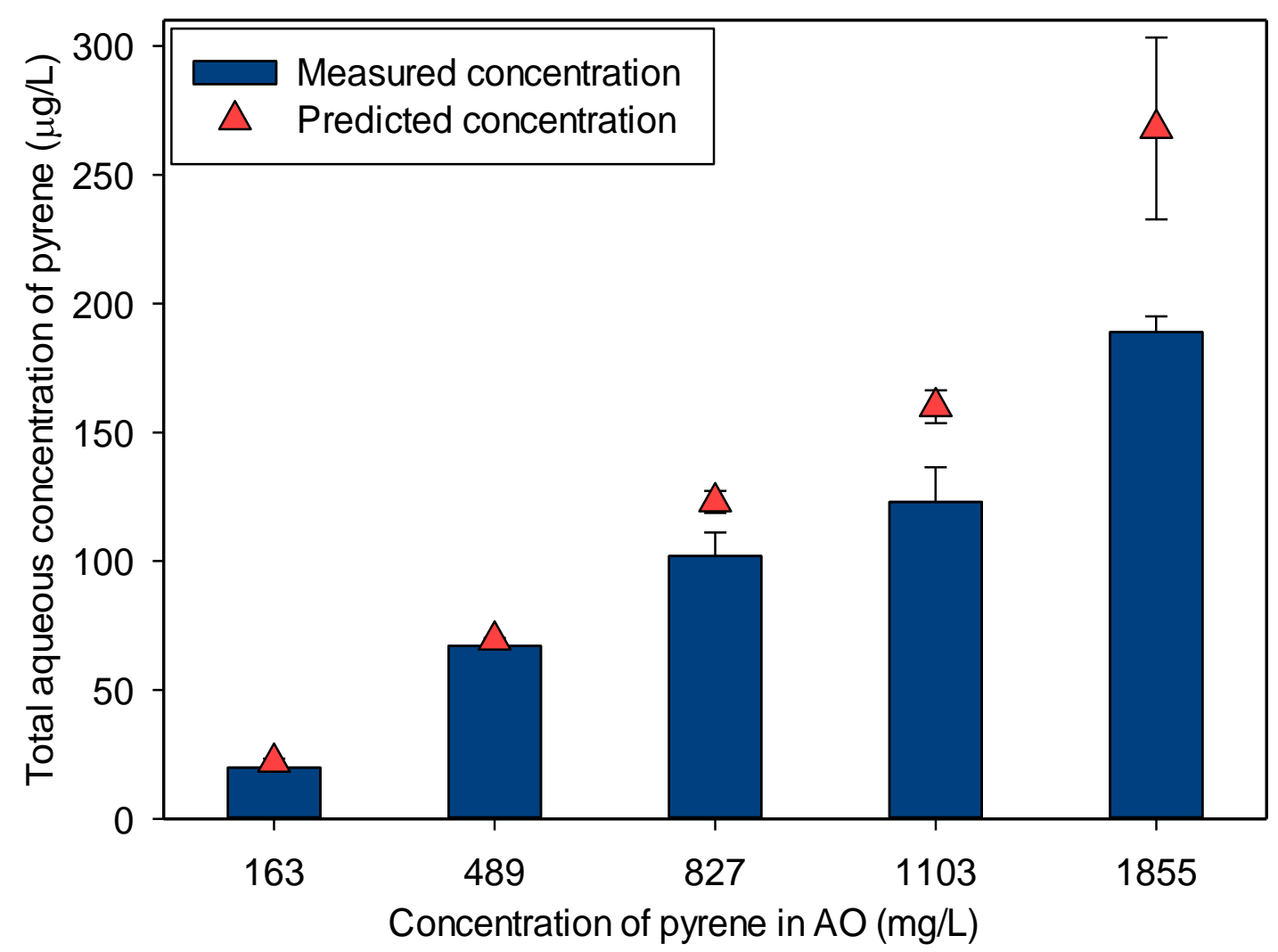

Figure 19: Comparison between the measured and predicted concentration of total pyrene (sum in both dissolved and droplet phase) for the dispersions prepared with different amounts of pyrene in the AO.

\subsubsection{Dissolution and equilibration of individual polycyclic aromatic hydrocarbons (PAHs) from the AO droplets into the water phase}

The dissolution of compounds from the $\mathrm{AO}$ droplets into the water phase is a complex process that involves a series of interactions between the hydrocarbon molecules 
that are non-polar and the surrounding water molecules which are polar (Lehr et al., 2010). Based on the common rule of "Like Dissolves Like", polar molecules will dissolve in water, while the non-polar molecules will remain in the Alkane Oil. Mid molecular weight PAHs are sparingly soluble and thus they can partition from the oil droplets into the water phase until equilibrium is reached between both phases. Furthermore, according to Raoult's law the dissolved aqueous concentration of compounds in a mixture depends on the aqueous solubility of each of the compound and their mole fraction. To confirm Raoult's law, the aqueous concentrations for fluorene, pyrene and chrysene were measured at increasing mole fractions in the $\mathrm{AO}$ as shown in Figure 20. As expected, a good linear relationship was observed as an increase in mole fraction in the $\mathrm{AO}$ resulted in a proportional increase in the aqueous concentrations.

It is important to point out that even though equilibrium between the droplet and dissolved phase was reached; the concentrations observed in the aqueous phase were all below the compounds predicted solubility. Furthermore, the effect of aqueous solubility on the dissolved aqueous concentrations of the PAHs was examined by calculating the ratios (expressed as percentages) of the dissolved aqueous concentrations versus the bulk concentrations of the PAHs in the dispersion. Fluorene which is fairly soluble in water dissolved between 13.1-16.7 \% of its predicted solubility with an average of $14.7 \%$, followed by pyrene (2.06-2.49\%, average of $2.23 \%)$ and lastly chrysene $(0.5-0.7 \%$, average of $0.6 \%$ ). These results are in agreement with Raoult's law, indicating ideal behavior for these compounds mixed individually with the AO. 

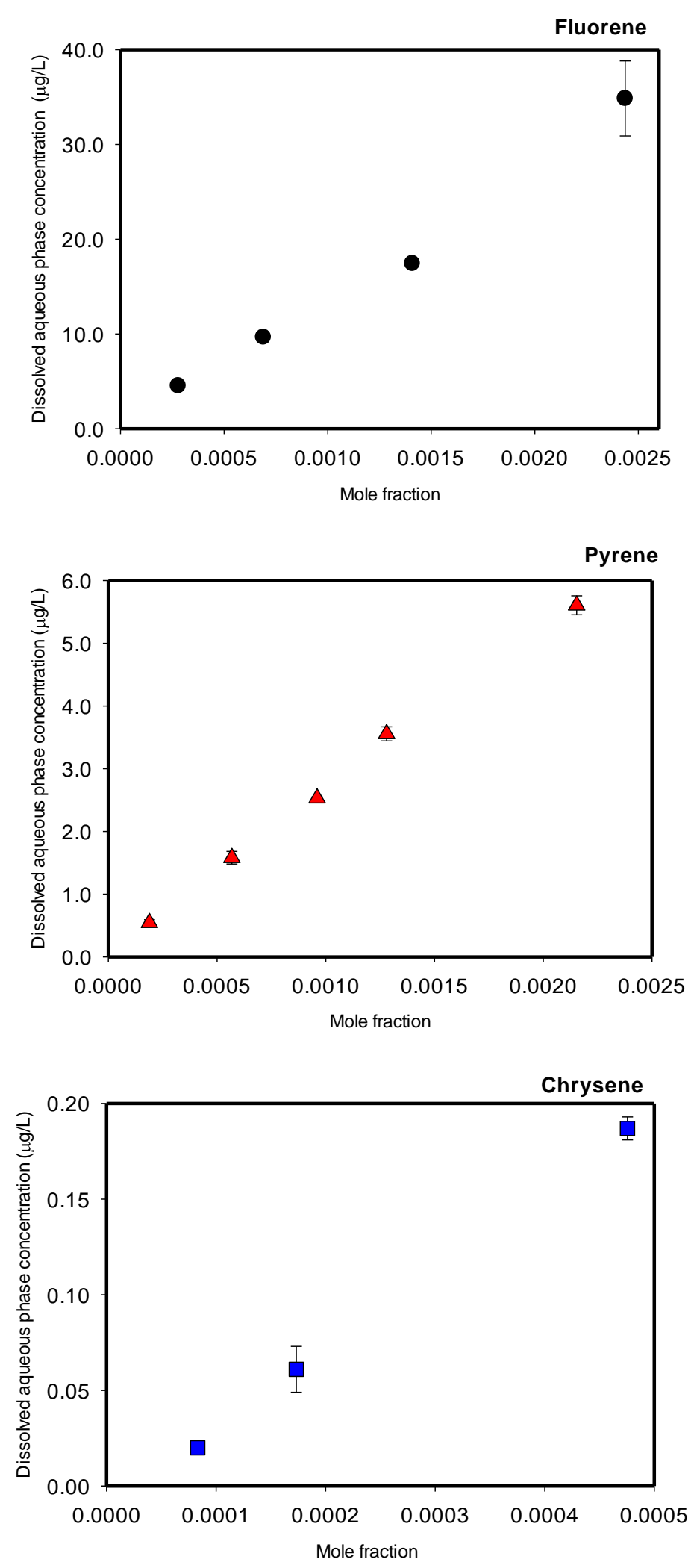

Figure 20: Dissolved aqueous phase concentrations versus increased mole fraction in the Alkane Oil (AO) for fluorene (top), pyrene (middle) and chrysene (bottom). 
Furthermore, using $10 \mu \mathrm{m}$ droplets lead to rapid dissolution and establishment of aqueous phase equilibrium between the dissolved and oil droplets as the percentages between the dispersions for each particular PAH were fairly consistent even when prepared at different times. This is in agreement with Mackay and Leinonen (1975) who demonstrated that dissolution from droplets smaller than $100 \mu \mathrm{m}$ was very rapid with dissolution rates highly dependent on the surface area of the droplets. Thus, dissolution from $10 \mu \mathrm{m}$ droplets with large surface areas such as the ones prepared in this study resulted in an almost instant equilibrium for the PAHs since they were within their solubility limit.

To corroborate that the difference in solubility of the target PAHs was solely based on their corresponding Kow value, the mole fraction of each compound was kept constant in the $\mathrm{AO}$ and their resulting dissolved aqueous phase concentrations were measured and compared as shown in Figure 21. As expected, the dissolved concentration (LogS) of the PAHs was linearly related to Log Kow confirming that partitioning between the water and droplet phase can be explained in terms of the PAH's Kow value. PAHs with low Kow will be less lipophilic and more soluble in water than higher Kow PAHs (Viaene et al., 2014).

As shown in this study, using micron-size droplets as a dosing platform was successful for the creation of exposure media containing individual PAHs at consistent and predictable concentrations. This delivery system is particularly useful for applications in the assessment of aquatic toxicology. Alternative methods used to introduce sparingly soluble compounds into aqueous solutions often use cosolvents such as methanol, ethanol or DMSO to modify the bioavailability of the compound in the test (Kwon and Kwon, 
2012). Kwon and Kwon (2012) found an increase in aqueous solubility for 8 specific PAHs with increase in small volume fraction (0.2-2\%) of 3 different cosolvents (DMSO, ethanol and acetone). Even though the mole fraction of the cosolvent is extremely small in comparison to the water, the use of carrier solvents for toxicant delivery can have potential confounding effects for toxicology tests. However, the micron-size droplets composed of n-alkanes which are insoluble in water would not increase the solubility of the PAHs in the aqueous phase, can be removed by filtration, and offer a more representative matrix for oil test studies.

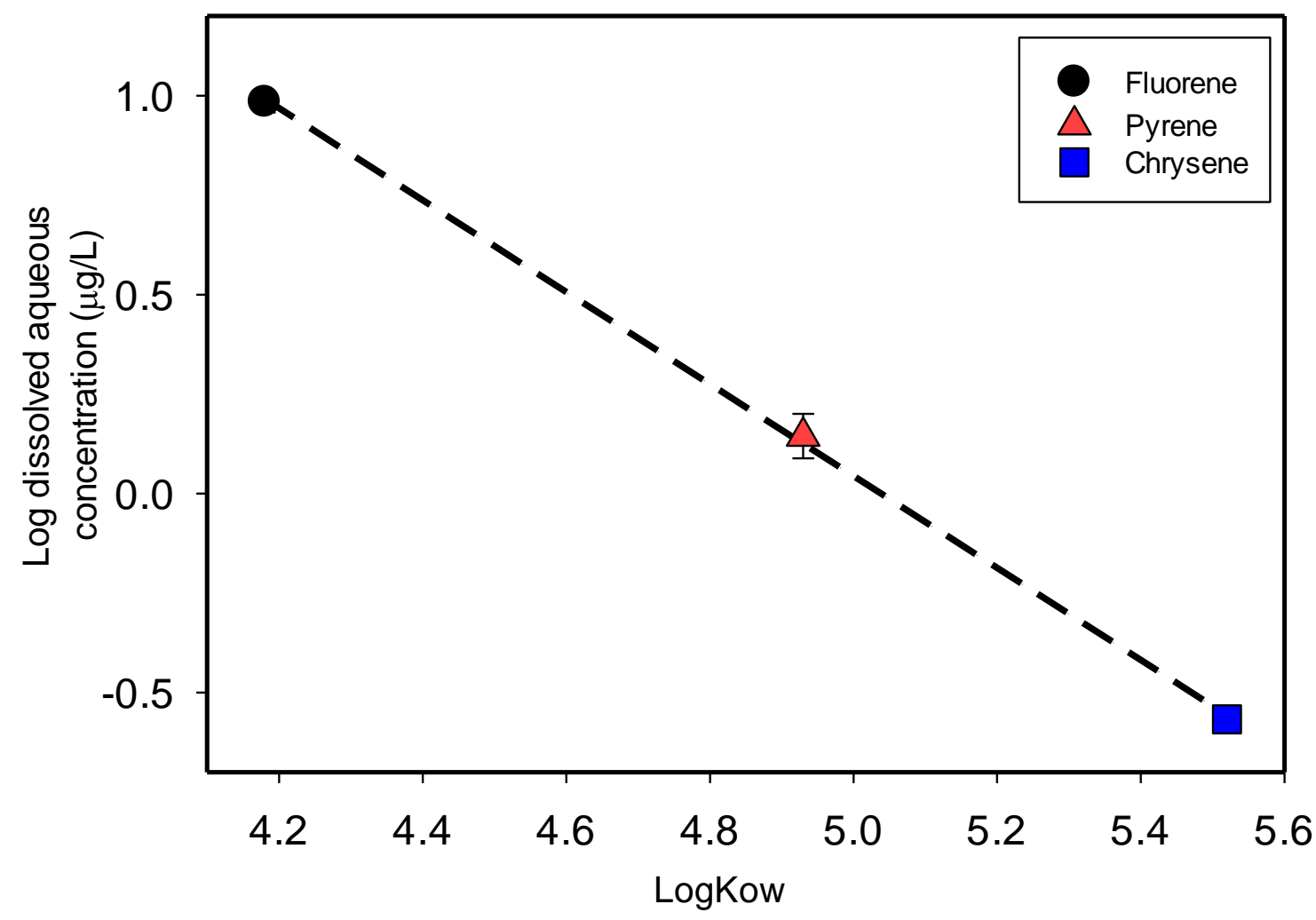

Figure 21: Comparison of $\log \mathrm{S}$ versus $\log$ Kow at constant mole fraction of the individual PAHs in the AO. 


\subsubsection{Characterization of PAH mixture-AO and a well-studied crude oil}

In order to further demonstrate the applicability of the described dosing system, the $\mathrm{AO}$ was mixed with 10 different PAHs at nominal concentrations that were similar to the ones found for the MC252 Macondo light sweet crude oil released during the deepwater horizon (DWH) incident (see section 3.2.1). The measured concentrations of the PAHs in both the AO and the Macondo oil were compared and found to agree well with each other. The detailed chemical composition of the PAHs is listed in appendix 3. Oil water dispersions (OWDs) and water soluble fractions (WSFs) were prepared with both oils and the concentrations of the PAHs measured in both the particulate and dissolved phase are presented in Figure 22. The results indicate that the majority of the PAHs showed similar concentrations in both the particulate and dissolved phase for both oils with the exception of naphthalene, C2-naphthalene and phenanthrene. The difference may arise from having slightly higher concentrations of those PAHs in the prepared AO

as opposed to the Macondo oil (appendix 3). 2,6-dimethylnaphthane was chosen to represent the family of $\mathrm{C} 2$-naphthalenes in the $\mathrm{AO}$, however the Macondo oil contains a wider range of di-methylated naphthalenes' such as 1,3-dimethylnaphthalene, 1,6dimethylnaphthalene, etc. Even though, 2,6-dimethylnaphthalene was added to the AO at concentrations similar to the measured concentration of C2-naphthalene in the Macondo oil, the contribution of the other di-methylated naphthalenes' in the Macondo oil could be a reason for the higher concentrations measured in both the particulate and dissolved phase. 

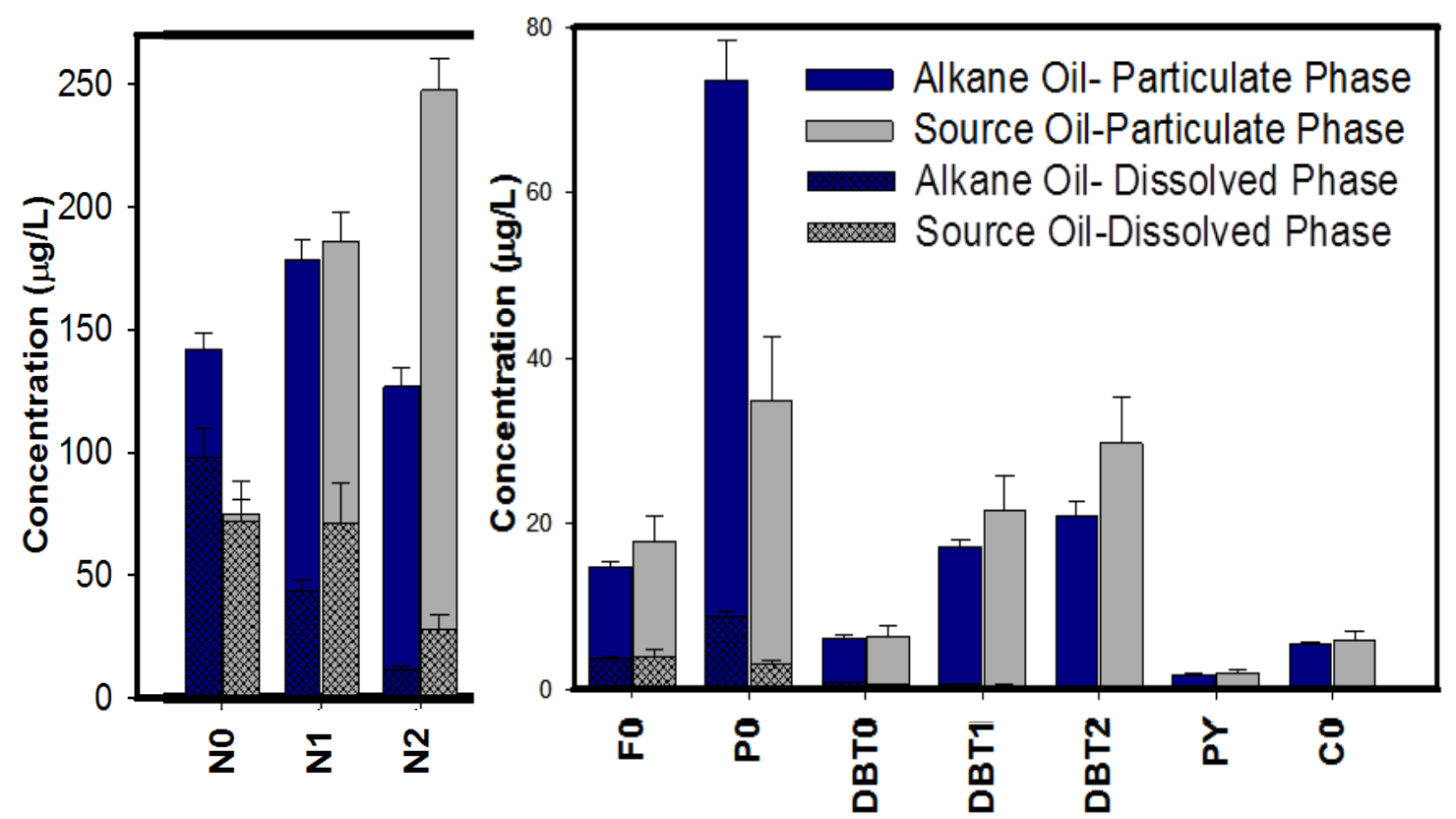

Figure 22: Distribution of the concentration of PAHs in both particulate and dissolved phase for the Macondo source oil and the alkane oil.

When assessing the potential risk of complex mixtures such as petroleum products, aquatic toxicity is often predicted from solubility models which use Raoult's law assuming ideal behavior to estimate the aqueous-phase concentrations of the different components present in the mixture (McGrath et al., 2005). Important parameters for the applicability of the solubility model include the proper determination of the physicochemical properties of the components present in the mixture which are often calculated from the SPARC model (Redman et al., 2012a). In addition, the average molecular weight $\left(\mathrm{MW}_{\mathrm{av}}\right)$ of a crude oil mixture is an essential parameter for the proper determination of the mole fraction of the individual constituents, thus the value needs to be accurate in order for a correct prediction of the dissolved phase concentrations (Kang et al., 2014). Chemical characterization is the limiting factor in obtaining an accurate $\mathrm{MW}_{\mathrm{av}}$ for crude oils and the $\mathrm{MW}_{\mathrm{av}}$ is often estimated by doing a best-fit of the empirical 
data to the predicted solubility (Kang et al., 2014). The implications of using either an estimated $\mathrm{MW}_{\mathrm{av}}$ or a best fit $\mathrm{MW}$ av may lead to erroneous prediction of the aqueous phase concentrations and interpretation of the exposure media data. The creation of the AO gives the opportunity to investigate the importance of the $\mathrm{MW}_{\mathrm{av}}$ value in the solubility model.

Because all the components in the PAH-AO mixture are known, a MW $\mathrm{Mv}_{\mathrm{av}}$ of 173 $\mathrm{g} / \mathrm{mol}$ for the $\mathrm{AO}$ was calculated and it was used to calculate the mole fractions used for predicting the aqueous phase concentrations based on Raoult's law. The measured aqueous phase concentrations of the PAHs in the WSFs prepared with the PAH-AO mixture was compared to the predicted aqueous phase concentrations and shown in Figure 23. The results indicate that the predicted values agree very well with the measured ones for the 2-ring and 3-ring PAHs within a factor of 2 with the exception of two PAHs (fluorene and phenanthrene). The discrepancy between the predicted and measured values for those particular PAHs may be due to errors in the prediction of the subcooled liquid solubility which is predicted from the SPARC model and can affect the predicted aqueous phase concentration. For example, Kang et al. (2014) reported an aqueous solubility of $0.82 \mathrm{mg} / \mathrm{L}$ for phenanthrene while Liu et al. (2013) reported an aqueous solubility of $1.18 \mathrm{mg} / \mathrm{L}$. Clearly, these parameters which are essential for the proper calculation of the predicted aqueous concentrations have to be further validated with experimental work. The large deviations for the 4-ring PAHs (pyrene and chrysene) shown in Figure 23 and 24 are likely due to the presence of stable droplets with sizes that were below the optical detection limit of the microscopy method (Redman et al., 2012a). 
The solubility model reported for a WAF preparation showed similar deviations for the larger molecular weight PAHs as observed in this results (Redman et al., 2012a).

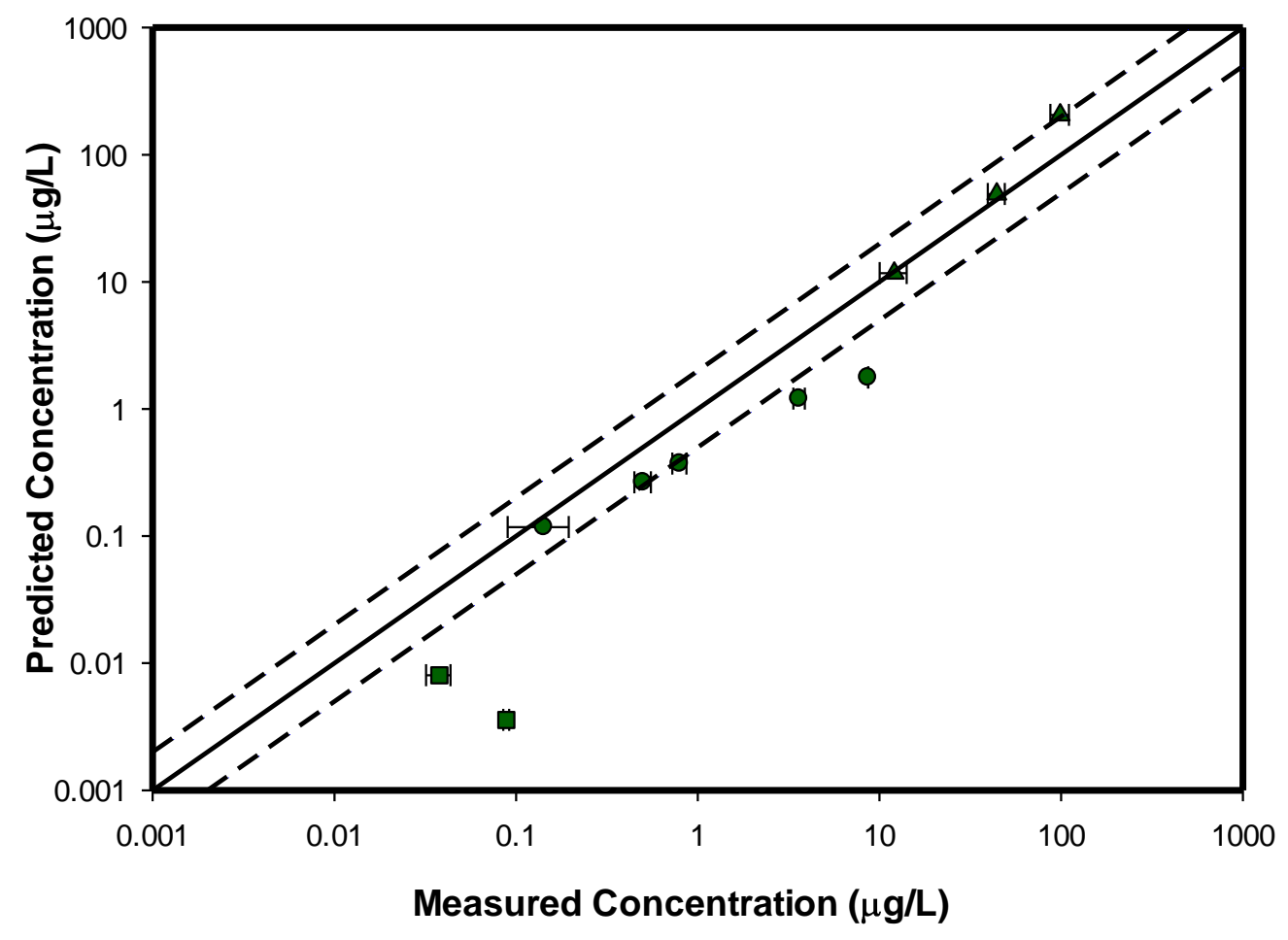

Figure 23: Predicted versus measured dissolved phase concentrations of PAHs present in the water-soluble fractions (WSFs) prepared with the Alkane Oil. The 2-ring aromatics are shown as $(\boldsymbol{\Delta})$, 3-ring PAHs $(\bullet)$ and 4-ring PAHs (๘). The solid and dashed lines indicate $1: 1$ and $2: 1$ or $1: 2$ lines, respectively.

Additionally, the solubility model was fitted with the data obtained for the WSF prepared with the Macondo oil. Since the $\mathrm{MW}_{\mathrm{av}}$ of the Macondo sweet light oil has not been reported in the literature, the predicted aqueous phase concentrations were calculated using an estimated $\mathrm{MW}_{\mathrm{av}}(187 \mathrm{~g} / \mathrm{mol})$ reported for a neat Alaskan North Slope oil (Redman et al., 2012a) which is shown as the 1:1 dashed line and a best fit MW av (272 $\mathrm{g} / \mathrm{mol}$ ) which is presented as the 1:1 solid line (Fig. 24). Clearly, using a $\mathrm{MW}_{\text {av }}$ of 187 
$\mathrm{g} / \mathrm{mol}$ for the Macondo oil underestimated the predicted values, as the majority of the values were not within the dashed line. The $\mathrm{MW}_{\mathrm{av}}$ of the Macondo oil was estimated by comparing the measured versus the predicted concentration of the PAHs so the predicted values would be more consisted with the measured concentrations. Using the estimated $\mathrm{MW}_{\mathrm{av}}$ of $272 \mathrm{~g} / \mathrm{mol}$ resulted in an increase fit for the model as the measured concentrations were closer to the 1:1 solid line (predicted values) with the exception of the high molecular weight components due to the possible presence of small droplets that were not detected by microscopy method.

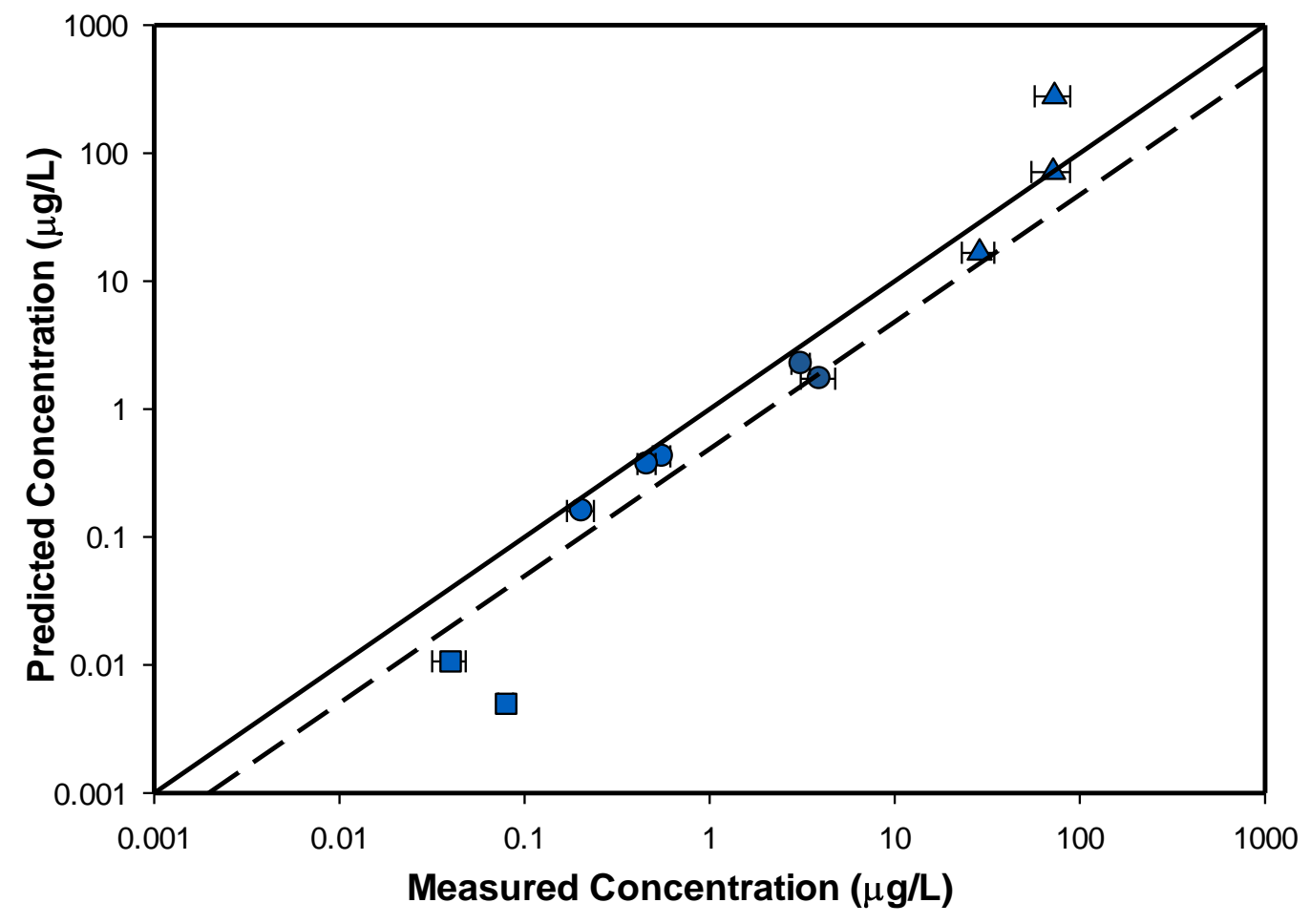

Figure 24: Predicted versus measured dissolved phase concentrations of PAHs measured from the water soluble fractions (WSFs) prepared with the Macondo source oil. The solid line indicate the 1:1 line calculated using the $\mathrm{MW}=187 \mathrm{~g} / \mathrm{mol}$ and the dashed line indicate the $1: 1$ line calculated using the best-fit $\mathrm{MW}=272 \mathrm{~g} / \mathrm{mol}$. 
The presented results demonstrated the usefulness of the synthetic Alkane Oil mixture to conduct experiments to simulate complex multicomponent PAHs that are often encounter in environmental settings. The advantage of using this synthetic PAH-AO mixture to represent petroleum products is been able to design and manipulate its exact chemical composition. Petroleum is a very complex mixture containing hundreds if not thousands of unknown compounds which makes it extremely difficult to fully characterize. Asphaltenes and resins are the heaviest constituents in crude oil and contain polar compounds that are highly soluble in water and are hard to identify and quantify (Speight, 2004). These polar components can significantly affect the mole fraction of the oil and they are often not taken into account when assessing toxicity.

In order to determine the toxicity from a chemical mixture, the concentrations of all the components in that mixture needs to be measured not only because each of the components can be a potential contributor to the total toxicity but also because they can affect the bioavailability of the other components. This is especially important when expressing toxicity in terms of toxic units (TU) as it has been demonstrated that the total toxicity from a mixture is additive and dependent on the aqueous concentration of each of the components in the mixture in equilibrium with the solution (Di Toro et al., 2007). The methodology described in this work is ideal to account for exposures to a mixture of hydrocarbons of known chemical composition and whose TU can be accurately predicted so results in toxicological studies can be more comparable and reproducible. 


\subsection{Conclusion}

The use of micron-size droplets prepared with a synthetic Alkane Oil containing individual and a mixture of PAHs was successfully developed and tested in order to deliver known aqueous concentrations of toxicants of concern into the aqueous media. This delivery system represents an innovative method for the successful establishment of rapid dissolution and equilibration of different compounds and can be used as a dosing platform for toxicology studies. Raoult's law was used to demonstrate that dissolution of PAHs from droplets into the water column is governed by the compound's mole fraction and aqueous solubility as an increase in molar fraction resulted in a proportional increase in the dissolved aqueous concentration. As expected, when the mole fraction of the individual PAHs in the $\mathrm{AO}$ was kept constant, the dissolved aqueous concentration only depended on the aqueous solubility of the individual PAH as described by the compounds $\log$ Kow.

In addition, the results also point out the importance of an accurate determination of $\mathrm{MW}_{\mathrm{av}}$ for the proper calculation of the predicted aqueous phase concentrations from complex mixture such as crude oils. This study is also the first to estimate the $\mathrm{MW}_{\text {av }}$ for the source Macondo oil (272 $\mathrm{g} / \mathrm{mol})$ which can be used by others to establish solubility plots. Clearly, this methodology has multiple applications in toxicological studies from assessing the toxicity of oil droplets alone or to determine the bioavailability of dissolved components. Future studies will focus on applying this method to study the photochemical evolution of individual PAHs from AO droplets. 


\section{Conclusion}

This research demonstrated how important and useful it is to conduct a comprehensive physical and chemical characterization of oil water mixtures used for toxicology testing. First, a combination of simple methods were developed and applied in order to characterize and quantify the droplet size distribution of the different oil water dispersions. UV-epi fluorescence microscopy offered solid evidence of the presence or absence of droplets and the coulter counter complemented the characterization of droplets to determine oil droplet distribution based on volume and to produce a semi-quantitative determination of oil concentration and loading verifications in real time. EEMs by fluorescence spectroscopy was also shown to be a reliable and effective method to get a quick estimation of concentration of bulk oil and to further establish the presence of droplets. Droplet size distribution was influenced by the method used to prepare the oil water mixtures and also by the type of oil and weathering degree. Larger droplets were formed with high energy preparations in comparison to the chemically-enhanced dispersions for the oil water mixtures prepared with the source oil. It was also demonstrated that droplet sizes were an important parameter for the stability of the dispersions, as oil water mixtures that contained smaller droplet sizes were significantly more stable.

Secondly, different oil water mixtures prepared by using well-established and new methodologies were compared based on their chemical composition and stability. More than 15 years ago, Singer et al. (2000) described a standardized protocol for the preparation of exposure media (WAFs and CEWAFs) so that toxicology data can be 
more comparable. The clear advantages of WAF preparations reside in the separation of the dissolved components of a complex environmental mixture as oil, their well-known behavior and preparation protocols and their accurate representation of bioavailable fractions of weathered materials through their environmental evolution. CEWAF preparations introduce additional confounding factors such as the presence of a chemical dispersant but are an appropriate representation of conditions encountered during spill response and cleanup efforts. Alternatively, new novel techniques for the preparation of OWDs using the pressurized fluid injection system were proven to be successful and may complement CEWAFs as the basic process to produce dispersions containing oil droplets. However, due to the higher viscosity of weathered oils and the loss of dispersibility, additional energy is required to produce oil-water dispersions containing entrained insoluble portions of the test material. The high-energy mixing methods (HEWAFs and SHEWAFs) using standardized mixing equipment were proven useful for the preparation of oil water dispersion with weathered oils. However, it was demonstrated that at typical loading ratios of 1:1000 $(1 \mathrm{~g} / \mathrm{L})$ all high energy preparations were unstable and suffered from significant losses during the first 24 hour unless the oil loadings were significantly reduced and thus they need to be carefully monitored when used in toxicology studies.

Lastly, an Alkane Oil was used as a surrogate of an actual oil in order to prepare oil water dispersions with consistent droplet sizes. The composition of the alkane oil was manipulated by adding single and mixed PAHs at different concentrations in order to study their dissolution from micron-size droplets. The characterization of oil droplets containing increasing amounts of pyrene showed an increase in fluorescence in the droplet phase by examination in both the epi-fluorescence microscope and EEMs 
obtained by the spectrofluoremeter. The dissolution of PAHs from the droplets was governed by raoult's law as predicted and was highly dependent on the mole fraction and solubility of the PAHs (described in terms of its Kow). This dosing mechanism was proven successful for the generation of oil water dispersions and could be used to produce exposure media that could isolate the potential contributions of physical and chemical effects as well as target the specific appropriate mechanisms of actions exerted by PAHs. 


\section{REFERENCES}

Almeida, J. R., C. Gravato, and L. Guilhermino, 2012, Challenges in assessing the toxic effects of polycyclic aromatic hydrocarbons to marine organisms: A case study on the acute toxicity of pyrene to the European seabass (Dicentrarchus labrax L.): Chemosphere, v. 86, p. 926-937.

Anderson, J. W., 1985, Toxicity of dispersed and undispersed Prudhoe Bay crude oil fractions to shrimp, fish, and their larvae, American Petroleum Institute.

Anderson, J. W., R. Riley, S. Kiesser, and J. Gurtisen, 1987, TOXICITY OF DISPERSED AND UNDISPERSED PRUDHOE BAY CRUDE OIL FRACTIONS TO SHRIMP AND FISH: International Oil Spill Conference Proceedings, v. 1987, p. 235-240.

Atlas, R. M., and T. C. Hazen, 2011, Oil Biodegradation and Bioremediation: A Tale of the Two Worst Spills in US History: Environmental Science \& Technology, v. 45, p. 6709-6715.

Aurand, D., and G. Coelho, 2005, Cooperative aquatic toxicity testing of dispersed oil and the chemical response to oil spills: Ecological Effects Research Forum (CROSERF): Inc. Lusby, MD. Tech. Report, p. 07-03.

Baelum, J., S. Borglin, R. Chakraborty, J. L. Fortney, R. Lamendella, O. U. Mason, M. Auer, M. Zemla, M. Bill, M. E. Conrad, S. A. Malfatti, S. G. Tringe, H. Y. Holman, T. C. Hazen, and J. K. Jansson, 2012, Deep-sea bacteria enriched by oil and dispersant from the Deepwater Horizon spill: Environmental Microbiology, v. 14, p. 2405-2416.

Barron, M. G., and L. Ka'aihue, 2003, Critical evaluation of CROSERF test methods for oil dispersant toxicity testing under subarctic conditions: Marine Pollution Bulletin, v. 46, p. 1191-1199.

Bejarano, A. C., J. R. Clark, and G. M. Coelho, 2014, Issues and challenges with oil toxicity data and implications for their use in decision making: A quantitative review: Environmental Toxicology and Chemistry, v. 33, p. 732-742.

Bobra, A. M., W. Y. Shiu, D. Mackay, and R. H. Goodman, 1989, Acute toxicity of dispersed fresh and weathered crude oil and dispersants to Daphnia magna: Chemosphere, v. 19, p. 1199-1222. 
Boehm, P. D., G. S. Douglas, W. A. Burns, P. J. Mankiewicz, D. S. Page, and A. E. Bence, 1997, Application of petroleum hydrocarbon chemical fingerprinting and allocation techniques after the Exxon Valdez oil spill: Marine Pollution Bulletin, v. 34, p. 599-613.

BP, 2014, "Gulf Science Data Water Chemistry Data File.” Reference No. W-01v01-01. Last modified August 2014. http://gulfsciencedata.bp.com/go/doc/6145/1942326/.

Bragg, J. R., R. C. Prince, E. J. Harner, and R. M. Atlas, 1994, Effectiveness of bioremediation for the Exxon Valdez oil spill.

Brakstad, O. G., T. Nordtug, and M. Throne-Hoist, 2015, Biodegradation of dispersed Macondo oil in seawater at low temperature and different oil droplet sizes: Marine Pollution Bulletin, v. 93, p. 144-152.

Brette, F., B. Machado, C. Cros, J. P. Incardona, N. L. Scholz, and B. A. Block, 2014, Crude Oil Impairs Cardiac Excitation-Contraction Coupling in Fish: Science, v. 343, p. $772-776$.

Brewton, R. A., R. Fulford, and R. J. Griffitt, 2013, Gene Expression and Growth as Indicators of Effects of the BP Deepwater Horizon Oil Spill on Spotted Seatrout (Cynoscion nebulosus): Journal of Toxicology and Environmental Health, Part A, v. 76, p. 1198-1209.

Brown, R. S., P. Akhtar, J. Akerman, L. Hampel, I. S. Kozin, L. A. Villerius, and H. J. C. Klamer, 2001, Partition controlled delivery of hydrophobic substances in toxicity tests using poly(dimethylsiloxane) (PDMS) films: Environmental Science \& Technology, v. 35, p. 4097-4102.

Bugden, J. B. C., C. W. Yeung, P. E. Kepkay, and K. Lee, 2008, Application of ultraviolet fluorometry and excitation-emission matrix spectroscopy (EEMS) to fingerprint oil and chemically dispersed oil in seawater: Marine Pollution Bulletin, v. 56, p. 677-685.

Cachada, A., R. Pereira, E. F. da Silva, and A. C. Duarte, 2014, The prediction of PAHs bioavailability in soils using chemical methods: State of the art and future challenges: Science of the Total Environment, v. 472, p. 463-480.

Carls, M. G., L. Holland, M. Larsen, T. K. Collier, N. L. Scholz, and J. P. Incardona, 2008, Fish embryos are damaged by dissolved PAHs, not oil particles: Aquatic Toxicology, v. 88, p. 121-127. 
Chase, D. A., D. S. Edwards, G. Q. Qin, M. R. Wages, M. M. Willming, T. A. Anderson, and J. D. Maul, 2013, Bioaccumulation of petroleum hydrocarbons in fiddler crabs (Uca minax) exposed to weathered MC-252 crude oil alone and in mixture with an oil dispersant: Science of the Total Environment, v. 444, p. 121-127.

Coelho, G., J. Clark, and D. Aurand, 2013, Toxicity testing of dispersed oil requires adherence to standardized protocols to assess potential real world effects: Environmental Pollution, v. 177, p. 185-188.

Conmy, R. N., P. G. Coble, J. Farr, A. M. Wood, K. Lee, W. S. Pegau, I. D. Walsh, C. R. Koch, M. I. Abercrombie, M. S. Miles, M. R. Lewis, S. A. Ryan, B. J. Robinson, T. L. King, C. R. Kelble, and J. Lacoste, 2014, Submersible Optical Sensors Exposed to Chemically Dispersed Crude Oil: Wave Tank Simulations for Improved Oil Spill Monitoring: Environmental Science \& Technology, v. 48, p. 1803-1810.

Couillard, C. M., K. Lee, B. Legare, and T. L. King, 2005, Effect of dispersant on the composition of the water-accommodated fraction of crude oil and its toxicity to larval marine fish: Environmental Toxicology and Chemistry, v. 24, p. 1496-1504.

Delvigne, G. A. L., 1987, Droplet size distribution of naturally dispersed oil, Fate and effects of oil in marine ecosystems, Springer, p. 29-40.

Di Toro, D. M., and J. A. McGrath, 2000, Technical basis for narcotic chemicals and polycyclic aromatic hydrocarbon criteria. II. Mixtures and sediments: Environmental Toxicology and Chemistry, v. 19, p. 1971-1982.

Di Toro, D. M., J. A. McGrath, and W. A. Stubblefield, 2007, Predicting the toxicity of neat and weathered crude oil: Toxic potential and the toxicity of saturated mixtures: Environmental Toxicology and Chemistry, v. 26, p. 24-36.

Echols, B. S., A. J. Smith, P. R. Gardinali, and G. M. Rand, 2015, Acute aquatic toxicity studies of Gulf of Mexico water samples collected following the Deepwater Horizon incident (May 12, 2010 to December 11, 2010): Chemosphere, v. 120, p. 131-137.

Engraff, M., C. Solere, K. E. C. Smith, P. Mayer, and I. Dahllof, 2011, Aquatic toxicity of PAHs and PAH mixtures at saturation to benthic amphipods: Linking toxic effects to chemical activity: Aquatic Toxicology, v. 102, p. 142-149.

Faksness, L. G., D. Altin, T. Nordtug, P. S. Daling, and B. H. Hansen, 2015, Chemical comparison and acute toxicity of water accommodated fraction (WAF) of source 
and field collected Macondo oils from the Deepwater Horizon spill: Marine Pollution Bulletin, v. 91, p. 222-229.

Farrington, J. W., 2014, Oil Pollution in the Marine Environment II: Fates and Effects of Oil Spills: Environment: Science and Policy for Sustainable Development, v. 56, p. 16-31.

Franklin, F. L., and R. Lloyd, 1986, The relationship between oil droplet size and the toxicity of dispersant/oil mixtures in the standard MAFF 'sea' test: Oil and Chemical Pollution, v. 3, p. 37-52.

French-McCay, D. P., 2002, Development and application of an oil toxicity and exposure model, OilToxEx: Environmental Toxicology and Chemistry, v. 21, p. 2080-2094.

Gauthier, P. T., W. P. Norwood, E. E. Prepas, and G. G. Pyle, 2014, Metal-PAH mixtures in the aquatic environment: A review of co-toxic mechanisms leading to morethan-additive outcomes: Aquatic Toxicology, v. 154, p. 253-269.

González-Doncel, M., L. González, C. Fernández-Torija, J. M. Navas, and J. V. Tarazona, 2008, Toxic effects of an oil spill on fish early life stages may not be exclusively associated to PAHs: Studies with Prestige oil and medaka (Oryzias latipes): Aquatic Toxicology, v. 87, p. 280-288.

Hansen, B. H., D. Altin, A. J. Olsen, and T. Nordtug, 2012, Acute toxicity of naturally and chemically dispersed oil on the filter-feeding copepod Calanus finmarchicus: Ecotoxicology and Environmental Safety, v. 86, p. 38-46.

Hansen, B. H., T. Nordtug, D. Altin, A. Booth, K. M. Hessen, and A. J. Olsen, 2009, Gene Expression of GST and CYP330A1 in Lipid-Rich and Lipid-Poor Female Calanus finmarchicus (Copepoda: Crustacea) Exposed to Dispersed Oil: Journal of Toxicology and Environmental Health-Part a-Current Issues, v. 72, p. 131-139.

Hazen, T. C., E. A. Dubinsky, T. Z. DeSantis, G. L. Andersen, Y. M. Piceno, N. Singh, J. K. Jansson, A. Probst, S. E. Borglin, J. L. Fortney, W. T. Stringfellow, M. Bill, M. E. Conrad, L. M. Tom, K. L. Chavarria, T. R. Alusi, R. Lamendella, D. C. Joyner, C. Spier, J. Baelum, M. Auer, M. L. Zemla, R. Chakraborty, E. L. Sonnenthal, P. D'Haeseleer, H. Y. N. Holman, S. Osman, Z. M. Lu, J. D. Van Nostrand, Y. Deng, J. Z. Zhou, and O. U. Mason, 2010, Deep-Sea Oil Plume Enriches Indigenous OilDegrading Bacteria: Science, v. 330, p. 204-208.

Incardona, J. P., L. D. Gardner, T. L. Linbo, T. L. Brown, A. J. Esbaugh, E. M. Mager, J. D. Stieglitz, B. L. French, J. S. Labenia, C. A. Laetz, M. Tagal, C. A. Sloan, A. 
Elizur, D. D. Benetti, M. Grosell, B. A. Block, and N. L. Scholz, 2014, Deepwater Horizon crude oil impacts the developing hearts of large predatory pelagic fish: Proceedings of the National Academy of Sciences, v. 111, p. E1510-E1518.

Incardona, J. P., T. L. Swarts, R. C. Edmunds, T. L. Linbo, A. Aquilina-Beck, C. A. Sloan, L. D. Gardner, B. A. Block, and N. L. Scholz, 2013, Exxon Valdez to Deepwater Horizon: Comparable toxicity of both crude oils to fish early life stages: Aquatic Toxicology, v. 142, p. 303-316.

Jung, J.-H., C. E. Hicken, D. Boyd, B. F. Anulacion, M. G. Carls, W. J. Shim, and J. P. Incardona, 2013, Geologically distinct crude oils cause a common cardiotoxicity syndrome in developing zebrafish: Chemosphere, v. 91, p. 1146-1155.

Kang, H.-J., S.-Y. Lee, J.-Y. Roh, U. H. Yim, W. J. Shim, and J.-H. Kwon, 2014, Prediction of Ecotoxicity of Heavy Crude Oil: Contribution of Measured Components: Environmental Science \& Technology, v. 48, p. 2962-2970.

Kopecka-Pilarczyk, J., and A. D. Correia, 2009, Biochemical response in gilthead seabream (Sparus aurata) to in vivo exposure to a mix of selected PAHs: Ecotoxicology and Environmental Safety, v. 72, p. 1296-1302.

Kwon, H. C., and J. H. Kwon, 2012, Measuring Aqueous Solubility in the Presence of Small Cosolvent Volume Fractions by Passive Dosing: Environmental Science \& Technology, v. 46, p. 12550-12556.

Landrum, P. F., G. R. Lotufo, D. C. Gossiaux, M. L. Gedeon, and J.-H. Lee, 2003, Bioaccumulation and critical body residue of PAHs in the amphipod, Diporeia spp.: additional evidence to support toxicity additivity for PAH mixtures: Chemosphere, v. 51, p. 481-489.

Lehr, W., S. Bristol, and A. Possolo, 2010, Oil Budget Calculator Deepwater Horizon Technical Documentation, Oil Budget Calculator Science and Engineering Team Report to the National Incident Command Interagency Solutions Group.

Less, S., and R. Vilagines, 2013, Light beam reflectance measurement of droplets diameter distribution in crude oil emulsions: Fuel, v. 109, p. 542-550.

Levinson, J., C. Sluszny, Y. Yasman, V. Bulatov, and I. Schechter, 2005, Detector for particulate polycyclic aromatic hydrocarbons in water: Analytical and Bioanalytical Chemistry, v. 381, p. 1584-1591. 
Li, Z., K. Lee, P. E. Kepkey, O. Mikkelsen, and C. Pottsmith, 2011, Monitoring Dispersed Oil Droplet Size Distribution at the Gulf of Mexico Deepwater Horizon Spill Site: International Oil Spill Conference Proceedings, v. 2011, p. abs377.

Li, Z., K. Lee, T. King, M. C. Boufadel, and A. D. Venosa, 2009, Evaluating Chemical Dispersant Efficacy in an Experimental Wave Tank: 2-Significant Factors Determining In Situ Oil Droplet Size Distribution: Environmental Engineering Science, v. 26, p. 1407-1418.

Liu, L., F. Wu, S. Haderlein, and P. Grathwohl, 2013, Determination of the subcooled liquid solubilities of PAHs in partitioning batch experiments: Geoscience Frontiers, v. 4, p. 123-126.

Lyons, W. C., and G. J. Plisga, 2011, Standard handbook of petroleum and natural gas engineering, Gulf Professional Publishing.

MacKay, D., and P. Leinonen, 1975, Mathematical model of the behavior of oil spills on water with natural and chemical dispersion, Environment Protection Service.

Mager, E. M., A. J. Esbaugh, J. D. Stieglitz, R. Hoenig, C. Bodinier, J. P. Incardona, N. L. Scholz, D. D. Benetti, and M. Grosell, 2014, Acute Embryonic or Juvenile Exposure to Deepwater Horizon Crude Oil Impairs the Swimming Performance of Mahi-Mahi (Coryphaena hippurus): Environmental Science \& Technology, v. 48, p. 7053-7061.

Manzetti, S., 2012, Ecotoxicity of polycyclic aromatic hydrocarbons, aromatic amines, and nitroarenes through molecular properties: Environmental Chemistry Letters, v. 10, p. 349-361.

Marshall, A. G., and R. P. Rodgers, 2004, Petroleomics: The Next Grand Challenge for Chemical Analysis: Accounts of Chemical Research, v. 37, p. 53-59.

McAuliffe, C. D., J. C. Johnson, S. H. Greene, G. P. Canevari, and T. D. Searl, 1980, Dispersion and weathering of chemically treated crude oils on the ocean: Environmental Science \& Technology, v. 14, p. 1509-1518.

McGrath, J. A., and D. M. Di Toro, 2009, Validation of the target lipid model for toxicity assessment of residual petroleum constituents: Monocyclic and polycyclic aromatic hydrocarbons: Environmental Toxicology and Chemistry, v. 28, p. 11301148 . 
McGrath, J. A., T. E. Parkerton, F. L. Hellweger, and D. M. Di Toro, 2005, Validation of the narcosis target lipid model for petroleum products: Gasoline as a case study: Environmental Toxicology and Chemistry, v. 24, p. 2382-2394.

Neff, J., 1988, Composition and fate of petroleum and spill-treating agents in the marine environment: SYNTHESIS OF EFFECTS OF OIL ON MARINE MAMMALS.

Nisbet, I. C. T., and P. K. Lagoy, 1992, TOXIC EQUIVALENCY FACTORS (TEFS) FOR POLYCYCLIC AROMATIC-HYDROCARBONS (PAHS): Regulatory Toxicology and Pharmacology, v. 16, p. 290-300.

Nordtug, T., A. J. Olsen, D. Altin, S. Meier, I. Overrein, B. H. Hansen, and O. Johansen, 2011a, Method for generating parameterized ecotoxicity data of dispersed oil for use in environmental modelling: Marine Pollution Bulletin, v. 62, p. 2106-2113.

Nordtug, T., A. J. Olsen, D. Altin, I. Overrein, W. Storoy, B. H. Hansen, and F. De Laender, 2011b, Oil droplets do not affect assimilation and survival probability of first feeding larvae of North-East Arctic cod: Science of the Total Environment, v. 412, p. 148-153.

Nordtug, T., A. J. Olsen, I. Salaberria, I. B. Øverjordet, D. Altin, I. F. Størdal, and B. H. Hansen, 2015, Oil droplet ingestion and oil fouling in the copepod Calanus finmarchicus exposed to mechanically and chemically dispersed crude oil: Environmental Toxicology and Chemistry, p. n/a-n/a.

North, Elizabeth, and W., 2011, Simulating Oil Droplet Dispersal From the Deepwater Horizon spill With a Lagrangian Approach: Geophysical Monograph Series 195, p. 217-226.

NRC, 2005, Understanding Oil Spill Dispersants: Efficacy and Effects, Washington, D.C., National Academy of Sciences.

Poulsen, M., L. Lemon, and J. F. Barker, 1992, DISSOLUTION OF MONOAROMATIC HYDROCARBONS INTO GROUNDWATER FROM GASOLINE OXYGENATE MIXTURES: Environmental Science \& Technology, v. 26, p. 2483-2489.

Rabodonirina, S., S. Net, B. Ouddane, D. Merhaby, D. Dumoulin, T. Popescu, and P. Ravelonandro, 2015, Distribution of persistent organic pollutants (PAHs, MePAHs, PCBs) in dissolved, particulate and sedimentary phases in freshwater systems: Environmental Pollution, v. 206, p. 38-48. 
Radovic, J. R., C. Aeppli, R. K. Nelson, N. Jimenez, C. M. Reddy, J. M. Bayona, and J. Albaiges, 2014, Assessment of photochemical processes in marine oil spill fingerprinting: Marine Pollution Bulletin, v. 79, p. 268-277.

Ramachandran, S., A. P. Quist, S. Kumar, and R. Lal, 2006, Cisplatin nanoliposomes for cancer therapy: AFM and fluorescence Imaging of cisplatin encapsulation, stability, cellular uptake, and toxicity: Langmuir, v. 22, p. 8156-8162.

Ramachandran, S. D., P. V. Hodson, C. W. Khan, and K. Lee, 2004a, Oil dispersant increases PAH uptake by fish exposed to crude oil: Ecotoxicology and Environmental Safety, v. 59, p. 300-308.

Ramachandran, S. D., C. W. Khan, P. V. Hodson, K. Lee, and T. King, 2004b, Role of droplets in promoting uptake of PAHs by fish exposed to chemically dispersed crude oil.

Ramirez, C. E., C. T. Wang, and P. R. Gardinali, 2014, Fully automated trace level determination of parent and alkylated PAHs in environmental waters by online SPE-LC-APPI-MS/MS: Analytical and Bioanalytical Chemistry, v. 406, p. 329344.

Reddy, C. M., J. S. Arey, J. S. Seewald, S. P. Sylva, K. L. Lemkau, R. K. Nelson, C. A. Carmichael, C. P. McIntyre, J. Fenwick, G. T. Ventura, B. A. S. Van Mooy, and R. Camilli, 2012, Composition and fate of gas and oil released to the water column during the Deepwater Horizon oil spill: Proceedings of the National Academy of Sciences of the United States of America, v. 109, p. 20229-20234.

Redman, A. D., 2015, Role of entrained droplet oil on the bioavailability of petroleum substances in aqueous exposures: Marine Pollution Bulletin, v. 97, p. 342-348.

Redman, A. D., J. A. McGrath, W. A. Stubblefield, A. Maki, and D. M. Di Toro, 2012a, Quantifying the concentration of crude oil microdroplets in oil-water preparations: Environmental Toxicology and Chemistry, v. 31, p. 1814-1822.

Redman, A. D., T. F. Parkerton, J. A. McGrath, and D. M. Di Toro, 2012b, PETROTOX: An aquatic toxicity model for petroleum substances: Environmental Toxicology and Chemistry, v. 31, p. 2498-2506.

Ryerson, T. B., R. Camilli, J. D. Kessler, E. B. Kujawinski, C. M. Reddy, D. L. Valentine, E. Atlas, D. R. Blake, J. de Gouw, S. Meinardi, D. D. Parrish, J. Peischl, J. S. Seewald, and C. Warneke, 2012, Chemical data quantify Deepwater Horizon hydrocarbon flow rate and environmental distribution: Proceedings of the 
National Academy of Sciences of the United States of America, v. 109, p. 2024620253.

Singer, M. M., D. Aurand, G. E. Bragin, J. R. Clark, G. M. Coelho, M. L. Sowby, and R. S. Tjeerdema, 2000, Standardization of the Preparation and Quantitation of Water-accommodated Fractions of Petroleum for Toxicity Testing: Marine Pollution Bulletin, v. 40, p. 1007-1016.

Smith, K. E. C., S. N. Schmicdt, N. Dom, R. Blust, M. Holmstrup, and P. Mayer, 2013, Baseline Toxic Mixtures of Non-Toxic Chemicals: "Solubility Addition" Increases Exposure for Solid Hydrophobic Chemicals: Environmental Science \& Technology, v. 47, p. 2026-2033.

Sorhus, E., R. B. Edvardsen, O. Karlsen, T. Nordtug, T. van der Meeren, A. Thorsen, C. Harman, S. Jentoft, and S. Meier, 2015, Unexpected Interaction with Dispersed Crude Oil Droplets Drives Severe Toxicity in Atlantic Haddock Embryos: Plos One, v. 10.

Speight, J. G., 2004, Petroleum asphaltenes - Part 1 - Asphaltenes, resins and the structure of petroleum: Oil \& Gas Science and Technology-Revue D Ifp Energies Nouvelles, v. 59, p. 467-477.

Spier, C., W. T. Stringfellow, T. C. Hazen, and M. Conrad, 2013, Distribution of hydrocarbons released during the $2010 \mathrm{MC} 252$ oil spill in deep offshore waters: Environmental Pollution, v. 173, p. 224-230.

Sterling, M. C., J. S. Bonner, C. A. Page, C. B. Fuller, A. N. S. Ernest, and R. L. Autenrieth, 2003, Partitioning of crude oil polycyclic aromatic hydrocarbons in aquatic systems: Environmental Science \& Technology, v. 37, p. 4429-4434.

Thibodeaux, L. J., K. T. Valsaraj, V. T. John, K. D. Papadopoulos, L. R. Pratt, and N. S. Pesika, 2011, Marine Oil Fate: Knowledge Gaps, Basic Research, and Development Needs; A Perspective Based on the Deepwater Horizon Spill: Environmental Engineering Science, v. 28, p. 87-93.

Tiruta-Barna, L., B. Mahjoub, L. Faure, K. Hanna, R. Bayard, and R. Gourdon, 2006, Assessment of the multi-compound non-equilibrium dissolution behaviour of a coal tar containing PAHs and phenols into water: Journal of Hazardous Materials, v. 132 , p. 277-286.

Tissot, B. P., and D. H. Welte, 1984, Petroleum formation and occurrence: Other Information: From review by Edward A. Beaumont, in The American Association 
of Petroleum Geologists Bulletin, Vol. 71, No. 4 (April 1987), Medium: X; Size: Pages: 699 p.

Trudel, K., R. C. Belore, J. V. Mullin, and A. Guarino, 2010, Oil viscosity limitation on dispersibility of crude oil under simulated at-sea conditions in a large wave tank: Marine Pollution Bulletin, v. 60, p. 1606-1614.

Viaene, K. P. J., C. R. Janssen, L. de Hoop, A. J. Hendriks, and F. De Laender, 2014, Evaluating the contribution of ingested oil droplets to the bioaccumulation of oil components - A modeling approach: Science of The Total Environment, v. 499, p. $99-106$.

Xie, Y. J., Y. Zhu, F. L. Xu, X. L. Liu, B. Xue, and S. Tao, 2007, A method for determining pyrene in mucus using synchronous fluorimetry with multiple standard additions: Chemosphere, v. 66, p. 1878-1883.

Yapa, P. D., M. R. Wimalaratne, A. L. Dissanayake, and J. A. DeGraff, 2012, How does oil and gas behave when released in deepwater?: Journal of Hydro-Environment Research, v. 6, p. 275-285.

Yim, U. H., S. Y. Ha, J. G. An, J. H. Won, G. M. Han, S. H. Hong, M. Kim, J.-H. Jung, and W. J. Shim, 2011, Fingerprint and weathering characteristics of stranded oils after the Hebei Spirit oil spill: Journal of Hazardous Materials, v. 197, p. 60-69.

Zhang, Y., Y. X. Zhu, K. K. Kwon, J. H. Park, and S. J. Kim, 2004, Novel method for determining pyrene biodegradation using synchronous fluorimetry: Chemosphere, v. 55, p. 389-394.

Zhong, Z., and F. You, 2011, Oil spill response planning with consideration of physicochemical evolution of the oil slick: A multiobjective optimization approach: Computers \& Chemical Engineering, v. 35, p. 1614-1630.

Zhou, Z. Z., L. D. Guo, A. M. Shiller, S. E. Lohrenz, V. L. Asper, and C. L. Osburn, 2013, Characterization of oil components from the Deepwater Horizon oil spill in the Gulf of Mexico using fluorescence EEM and PARAFAC techniques: Marine Chemistry, v. 148, p. 10-21. 


\section{APPENDICES}

Appendix 1: Abbreviations of PAHs

\begin{tabular}{|c|c|c|c|c|c|}
\hline Group & Compound & Abb. & Group & Compound & Abb. \\
\hline \multirow[t]{23}{*}{ 2-3 Ring PAHs } & Naphthalene & N0 & 4-6 Ring PAHs & Fluoranthene & FL0 \\
\hline & C1-Naphthalenes & $\mathrm{N} 1$ & & Pyrene & PY0 \\
\hline & C2-Naphthalenes & $\mathrm{N} 2$ & & C1-Fluoranthene/Pyrene & FL1 \\
\hline & C3-Naphthalenes & N3 & & C2-Fluoranthene/Pyrene & FL2 \\
\hline & C4-Naphthalenes & $\mathrm{N} 4$ & & C3-Fluoranthene/Pyrene & FL3 \\
\hline & Biphenyl & $\mathrm{BPH}$ & & C4-Fluoranthene/Pyrene & FL4 \\
\hline & Acenaphthylene & AY & & Benzo[a]Anthracene & BAA \\
\hline & Acenaphthene & $\mathrm{AE}$ & & Chrysene/Triphenylene & $\mathrm{C} 0$ \\
\hline & Fluorene & F0 & & C1-Chrysenes & $\mathrm{C} 1$ \\
\hline & C1-Fluorenes & F1 & & C2-Chrysenes & $\mathrm{C} 2$ \\
\hline & C2-Fluorenes & $\mathrm{F} 2$ & & C3-Chrysenes & $\mathrm{C} 3$ \\
\hline & C3- Fluorenes & F3 & & C4-Chrysenes & $\mathrm{C} 4$ \\
\hline & Anthracene & AN & & Benzo[b/j]fluoranthene & $\mathrm{BBF}$ \\
\hline & Phenanthrene & P0 & & Benzo[k]fluoranthene & BJKF \\
\hline & C1- Phenanthrene/anthracenes & $\mathrm{P} 1$ & & Benzo[e]pyrene & BEP \\
\hline & C2- Phenanthrene/anthracenes & $\mathrm{P} 2$ & & Benzo[a]pyrene & BAP \\
\hline & C3- Phenanthrene/anthracenes & P3 & & Perylene & PER \\
\hline & C4- Phenanthrene/anthracenes & $\mathrm{P} 4$ & & Indeno[1,2,3,-c,d]pyrene & IDP \\
\hline & Dibenzothiophene & DBT0 & & Dibenzo[a,h]anthracene & DBA \\
\hline & C1-Dibenzothiophenes & DBT1 & & Benzo[g,h,i] perylene & $\mathrm{BZP}$ \\
\hline & C2-Dibenzothiophenes & DBT2 & Other PAHs & Carbazole & CAR \\
\hline & C3-Dibenzothiophenes & DBT3 & & & \\
\hline & C4-Dibenzothiophene & DBT4 & & & \\
\hline
\end{tabular}


Appendix 2: Physical characterization for MASS WAF (left), CEWAF WSF (middle) and HEWAF WSF (right). EpiFluorescence microscope images showing no fluorescing entities (top) and EEMs of the preparations showing signals for the soluble components only at $\lambda \mathrm{ex}=280 \mathrm{~nm} / \lambda \mathrm{em}=315 \mathrm{~nm}$ (bottom)
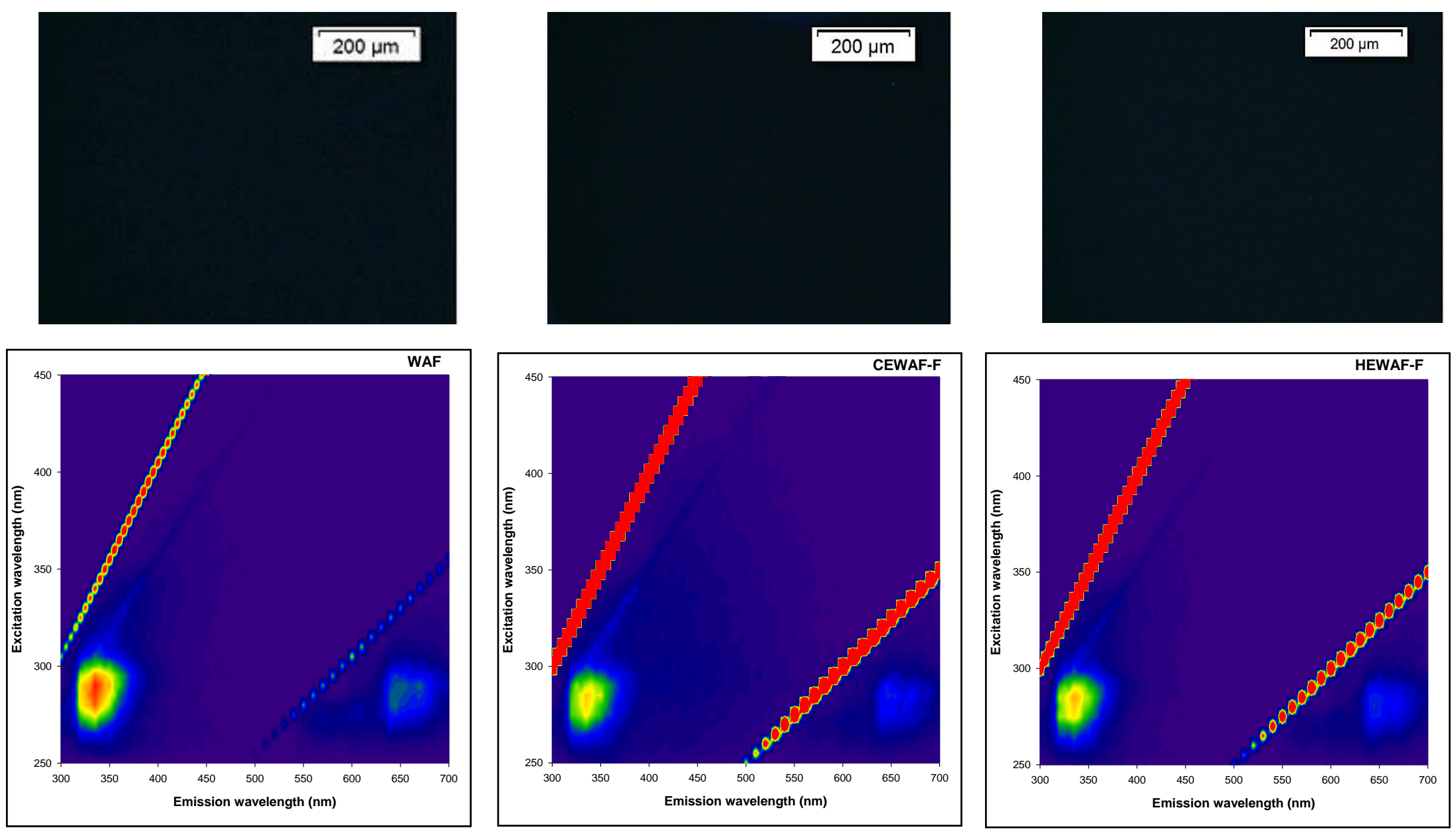
Appendix 3: Concentrations of the listed PAHs for the natural source and alkane oil

\begin{tabular}{lccc}
\hline Compound & $\begin{array}{c}\text { Concentration in } \\
\text { natural source oil } \\
(\mathrm{mg} / \mathrm{L})\end{array}$ & $\begin{array}{c}\text { Nominal } \\
\text { concentration in } \\
\text { alkane oil (mg/L) }\end{array}$ & $\begin{array}{c}\text { Measured } \\
\text { concentration in } \\
\text { alkane oil (mg/L) }\end{array}$ \\
\hline Naphthalene(N0) & 1462 & 1470 & 1515 \\
C1-Naphthalene(N1) & 1732 & 1710 & 1671 \\
C2-Naphthalene(N2) & 1215 & 1197 & 1185 \\
Fluorene(F0) & 123 & 121 & 121 \\
Phenanthrene(P0) & 613 & 612 & 672 \\
Dibenzothiophene(DBT0) & 46.7 & 47.4 & 56.7 \\
C1-Dibenzothiophene(DBT1) & 142 & 146 & 141 \\
C2-Dibenzothiophene(DBT2) & 201 & 208 & 207 \\
Pyrene(PY) & 12.8 & 11.7 & 13.5 \\
Chrysene(C0) & 43.9 & 44.3 & 44.1 \\
\hline
\end{tabular}

